\title{
Distance Estimation for 3D Freehand Ultrasound-Scans with Visualization System
}

\author{
by
}

Hsi-Cheng Don Wei

A thesis submitted to the Faculty of Graduate and Postdoctoral Affairs in partial fulfillment of the requirements for the degree of

\section{Master of Applied Science}

in

Biomedical Engineering

Department of Systems and Computer Engineering Carleton University

Ottawa, Ontario

(C) 2017, Hsi-Cheng Don Wei 


\section{Abstract}

Some primary concerns with freehand 3D ultrasound are time-consuming calibration operations, overall accuracy, and computation time during the $3 \mathrm{D}$ reconstruction process. A procedure was developed to reduce the calibration time to only 10 seconds by planning the path of the probe. A decrease in marginal error may improve the overall accuracy by searching axial and lateral distances with a maximum correlation coefficient, and was based on constructing an adaptive decorrelation curve from images containing Rayleigh and coherent scatters. Marginal errors were reduced in the range of $3 \%$ to $11 \%$ when the distance estimation was between $0.15 \mathrm{~mm}$ and $0.375 \mathrm{~mm}$ in in-vitro studies. To save computation time within the $3 \mathrm{D}$ reconstruction, a low-resolution $3 \mathrm{D}$ reconstruction algorithm is proposed so that verification can be completed prior to computing a high-resolution reconstruction. 


\section{Acknowledgements}

I would like to thank my thesis supervisor Dr. Chris Joslin, School of Information Technology, Carleton University, for all the help, friendly guidance, and inspiration. I would also like to thank Dr. WonSook Lee, School of Electrical Engineering and Computer Science, University of Ottawa, for granting me the use of her equipment so the experiments could be completed. I would like to thank Hamed Mozaffari and Zhen Qu for their help in using the equipment and setting up phantoms as well. I am grateful to my lab mates, friends, and family for their support and encouragement so my Master's degree can be completed. 


\section{Table of Contents}

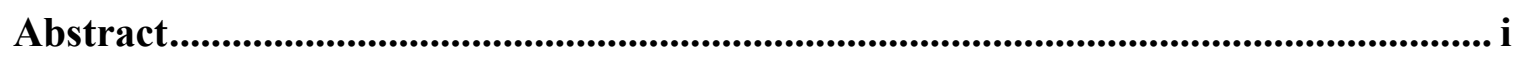

Table of Contents ...........................................................................................................ii

List of Tables .................................................................................................................... vii

List of Acronyms ............................................................................................................................. xii

Chapter 1: Introduction ..................................................................................................... 1

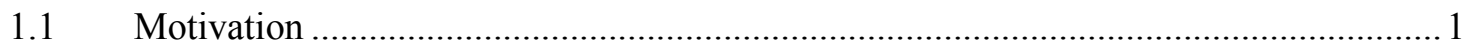

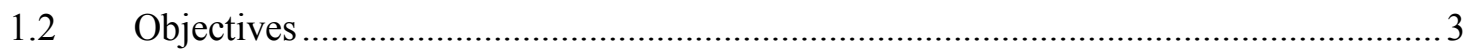

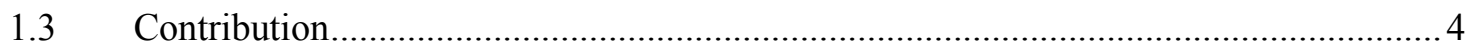

Chapter 2: Background ................................................................................................. 6

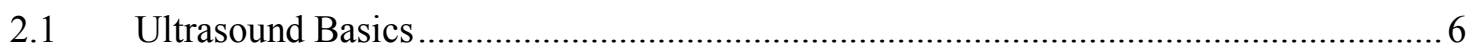

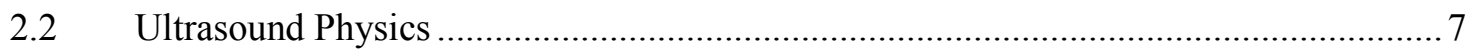

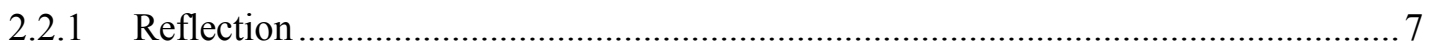

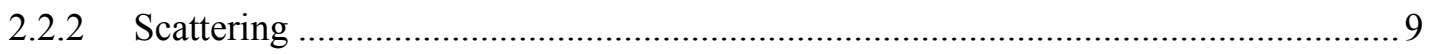

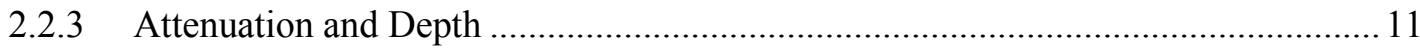

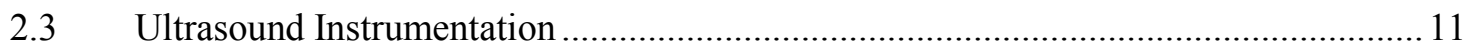

2.4 Ultrasound Image Modality/Modes .......................................................................... 13

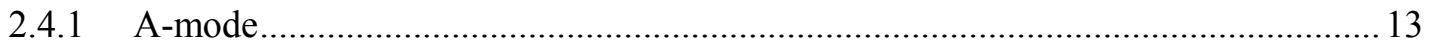

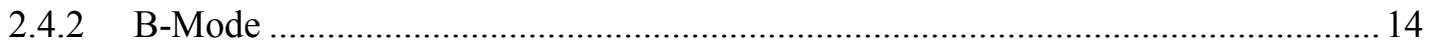

2.5 Usage in Ultrasound Musculoskeletal Imaging ........................................................ 14

2.6 Limitations of the 2D Ultrasound System …………………………………….....15

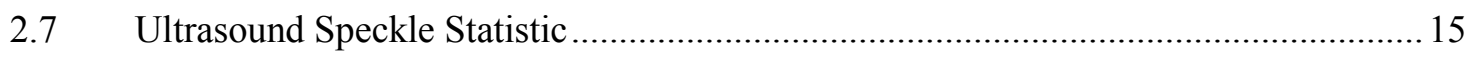

2.7.1 Mathematical Model of Speckles .......................................................................... 16

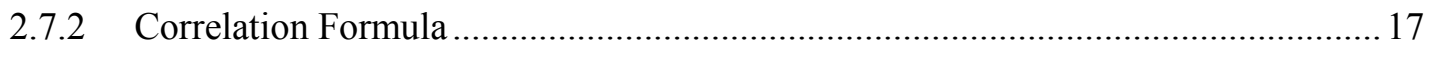

2.7.3 Speckle for Out-of-Plane Motion Estimation......................................................... 19 
Chapter 3: State of Art ........................................................................................................... 20

3.1 Acquisition Techniques for 3D Ultrasound System ............................................20

3.1.1 Acquisition Technique from Mechanical Sweep Probe .........................................220

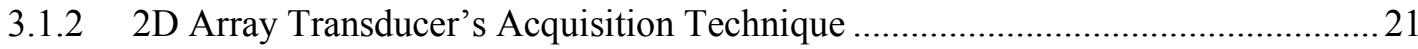

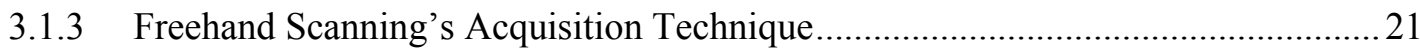

3.1.3.1 Freehand Scanning's Acquisition Technique with Tracking Device ..............22

3.1.3.2 Freehand Scanning's Acquisition Technique without Tracking Device ..........23

3.2 Components of the 3D Freehand Ultrasound System...............................................23

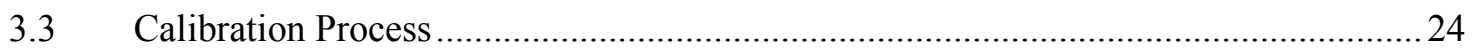

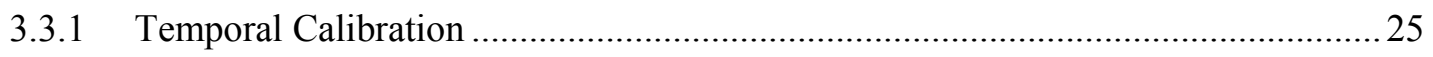

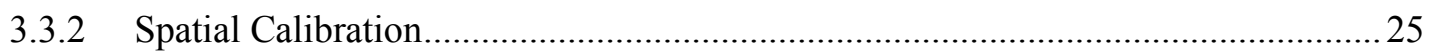

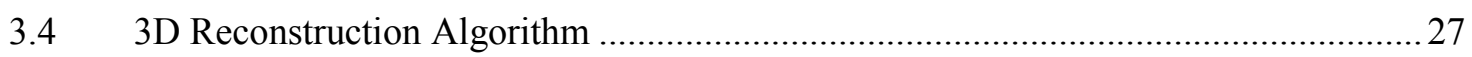

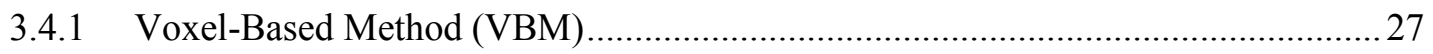

3.4.2 Voxel-Based Method with Interpolation (VBMI) ..............................................28

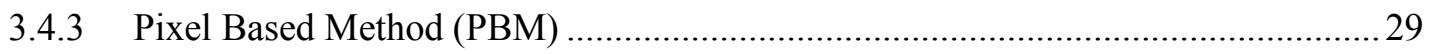

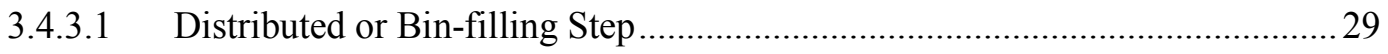

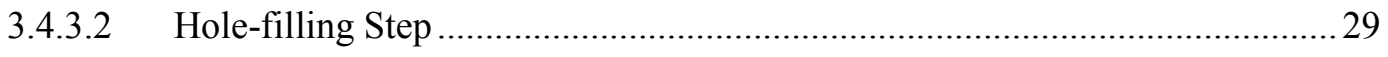

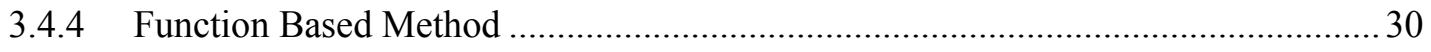

3.4.4.1 Position Sensor Accuracy in 3D Reconstruction........................................... 31

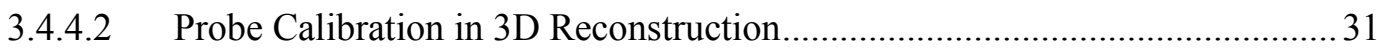

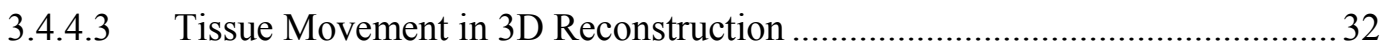

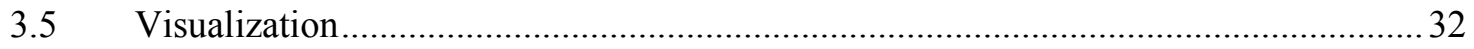

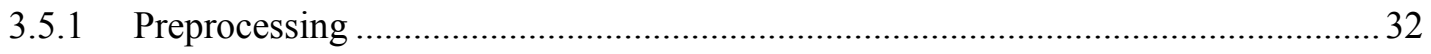

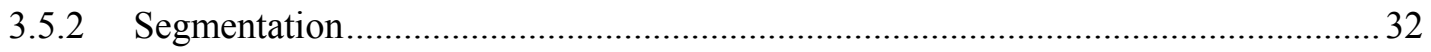

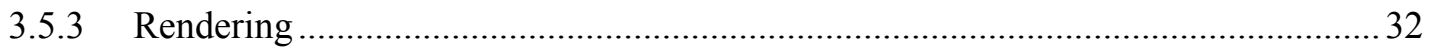

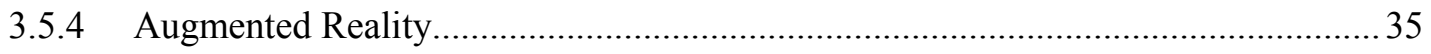




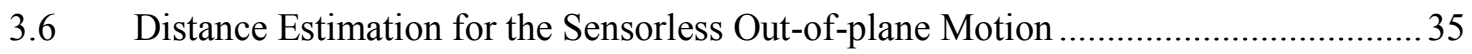

3.6.1 Speckle with In-plane Motion Distance Estimation .............................................. 35

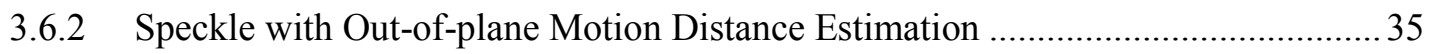

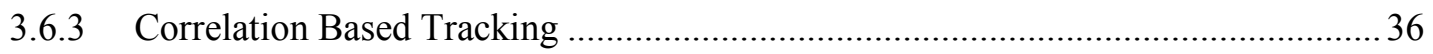

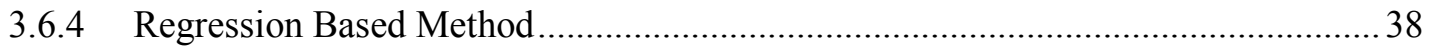

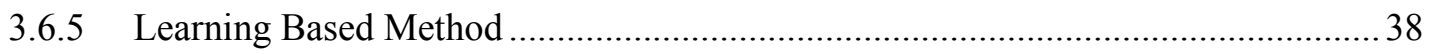

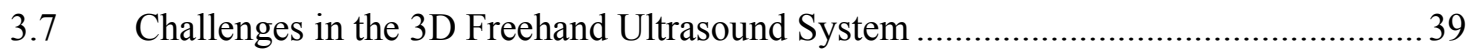

Chapter 4: Methodology............................................................................................................ 40

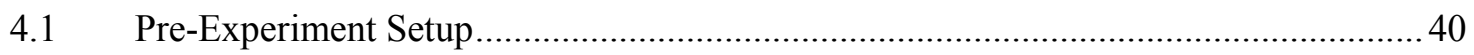

4.2 Experimental Setup for Medical Ultrasound Image System and the Probe ................ 42

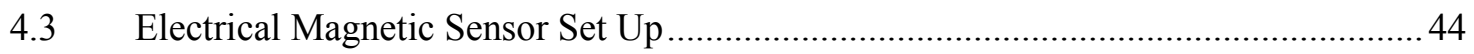

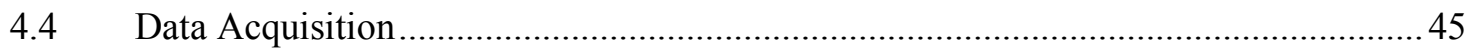

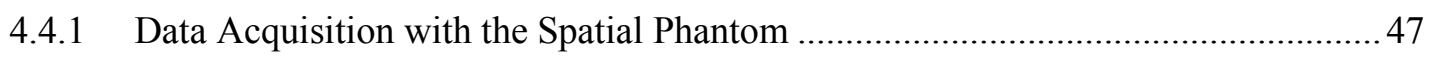

4.4.2 Data Acquisition with the Speckle Phantom...................................................... 48

4.4.3 Data Acquisition with the Meat Phantoms......................................................... 48

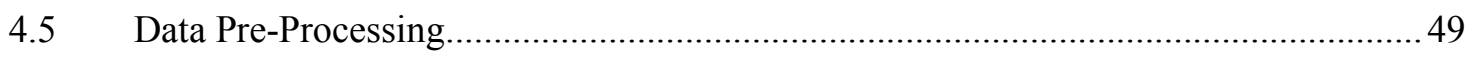

4.5.1 Data Preprocessing in Temporal Calibration .................................................... 50

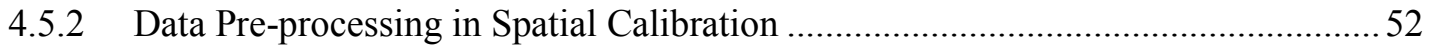

Chapter 5: Data Analysis and Results ...................................................................... 62

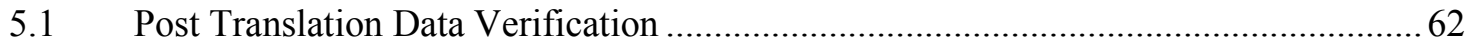

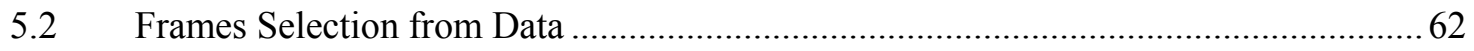

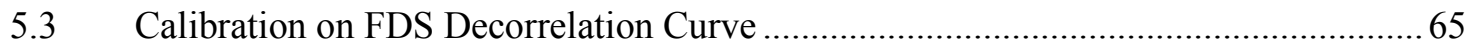

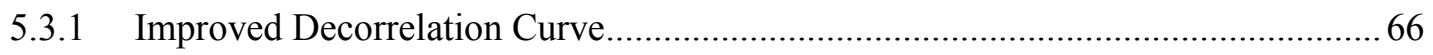

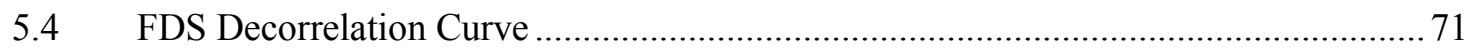

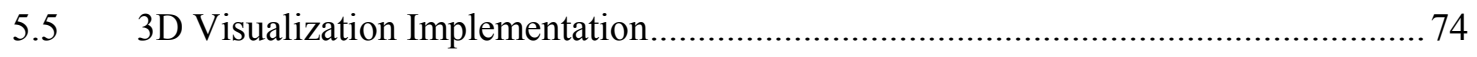

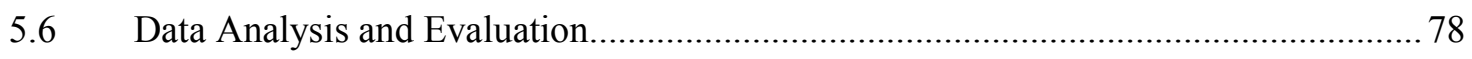




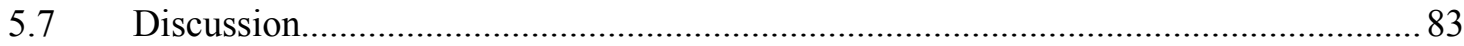

Chapter 6: Conclusion and Future Research ..................................................................... 87

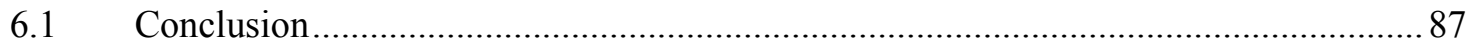

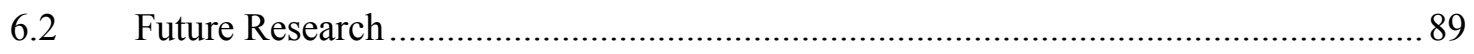

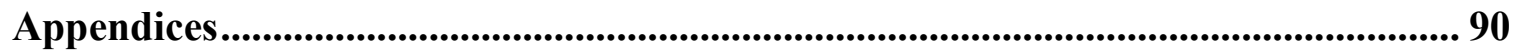

Appendix A : External Library and System Environment............................................... 90

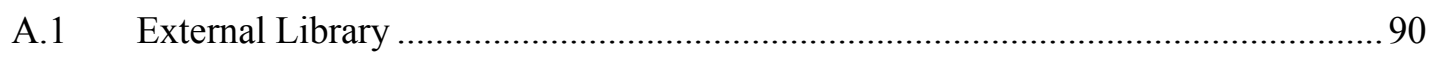

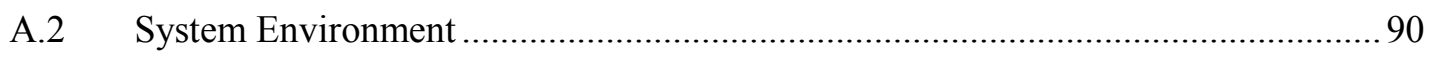

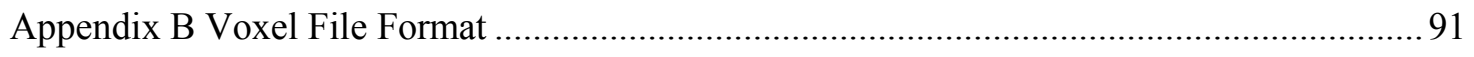

Appendix C Procedures for making the Spatial Phantom .................................................. 92

Appendix D Procedures for making the Speckle Phantom.................................................. 92

Appendix E Procedures for making the Meat Phantoms ..................................................... 93

Appendix F Procedures for Setting up SonixTablet Ultrasound System................................ 93

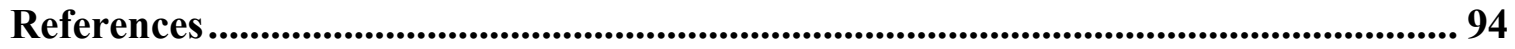




\section{List of Tables}

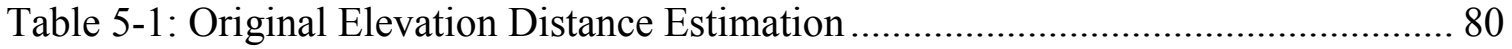

Table 5-2: Improved Elevation Distance Estimation.................................................. 80

Table 5-3: Percentage change in chicken and beef phantoms for standard deviation ...... 83

Table 5-4: Computation time for the chicken/beef data set....................................... 85

Table 5-5: Comparisons between different reconstruction algorithms .......................... 86 


\section{List of Figures}

Figure 1-1: Frontal plane view of hip joint in for FAI from [7]

Figure 2-1: Axial, elevation, and lateral axes for sweeping ultrasound transducers. The axial direction represents the direction of the beam. The lateral direction is perpendicular to the direction of the beam. The elevation direction is parallel to the sweeping direction in in-plane motion and perpendicular to other axes. Reconstructed from [23] ................. 6 Figure 2-2: Interactions between ultrasounds and tissues lead to (a)Reflection (b)Scattering (c)Diffuse Reflection from [28] ........................................................ 8

Figure 2-3:Example of grainy appearance in the human thigh tissue of Ultrasound B-

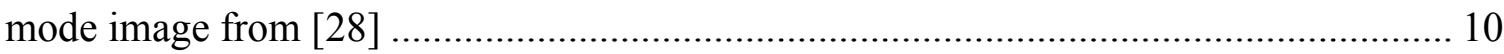

Figure 2-4: Various components in the basic ultrasound probe from [25] ...................... 12

Figure 2-5: Detection of the envelope in A-mode ultrasound image from [30] .............. 13

Figure 3-1: Various acquisition techniques for 3D US image system(a) Mechanical scan with 1D array transducer(b) 2D array scan(c) Freehand scan from [9] .......................... 21

Figure 3-2: Coordinates for the EM trackers from [52] .............................................. 22

Figure 3-3: Various coordinate systems including image plane (P), Receiver (R), Transmitter (T) and Volume Reconstruction (C) from [58] ........................................ 26

Figure 3-4: Visualization pipeline. Reconstructed from [82] ......................................... 33

Figure 3-5: Procedures for the volume ray-casting from [86] ......................................... 34

Figure 3-6: Raytracing with two light sources. Reconstructed from [88] ........................ 34

Figure 3-7: Concept of the elevation speckle decorrelation curve from [17] ................... 36

Figure 3-8: Imperfect elevation focus of the ultrasound probe from [17] ...................... 37

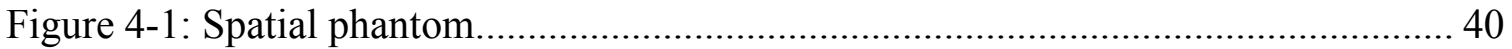


Figure 4-2: Speckle phantom (left). The Raleigh scattering simulated from graphite powders in B-scan of Speckle phantom (right).

Figure 4-3: The size of chicken, beef and eraser in meat phantom for the elevation distance estimation 42

Figure 4-4: Ultrasonix SonixTablet ultrasound image system 43

Figure 4-5: Sensor is attached on the ultrasound probe bound with electrical tape to detect EM field 44

Figure 4-6: The EM source (left) and system electronic unit (right) in the Patriot sensor for producing the EM field 45

Figure 4-7: An overview of data acquisition and data analysis for the elevation distance estimation reconstructed from [28] 46

Figure 4-8: Cropping the image in the beef phantom

Figure 4-9: Temporal calibration in beef phantom in 3D from raw data from EM sensor attached to the probe 51 Figure 4-10: Temporal calibration in beef phantom in the direction of $\mathrm{Z}$ axis with respect to frame from raw data 51

Figure 4-11:Synchronization of the Patriot sensor with the ultrasound imaging system at the same frame rate from [28]. 52 Figure 4-12: Different coordinate systems for image plane (P), receiver/sensor (R),

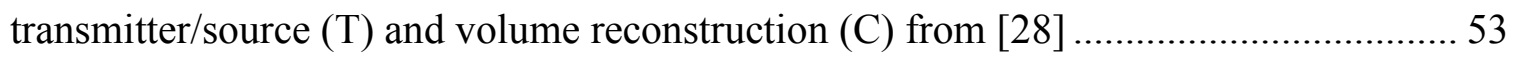
Figure 4-13: Euler angles in Z-Y-X for the Patriot sensor from [99] ........................... 55 
Figure 4-14: Normal distribution for values in X-axis at the center location of volume reconstruction from various images in different directions. Variance is $6.3813 \mathrm{~mm}^{2}$.

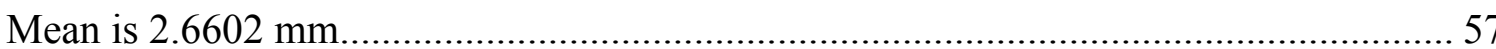

Figure 4-15: Normal distribution for values in $\mathrm{Y}$ axis at the center location of volume reconstruction from various images in different directions. Variance is $2.8611 \mathrm{~mm}^{2}$.

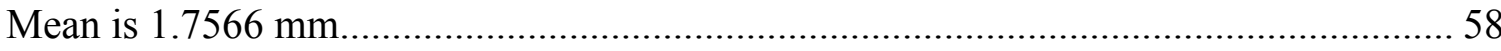

Figure 4-16: Normal distribution for values in $\mathrm{Z}$ axis at the center location of volume reconstruction from various images in different directions. Variance is $0.0122 \mathrm{~mm}^{2}$.

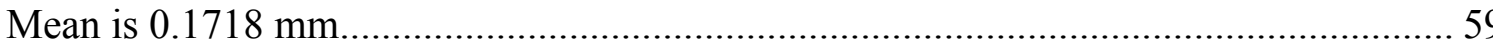

Figure 5-1: 3D coordinates for Beef phantom in post-translation $[\mathrm{cm}] \ldots \ldots \ldots \ldots \ldots \ldots \ldots \ldots \ldots . . . . \ldots 3$

Figure 5-2: 3D coordinates for Chicken phantom in post-translation $[\mathrm{cm}] \ldots \ldots \ldots \ldots \ldots \ldots . . . . . . . .63$

Figure 5-3: Dividing an image into 96 patches for B-scan in speckle phantom............... 64

Figure 5-4: Axial decorrelation curve of speckle phantom for patch 5 and patch 13 in the first row. Patch 6 and patch 14 are in the second row. 68

Figure 5-5: Lateral decorrelation curve of speckle phantom for patch 5 and patch 13 in the first row. Patch 6 and patch 14 are in the second row. 69 Figure 5-6: Elevation decorrelation curve of speckle phantom for patch 5 and patch 13 in the first row. Patch 6 and patch 14 are in the second row. 70 Figure 5-7: FDS adaptive decorrelation curve in the original elevation distance estimation

Figure 5-8: FDS adaptive decorrelation curve in the improved elevation distance estimation 74 
Figure 5-9: An improved algorithm for calculating voxel position and its intensity in

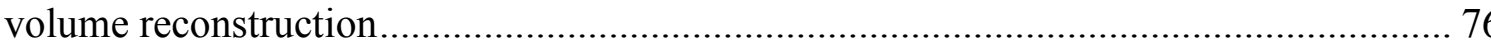

Figure 5-10: Low-resolution algorithm for displaying the 3D structure of the targeted anatomy at a particular frame 77

Figure 5-11: Low-resolution algorithm for displaying the 3D structure of the targeted anatomy in series 77

Figure 5-12: Relocation of ROI in Beef phantom at frame 317 ................................. 78

Figure 5-13: Relocation of ROI in Chicken phantom at frame 296 .............................. 79

Figure 5-14: Original elevation distance estimation on Beef phantom ......................... 81

Figure 5-15: Improved elevation distance estimation on Beef phantom ....................... 81

Figure 5-16: Original elevation distance estimation on chicken phantom ..................... 82

Figure 5-17: Improved elevation distance estimation on chicken phantom ................... 82

Figure 5-18: Systematic error for large or small distance in the elevation direction from $[28]$ 


\section{List of Acronyms}

2D Two-Dimensional

3D Three-Dimensional

CPU Central Processor Unit

CT Computerized Tomography

DOF Degree Of Freedom

DS Distributed Step

FAI Femoro-Acetabular Impingement

FBM Function-Based Method

FDS Fully Developed Speckle

GPU Graphical Processor Unit

HFS Hole filling Step

MRI Magnetic Resonance Image

MSDS Material Safety Data Sheet

PBM Pixel Based Method

PNN Pixel Nearest Neighbor

RF Radio Frequency

ROI Region of Interest

US Ultrasound

VBM Voxel-Based Method

VBMI Voxel-Based Method Interpolation

VNN Voxel Nearest Neighbor 


\section{Chapter 1: Introduction}

\subsection{Motivation}

From Wackerhage et al. [1], musculoskeletal mass accounts for about 50 percent of the mass of the average human body. Though there are a plethora of musculoskeletal diseases spanning from the tendinitis to arthritis, the main motivation for this research is to help in the diagnosis and treatment of Femoro-Acetabular Impingement (FAI), a musculoskeletal abnormality.

From Leunig et al. [2] mechanical factors for FAI may include one or all of the following: bone deformations of either the femoral head or the acetabulum, and spatial mal-orientations of the femoral head with respect to the acetabulum. These factors can result in cartilage tears within the hip joint on either an articular cartilage or a labral cartilage in Figure 1-1, and consequently result in abnormal bone contact, further deteriorating the hip joint.

Tan et al. [3] infer musculoskeletal images have advanced rapidly due to significant developments in image modalities including Magnetic Resonance Imaging (MRI), Computed Tomography (CT) and ultrasound (US). From Lerck et al. [4] these image modalities are applied tos diagnose FAI by producing high-quality images around the hip area finally. However, a primary concern surrounding CT is ionizing radiation, which may be unsafe for everyone especially high-risk patients including pregnant women.

Compared to CT, US and MRI do not use ionizing radiation. US is less timeconsuming and cheaper than MRI. From Nelson et al. [5] the US is becoming widely adopted and is considered the least invasive, but its image resolution is lower than CT or 
MRI. According to Wakefield et al. [6], The US imaging is applicable in diagnosing joint scans, due to the small size of the US probe which permits easier rotation and can be more easily oriented into the region of interest(ROI). The ultrasound system also provides a real-time scanning feature which is cheaper than the CT and MRI. Due to the lowest cost, shortest wait time and lowest health risk in patients among other image modalities, US becomes a popular research area in diagnosing FAI.

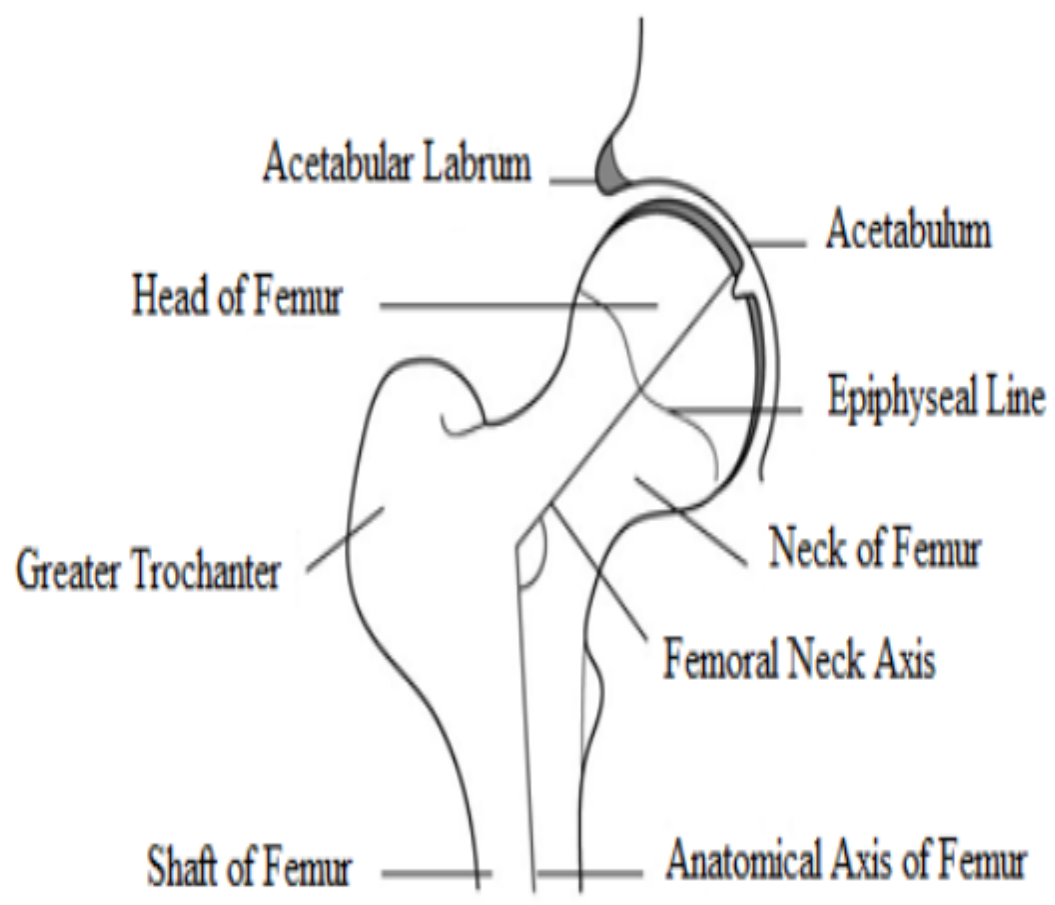

Figure 1-1: Frontal plane view of hip joint in for FAI from [7]

To overcome limitations of the 2D ultrasound system such as subjective results due to sonographers' varying degrees of experience from integrating images mentally, Fenster et al. [8] mention the 3D ultrasound imaging system has been developed. The 3D ultrasound imaging systems reduce the dependency on the sonographers' interpretive scanning experience and provide a wider viewing range for ROI.

The freehand ultrasound system or scan which reconstructs $3 \mathrm{D}$ anatomical structures from an unidimensional array transducer is preferred than the 2D array scan and mechanical scan according to Karadayi et al. [9] due to its low cost, simplicity, and 
flexibility. The freehand ultrasound increases diagnostic precision and performance as the probe can scan through anatomical structures in any arbitrary direction including parallel/perpendicular to any of three axes, as positioning and orientation are recorded simultaneously through electrical magnetic (EM) sensors or optical sensors attached to the probe.

In support, Gee et al. [10] mention that the volume of the anatomical structures in ROI can be formed by obtaining the corresponding positions of the three axes in 3D space. Even though the US currently has a low image quality, freehand 3D ultrasound presents itself as an irresistible topic of research in diagnosing FAI. Despite the aforementioned advantages that come with the use of 3D freehand ultrasound scans with visualization systems, there are several challenges that should be evaluated.

- Rapid and accurate calibrations when attaching trackers on the probe are required to maintain data consistency and reduced wait time for sonographers for the future clinical application since properties of phantom including elasticity degrades over time.

- Internal surface move including fat/muscle deformation and external surfaces move including skin deformation during the data acquisition make accuracy a major concern for elevation distance estimations.

- 3D reconstructions of targeted anatomical structures need to be derived from $2 \mathrm{D}$ images in a timely manner so the real-time visualization feedback is provided during the data acquisition.

The ultimate goal for the 3D freehand ultrasound system is to allow the probe to scan in any direction without trackers which require no additional calibration procedures while reconstructing the targeted anatomical structure in real-time. This would allow immediate feedback including the positions and $3 \mathrm{D}$ anatomical structures to be provided to the sonographer.

\subsection{Objectives}

The desired outcome of the 3D freehand ultrasound system is to have the accurate elevation distance estimation and a quick spatial calibration procedure with low 
computation time during the volume reconstruction. Three objectives need to be addressed to achieve the desired outcome.

The first objective involves the spatial calibration a currently time-consuming step, and can last two minutes from Hsu et al. [11], [12] so sonographers are required to wait during the data acquisition process. At the same time, Bude et al. [13] infer data consistency which is needed to mimic tissue properties of the in-vitro study begins to decrease in phantoms from Kim et al. [14] due to losing their desired properties over time. Thus, the acquisition is subjective to give different result due to these changes. When an EM sensor is attached to the ultrasound probe by detecting the strength of magnetic fields from the source, global motion data is collected and displayed through the freehand ultrasound system. The sonographer is required to precisely calibrate the data between the external sensor attached to the probe and images captured in ultrasound imaging machine and vice versa.

The second objective involves the high marginal errors during the elevation distance estimation in the 3D freehand ultrasound system. From Gee et al. and Gao et al. [15], [16] Cambridge heuristic method for the elevation distance estimation considers both the effects of Rayleigh and coherent scatters. However, there are large standard deviations or marginal errors in their studies, making the results too variable. From Hajian-Tilaki et al. [17] lower marginal errors make clinical studies more precise.

The third objective involves the low-resolution voxel during the volume reconstruction for the visualization system in the 3D freehand ultrasound system. From Solberg et al. [18] the computations in volume reconstruction can last several hours so low-resolution voxel grids can be displayed before presenting high-resolution voxel grids. According to Sanches and Marques et al. [19], the verification process may be completed at an earlier stage.

\subsection{Contribution}

The first contribution is to reduce the data acquisition time for spatial calibration, this thesis offers a procedure based on placing the probe on the spatial phantom on a designated path during spatial calibration, allowing the data acquisition time for the spatial calibration to be reduced to 10 seconds. 
The second contribution is to reduce marginal errors during the elevation distance estimation. The method posited here determines the maximum correlation coefficients between the current frame and the next frame of the image in the speckle phantom within both axial and lateral directions. From Gee et al. [16] speckle phantoms are important to simulating Rayleigh scatters in the real tissue. From Tuthill et al. [20] correlation coefficients are calculated between frames in the B-mode ultrasound images in the speckle phantom. Standard deviations of measured distances are reduced, which improves accuracy in elevation distance estimations.

The third contribution is to decrease the reconstruction computation time, this thesis posits a simpler algorithm that averages pixels between frames to determine the voxel value. Consequently, low-resolution targeted anatomical structures can be displayed in the $3 \mathrm{D}$ space earlier. 


\section{Chapter 2: Background}

\subsection{Ultrasound Basics}

From Silver [21] ultrasound is defined as acoustic waves with frequencies beyond 20 $\mathrm{kHz}$. These waves are transmitted as pulses through an electrical medium to a mechanical one comprised of acoustical transducers. Certain portions of the waves are reflected after hitting boundary layers such as skin. After the reflected waves are received by the transducer again, they are processed to create images.

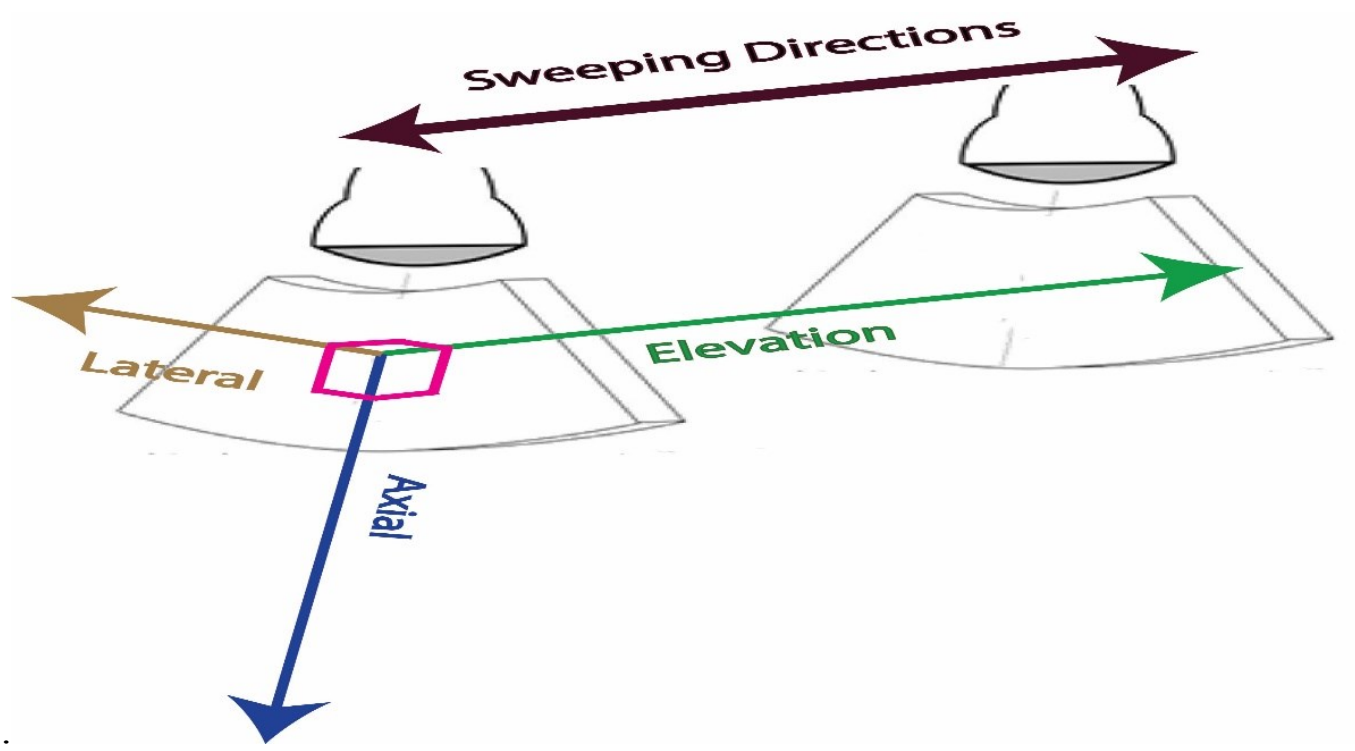

Figure 2-1: Axial, elevation, and lateral axes for sweeping ultrasound transducers. The axial direction represents the direction of the beam. The lateral direction is perpendicular to the direction of the beam. The elevation direction is parallel to the sweeping direction in in-plane motion and perpendicular to other axes. Reconstructed from [22] 
One of methods to acquire ultrasound images is called freehand-scan. From Karadayi et al. [9] sonographers are required to sweep the ROI with a 1D transducer inside a probe on the target surface. Subsequently, a series of US B-mode images are constructed while recording the images' positions and orientations simultaneously. From Silver [21] medical ultrasound devices have a frequency range from $1 \mathrm{MHz}$ to $20 \mathrm{MHz}$ due to a need to accommodate different image resolutions and penetration depths in the clinical settings.

Within an ultrasound system, from Szabo [23] the axial direction (the perpendicularity between the ultrasound beam and direction of sound propagation from the transducer to an anatomical structure such as skin); the lateral direction (the vertical plane with respect to the ultrasound beam) and the elevation direction (the parallelity in the axis of sweeping and perpendicularity with other axes) as displayed in Figure 2-1.

\subsection{Ultrasound Physics}

From Silver [21] when ultrasounds transmit the longitudinal waves into physical media, the direction of sound propagation and displacement are the same. From Hoskins [24] the speed of propagation is dependent on the type of medium in which it travels through. In addition, from Silver [21] the interaction between sound waves and real tissues affects the outcome of ultrasound images as illustrated in Figure 2-2. The reflections, scatterings, attenuations, and depths listed below describe the physical properties of interactions between the ultrasounds and tissues.

\subsubsection{Reflection}

Silver [21] explains that ultrasound images are formed when reflective waves (or echoes) return to the transducers after reflecting off of tissue boundaries. The amount of reflection is dependent upon the change in acoustic impedance between tissue types.

As illustrated in Equation 2.1, acoustic impedance (z) is the product of sound (c) and density $(\rho)$. From Szabo [23] it measures the amount of transmission and reflection of the ultrasound pulse at a boundary.

$$
z=\rho c
$$


The amount of ultrasound pulse reflected $(\mathrm{R})$ or transmitted $(\mathrm{T})$ while propagating through different biological interfaces is determined by Equation 2.2 or 2.3. Equations 2.2 and 2.3 are best known as the intensity reflection and intensity transmitted coefficients. The $Z_{1}$ and $Z_{2}$ represent impedances in the first and second media respectively. From these equations, Bushong [25] explains that the amount of transmissions or reflections of ultrasound pulses is dependent on the acoustic impedance between two media. If the impedance between them is large, the pulse will be reflected more and transmitted less.

$$
\begin{aligned}
& R=\left(\frac{Z_{2}-Z_{1}}{Z_{2}+Z_{1}}\right)^{2} \times 100 \\
& T=100-R
\end{aligned}
$$

Equation 2.2 assumes a smooth and flat boundary interface, but Kremkau and Frederick [26] caution that reflections from uneven surfaces need to be accounted for.

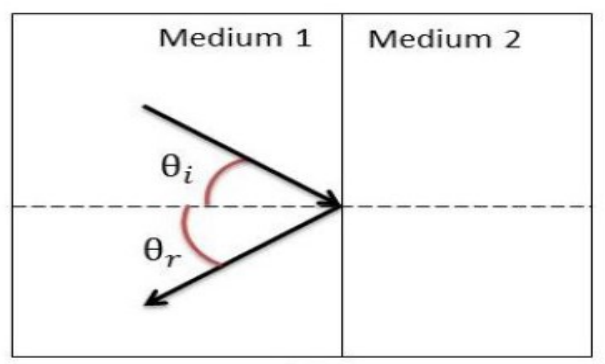

(a)

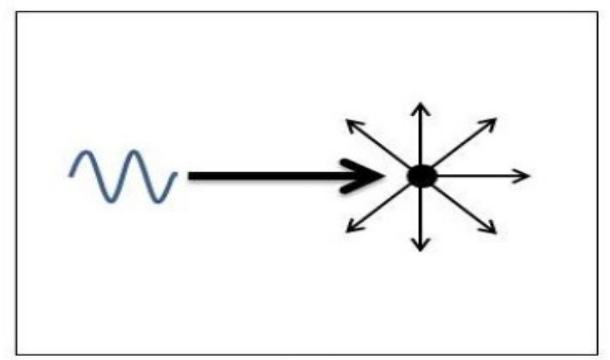

(b)

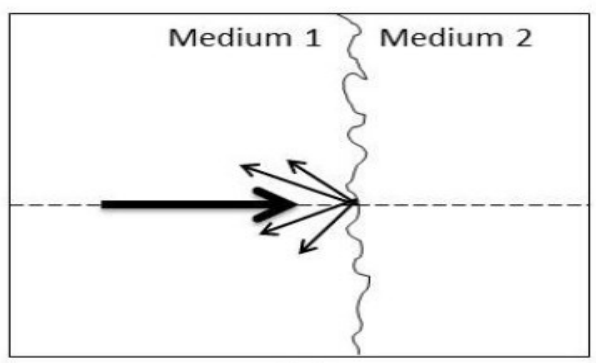

(c)

Figure 2-2: Interactions between ultrasounds and tissues lead to (a)Reflection (b)Scattering (c)Diffuse Reflection from [27]

Silver [21] infers that there are both specular and diffuse reflections according to the properties of the tissue boundary. As shown in Figure 2-2a, specular reflection occurs when there is a great acoustic impedance change due to a large, flat, and smooth 
boundary. As shown in Figure 2-2c, diffuse reflection occurs when there are many reflections coming from various angles in a rough or uneven boundary.

\subsubsection{Scattering}

Scattering occurs when the target's size is much smaller than the ultrasound's wavelength. Consequently, the general law of reflection may not be valid here as waves are scattered in the unchanged directions monotonically. The pattern of scatterings as displayed in Figure 2-2b is referred to as non-specular reflection.

Bahramabadi [27] details that scatterings play an important role in the formation of ultrasound images of the skeletal muscular system. Muscle fibers are fundamental building units in tissue formation and are oriented in different directions. When the ultrasound probe scans where the ultrasound beam runs parallel to the muscle fibers in the axial direction, tissues cause the ultrasound waves to move non-monotonically or in all directions. As such, Hoskins et al. [24] describe how muscles displayed in an image will vary each time due to differing orientations of the ultrasound probe and directions of the tissue fibers.

From Szabo [23] tissues are inhomogeneous media since they contain many layers of structure and their movements with various length scales, thus cannot be uniform across all sections. Silver [21] mentions that acoustic speckles occur when ultrasound pulses travel through the tissues and encounter smaller particles than that of the ultrasound wavelength, which creates a simultaneous formation of several echoes at the end of the transducers resulting in either a constructive or a destructive interference, also known as emphasis or debilitation. These small particles lead to scattering of the ultrasonic waves into various directions and are also known as scatters.

When the ultrasound beam travels through the tissue, the final echoes switch between the constructive and destructive interference, which causes the dot patterning, or grainy appearance, to be displayed on the image as in Figure 2-3. The grainy quality represents interference patterns distributed randomly in the scatter scans. This concept is known as acoustic speckle and is considered as one of the acoustic noises in ultrasound images. The scattering interference pattern is applied for the elevation distance estimation by measuring the correlation difference between frames. 


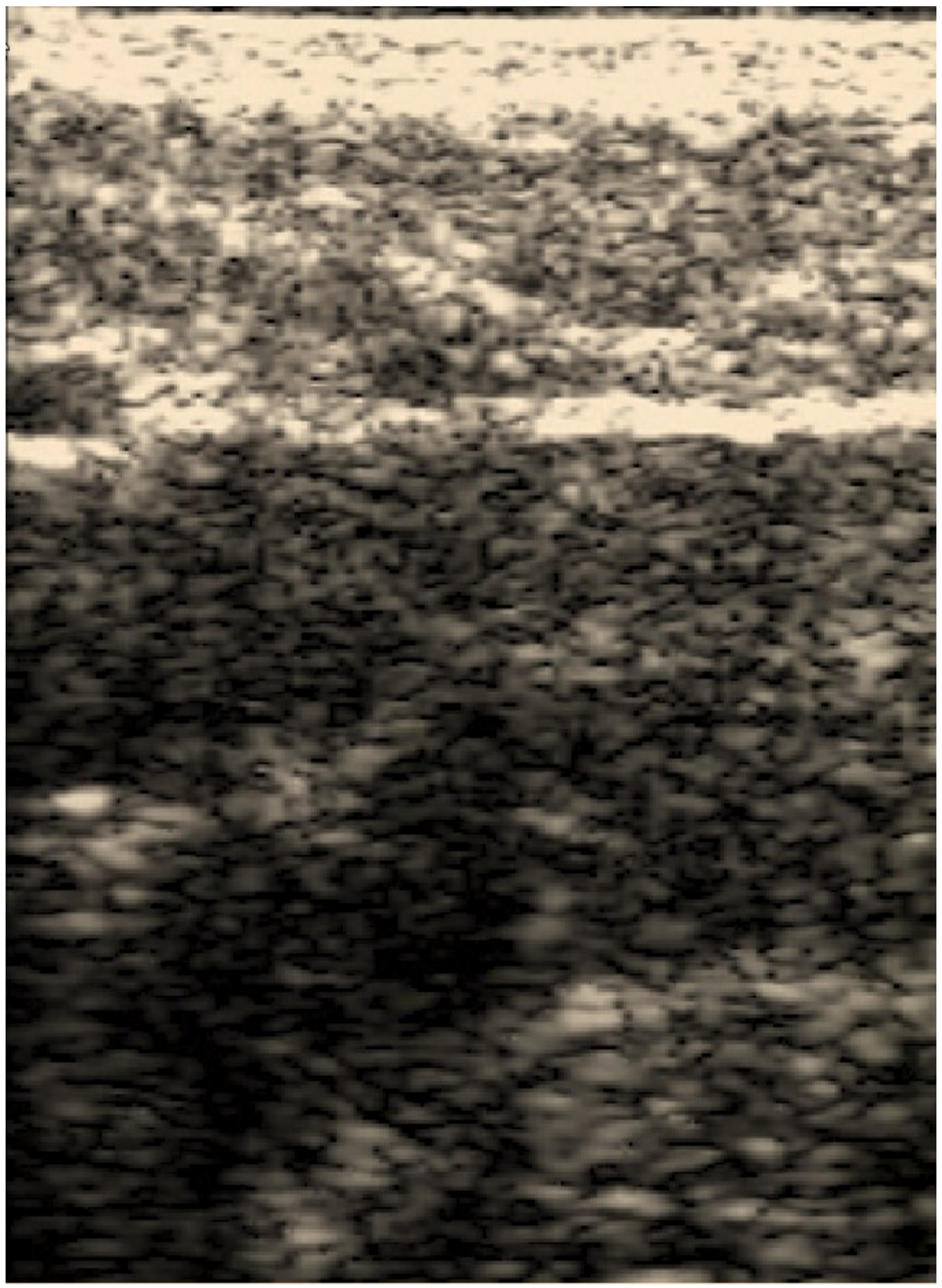

Figure 2-3:Example of grainy appearance in the human thigh tissue of Ultrasound B-mode image from $[27]$ 


\subsubsection{Attenuation and Depth}

From Hedrick et al. [28], an attenuation occurs when the ultrasound wave propagates through anatomical structures, some of its energy is transformed from the sound energy to heat energy via absorption. Hoskins [24] notes that the farther the ultrasound waves travel in space, the more energy will be absorbed. In addition, attenuation is an important factor with respect to the penetration depth in which the ultrasound wave travels. The deeper the ultrasound wave travels, the more attenuation there will be. It is necessary to compensate for this effect by adjusting the attenuation effect from the diagnostic equipment.

Szabo [23] infers that by knowing the speed of sound in the tissue and the time intervals $(\Delta \mathrm{T})$ between the transmitted and reflected round trip echoes from the transducer, the depth can be calculated as shown in Equation 2.4. Dividing by two allows one to account for the wave round trip. The velocity of tissue is $1540 \mathrm{~m} / \mathrm{s}$ as labeled in symbol c.

$$
d=\frac{c \cdot \Delta T}{2}
$$

\subsection{Ultrasound Instrumentation}

Silver and Prince et al. [21], [29] indicate that the ultrasound probe is comprised of transducer elements, which consist of piezoelectric material for converting electrical transmission pulses into ultrasonic pulses (or vice versa). Electrical signals are applied to the transducer materials to cause either expansion or contraction. A pressure wave is subsequently generated and transmitted to an anatomical structure. An electrical signal generated by the collision of the reflected acoustic wave at the transducer end, which records its pattern.

A basic transducer consists of a narrow rectangular piezoelectric material, a matching layer, and a backing layer surrounded by a case as depicted in Figure 2-4. Silver and Bahramabadi [21], [27] explain that when unwanted sound waves such as backward traveling waves or reflected echoes are received from a transducer in the probe and tissue interface, the backward layer removes those unwanted echoes. 


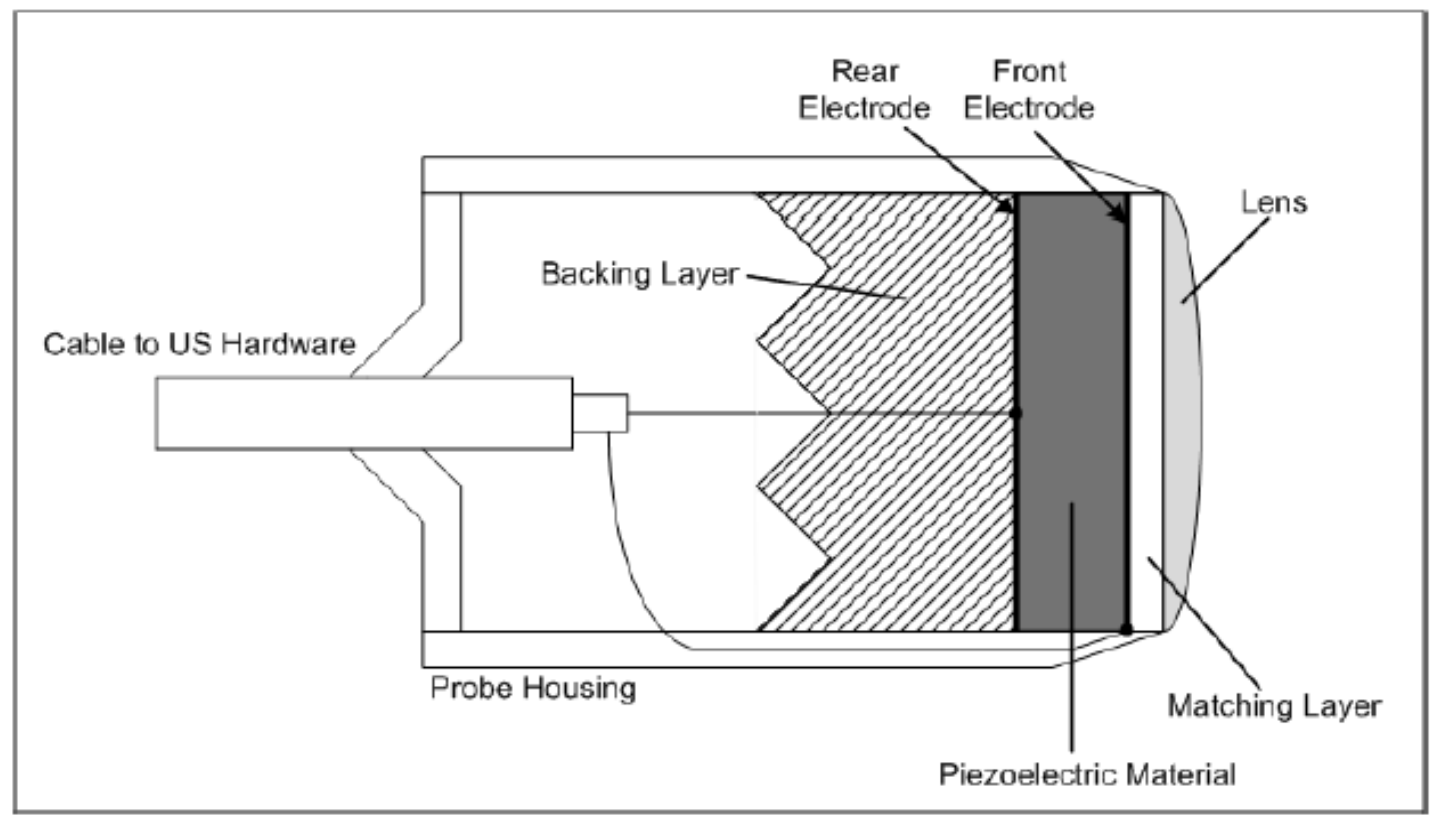

Figure 2-4: Various components in the basic ultrasound probe from [24]

From Hedrick et al. [28], a matching layer effectively reduces this impedance difference between the piezoelectric material and real tissue when the reflected accusative waves return from matching layer to backing layer via PZT.

Generally, there are various types of probes including linear, curvilinear and phase arrays, which differ based on the arrangements found in the transducers of the probe. In this case, the keyword transducer specifically refers to the piezoelectric material. In this thesis, a linear array probe is used since it is most affordable.

There is a linear array of narrow rectangular transducers with a dimension of $1 \mathrm{xn}$ elements within the piezoelectric material. The individual element inside the linear transducer produces a series of waves of small ultrasound beams. There is a delay required for receiving the reflected ultrasound pulse by the transducers before the next series of transducers fire. By having different groups of transducers firing in series, the sound beam spans from one transducer to the next across all transducers in the array. Therefore, each single element $1 \times 1$ in the array of the transducer scans linearly across the anatomical structures. 


\subsection{Ultrasound Image Modality/Modes}

Ultrasound imaging is utilized in a variety of applications such diagnosing hip-joints or kidney stones, but Silver [21] mentions that fundamental principles remain the same, such as the propagation of sound waves into an anatomical structure and the recording of reflected echoes by the transducers. Currently, there are two different popular image modalities for clinical applications, which will be discussed in detail below.

\subsubsection{A-mode}

Silver [21] explains that A-mode (or amplitude mode) consists of a single transducer that scans through an anatomical structure. As an echo bounces back to the transducer, the envelope is detected and subsequently displayed in a function of depth, as shown in Figure 2-5.

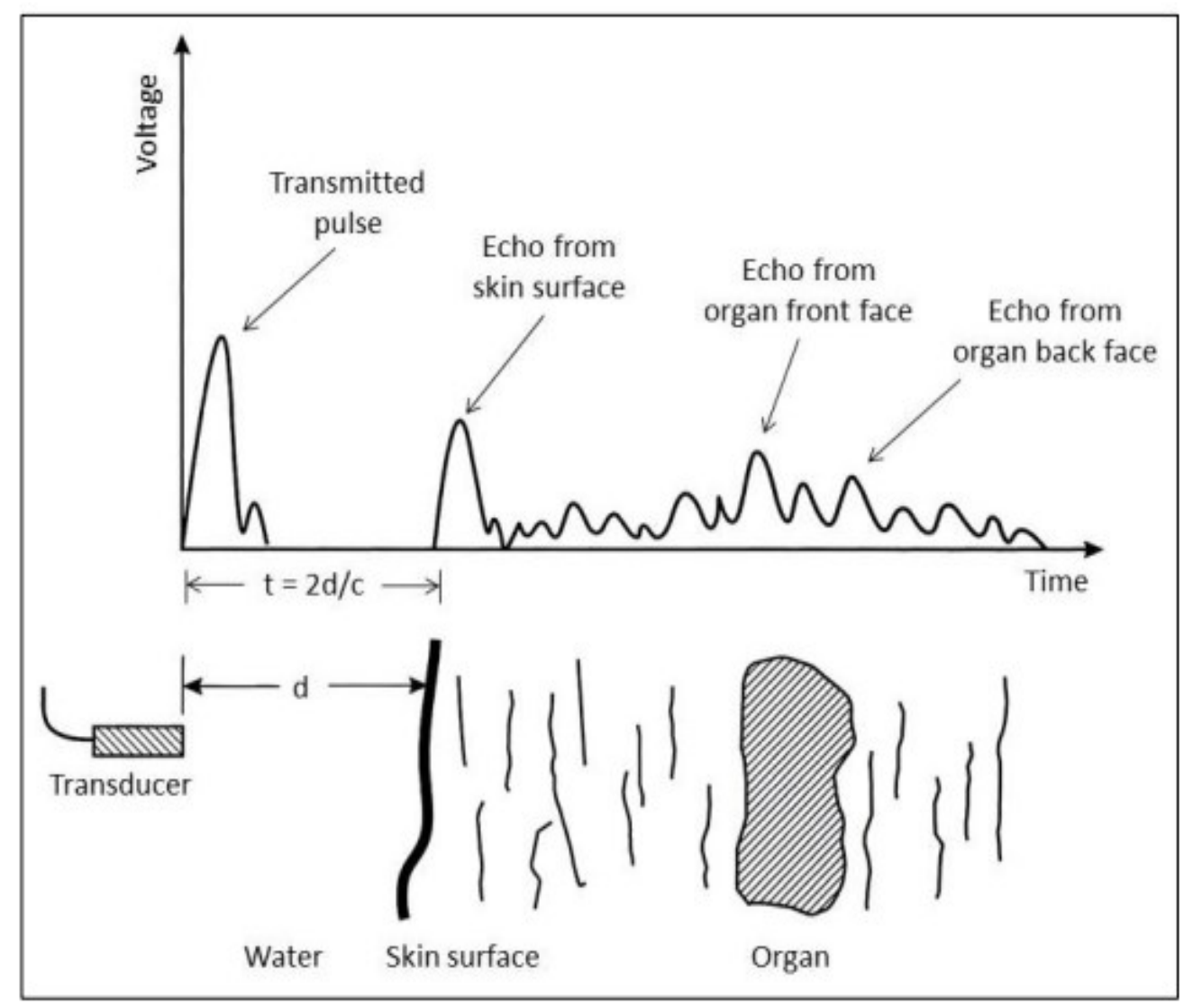

Figure 2-5: Detection of the envelope in A-mode ultrasound image from [29] 


\subsubsection{B-Mode}

Prince et al. [29] mention that the B-mode imaging or motion modality provides a 2D cross-sectional image of the internal tissues in the ROI. Data is collected by either moving the transducer in the elevation direction against the skin surface to acquire Amode scanlines as illustrated in Figure 2-1, or by moving a probe containing several transducers in an array to collect all scanlines in A-mode. After obtaining the A-mode signals, they are further modulated for brightness with echo intensities. In this thesis, Bmode is applied for elevation distance estimations.

\subsection{Usage in Ultrasound Musculoskeletal Imaging}

In myology from Rana et al. and Leroy et al. [30], [31], the ultrasound imaging system is used to examine muscular architecture in 3D to facilitate tendon transfer surgery. In nephrology from Elwagdy et al. [32], ultrasound imaging system is used to detect kidney stones. In obstetrics from Carneiro et al. and Dyson et al. [33], [34] ultrasound imaging system is used to detect developmental abnormalities fetuses.

According to Karadayi et al. [9], it is beneficial for medical professionals to have high resolution and real-time ultrasound images to obtain accurate diagnoses. A highfrequency transducer produces higher resolution images, but Fontaine et al. [35] explain that lower frequency linear transducers are preferred for scanning large muscular areas due to its ability to penetrate deeper into the tissue and provide the wider field of view than that with the higher frequency.

To better represent the anatomical structure in tissues or organs, it is advantageous to examine both the longitudinal and traversal planes, as Fontaine et al. [35] explain, the 3D ultrasound system provides a three-dimensional interpretation of the size of musculoskeletal lesions and injuries. The 3D ultrasound system offers real-time analyses, high image resolutions and greater speeds with lower costs than any other image modality in the ROI.

According to Fenster et al. [36], The 3D ultrasound system allows the machine to interpret Sonographers' data instead of relying on professionals' past experiences of prior diagnoses due to interpreting the 3D aromatically structure mentally. Consequently, the 
3D ultrasound system reduces type I errors (a failure to detect something) and type II errors (false positives) in the diagnostic results.

\subsection{Limitations of the 2D Ultrasound System}

Bahramabadi and Fenster et al. [27], [36], [37] describe two main limitations of the conventional 2D ultrasound system: Firstly, varying degrees of sonographers' experience can lead to inconsistent and subjective results, including Type I and II errors, which can occur when the sonographers are sweeping the probe back and forth due to formation of the $3 \mathrm{D}$ aromatically structure mentally. Secondly, reproducing an image with the exact same location and orientation as the previous sweep is close to impossible, given the structures of the skeletal muscle and soft tissues. Therefore the conventional 2D ultrasound is not suitable for the following up studies and patient monitoring. Challenges such as these have led towards a greater interest in developing the $3 \mathrm{D}$ ultrasound imaging system.

\subsection{Ultrasound Speckle Statistic}

The speckle pattern is considered as a type of noise as it only exists in ultrasound B-mode images. From Bahramabadi [27] techniques to disregard the effect of the speckle patterns are referred to as "speckle reduction," or "de-speckling."

However, speckles are considered in this thesis to be non-random noise, as speckle patterning remains the same when an object has been repeatedly scanned with the same transducer and configuration. This means the speckle signals are reproducible and nonrandom. In support, Nock et al. [38] describe the information which speckle signals carry as possessing the physical characteristics of both medium and transducer such as the dynamic range and noise.

As mentioned in Section 2.2.2, the speckle is dependent on the arrangement of the scattering. Ultrasound echoes scatter in various directions if the size of the particle is smaller than the wavelength of ultrasound. In addition, each tissue type has a particular speckle pattern associated with it due to the different arrangement of scatters. The speckle patterns have in fact been used widely for analyzing the tissue characteristics, and Thijssen et al. [39] utilize them to analyze the lesion or damage from the injury on the tissue. 


\subsubsection{Mathematical Model of Speckles}

An objective of this thesis is to determine the displacement between two image planes based on the change of the backscattered signal at two different positions. Afsham et al. [40], [41] describe how reflected signals obtained from transducers have information from both the medium and the ultrasound beam.

According to Afsham et al. [40], [41], one characteristic of the ultrasound beam is that it remains constant during use. Hence, a change in the medium from the change in speckle interprets the image plane motion for estimating the elevation distance.

Multiple reflections occur when the reflected signal from the specific scatter collides with other scatters on the way back to the transducer in the ultrasound system. Bahramabadi [27], assumes that multiple reflections occur but the amplitudes of the reflected signals are small enough to be ignored. Insana et al., $\mathrm{Ng}$ et al. and Jensen et al. [42]-[44] describe this assumption as the weak scattering condition, whereby the ultrasound imaging can be modeled as a first-order linear system. As shown in Equation 2.5 , the wave is propagated in the axial $(\mathrm{y})$ direction in the image plane coordinate $(\mathrm{P})$ within the linear probe in Figure 3-3.

$$
Q(\mathrm{x}, \mathrm{y}, \mathrm{z})=(h * \varsigma)(x, y, z)
$$

In Equation 2.5, $\mathrm{h}$ depicts the Point Spread Function (PSF) of transducer and $\varsigma$ represents the scatter function of the medium. $\mathrm{Ng}$ et al. and Jensen et al. [43], [44] describe the value of $h$ and point spread functions as dependent on the specification of the transducer. In this case, $\mathrm{h}$ is assumed to be the pulse. It can be a sinusoidal wave with zero means modulated by a Gaussian envelope. A resolution cell is also known as the envelope of traducer's PSF. The scatter function represented by $\varsigma$ is the sum of all scatters represented by $\mathrm{N}_{\mathrm{s}}$. It is listed as Equation 2.6.

$$
\varsigma(x, y, z)=\sum_{i=0}^{N x} e_{i D i r a c}\left(x-x^{(i)}, y-y^{(i)}, z-z^{(i)}\right)
$$

The Dirac means the Dirac impulse response function in Equation 2.6. The strength or echogenicity is represented by $e_{i}, x^{(i)}, y^{(i)}$ and $z^{(i)}$.The location of scattering $i$ is represented by $x, y$ and $z$. The density of scatters at the given resolution cell with respect to the incident wave within the transducers is represented by first order statistic of Q. If there is 
a uniform distribution in the space, the strength and echogenicity are independent in location. Goodman [45] described that the first order statistic $\mathrm{Q}$ has a zero mean.

If it becomes denser by having more scatters within the resolution cell, there is a larger Q with zero mean in the Gaussian distribution according to the central limit theorem. According to Goodman [45], this context is known as Rayleigh scattering or statistics. The speckle pattern derived from this condition is known as Fully Developed Speckle (FDS).

Wagner et al. [46] mentions that the second order statistic represents the relationship as ultrasound signal samples with a function of their respective locations. Prager et al. [47] defined that the first order may not be able to handle the complicated signal samples since they are statistically independent. If the current resolution cell with a finite size is located in a way that its center is close to the next resolution cell, they overlap in space. Hence, the image has the similar scatters. It leads to signal samples between these two images that are mutually correlated. Consequently, it concludes that the correlation decreases when there is more overlap between resolution cells' width in a function of distance. This concept is also known as speckle decorrelation.

The second order statistics of the ultrasound speckles are defined as the autocorrelation function and applied in ultrasound speckle tracking. It is dependent on the transducer's characteristics only under the Rayleigh scattering conditions or fully developed speckle (FDS). Under this circumstance of Rayleigh scattering as described by Wagner [46], the speckle cell size is proportional to the resolution cell size.

The speckle cell size in the axial direction is an inverse function of the pulse bandwidth. The speckle cell size in the lateral direction is proportional to the size of the transducer beam width.

As long as the resolution cell has a similar shape to the Gaussian distribution according to the explanations from Prager et al. and Ng et al. [44], [47], the normalized autocorrelation function or decorrelation with the echo intensity signal can be represented in the function of the elevation distance. Chen et al. [48] mention that the Gaussian function provides a great approximation to the point spread function for the properties of transducers.

\subsubsection{Correlation Formula}


From Wagner [46], the resolution cell as a series of the ultrasound pulses transmitted and received by the probe at a point of time to reproduce the image intensity. In practice, it can be difficult to reliably observe if the scatters are occupying the same resolution cell or not, but observing overlap of speckles in the resolution cell on the image is in a different scenario. Prager et al. [47] reason that the distance between two overlapping resolution cells depends on the amount of correlations between two received signals. In this case, the amount of correlations is also associated with the amount of overlap between the resolution cells, and the autocorrelation function or decorrelation curve can be found for B-mode ultrasound images.

Gee et al. [16] describe how this is done by utilizing a Pearson correlation coefficient for Rayleigh scattering or the fully developed speckle (FDS). As listed in Equation 2.7, $\mathrm{I}_{1}$ and $\mathrm{I}_{2}$ represent the backscattered intensity signals for patch one and patch two respectively. The associated backscattered amplitudes are listed as A1 and A2 in Equation 2.8.

$$
\begin{gathered}
\rho\left(I_{1}, I_{2}\right)=\lambda^{2} \\
\mathrm{P}(A 1, A 2)=\frac{4 E\left(\lambda^{2}\right)-2\left(1-\lambda^{2}\right) K\left(\lambda^{2}\right)-\pi}{4-\pi} \\
\lambda^{2}=\exp \left(\frac{-\delta^{2}}{2 \sigma^{2}}\right)
\end{gathered}
$$

Gee et al. [16] indicate that $\mathrm{K}$ and $\mathrm{E}$ represent the complete elliptic integrals of the first and second kind respectively. They are based on an assumption of the resolution cells in a Gaussian shape and decorrelation curve in a Gaussian function. Thus, the parameter $\lambda$ is based on the standard deviation of the elevation cell width as listed in Equation 2.9. The elevation distance separation of patches is represented by $\delta$. There is a relationship between the elevation distance $\delta$ and correlation $\rho$. The resolution cell width $\sigma$ is not the same across the scan planes. The resolution cell width varies in the B-mode images while scanning in the elevation direction. It is more obvious to realize the change in the axial direction for the resolution width since it varies more with respect to the depth. It is thus necessary to obtain the different correlation curve at a given position of the scan plane. 
Gee et al. [16] mention two alternative methods for obtaining the correlation curve. First, the elevation separation distance is obtained by applying the theory of acoustic physics for different positions of scan planes according to the specifications from manufacturers of transducers. Second, the calibration is done by the decorrelation curves for a specific ultrasound probe through scanning a speckle phantom. The Pearson correlation coefficient $\rho$ is then computed directly from the ultrasound B-scan image for the various elevation offsets $\delta$.

\subsubsection{Speckle for Out-of-Plane Motion Estimation}

As mentioned before, the decorrelation curve is independent from the medium according to the Rayleigh scattering condition. The elevation distance between two different ultrasound images obtained next to each other by the same transducer is estimated with second order statistics from FDS patterns. In practice, the speckled phantoms are created by containing multiple random ultrasound scatters.

Gee et al. [16] mention that the Cambridge heuristic method can be used if both coherent and Rayleigh scattering conditions are held. By scanning with known elevation intervals, a transducer specific decorrelation curve can be obtained. The elevation separation distances can also be estimated from a known decorrelation curve or viceversa. In this thesis, the speckle phantom is scanned to obtain the decorrelation curves for the elevation distance estimation from a set of images. 


\section{Chapter 3: State of Art}

\subsection{Acquisition Techniques for 3D Ultrasound System}

Fenster et al. and Prager et al. [37], [49] describe various methods to acquire the 3D ultrasound images, including 1D and 2D transducers. The similarities lie with the utilization of the probes that sweep across the anatomical structures while simultaneously collecting the $2 \mathrm{D}$ images. The challenge is now to figure out the orientations, positions and intensities of the 2D images within the 3D image coordinate system.

\subsubsection{Acquisition Technique from Mechanical Sweep Probe}

The mechanical probe consists of a motor and a 1D array transducer which is displayed in Figure 3-1a. Karadayi et al. [9] describe how the data is collected then rendered into 3D images during the acquisition process when the motor rotates, tilts, or translates.

Rohling et al. [50] mention that the mechanical probe produces the various volumes based on the angles in accordance with constraints imposed by the 1D transducer. Prager et al. [49] explain that the mechanical probe is advantageous in that it provides a greater resolution to the ROI during the data acquisition process from the various ultrasound acoustic waves found in the 1D transducers. The disadvantage of the mechanical probe is the limited viewing angles of the $1 \mathrm{D}$ transducer. 


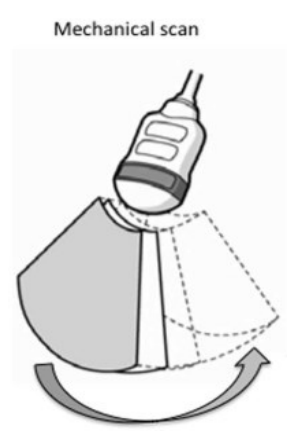

$2 \mathrm{D}$ array scan

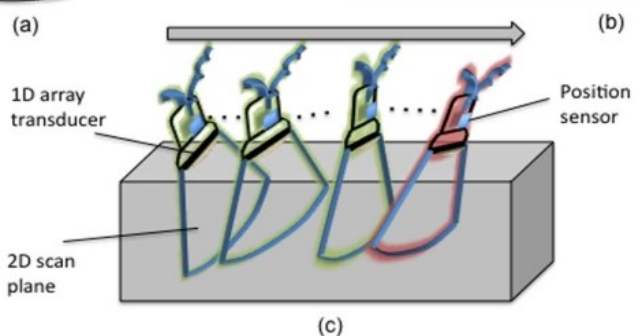

Figure 3-1: Various acquisition techniques for 3D US image system(a) Mechanical scan with 1D array transducer(b) 2D array scan(c) Freehand scan from [9]

\subsubsection{D Array Transducer's Acquisition Technique}

The 2D array transducer operates in synchrony with the ultrasound probe's sweeping motion. Karadayi et al. [9] mention that the ultrasound beams from the probe have a pyramidal shape as shown in Figure 3-1b. An advantage to having the $2 \mathrm{D}$ array transducer is its simultaneous data acquisition and processing resulting in greater time efficiency for computing the $3 \mathrm{D}$ volume. The disadvantage with the $2 \mathrm{D}$ array transducer is the higher cost when compared to the mechanical probes.

\subsubsection{Freehand Scanning's Acquisition Technique}

In the freehand scanning acquisition technique, probes are swept across the area of interest as illustrated in Figure 3-1c. Karadayi et al. [9] detail that the 2D B-mode images are sequentially constructed with positions and orientations that are recorded simultaneously. The coordinate system including image planes $(\mathrm{P})$, reconstruction volume $(\mathrm{C})$, transmitter $(\mathrm{T})$ and receiver $(\mathrm{R})$ for freehand scanning technique is shown in Figure 3-2.

The freehand scanning acquisition technique can involve ultrasound probe with or without a tracking device. There are two advantages and one disadvantage with the 
freehand scanning technique when comparing its acquisition techniques to the mechanical probe scanning technique and the $2 \mathrm{D}$ array transducers technique.

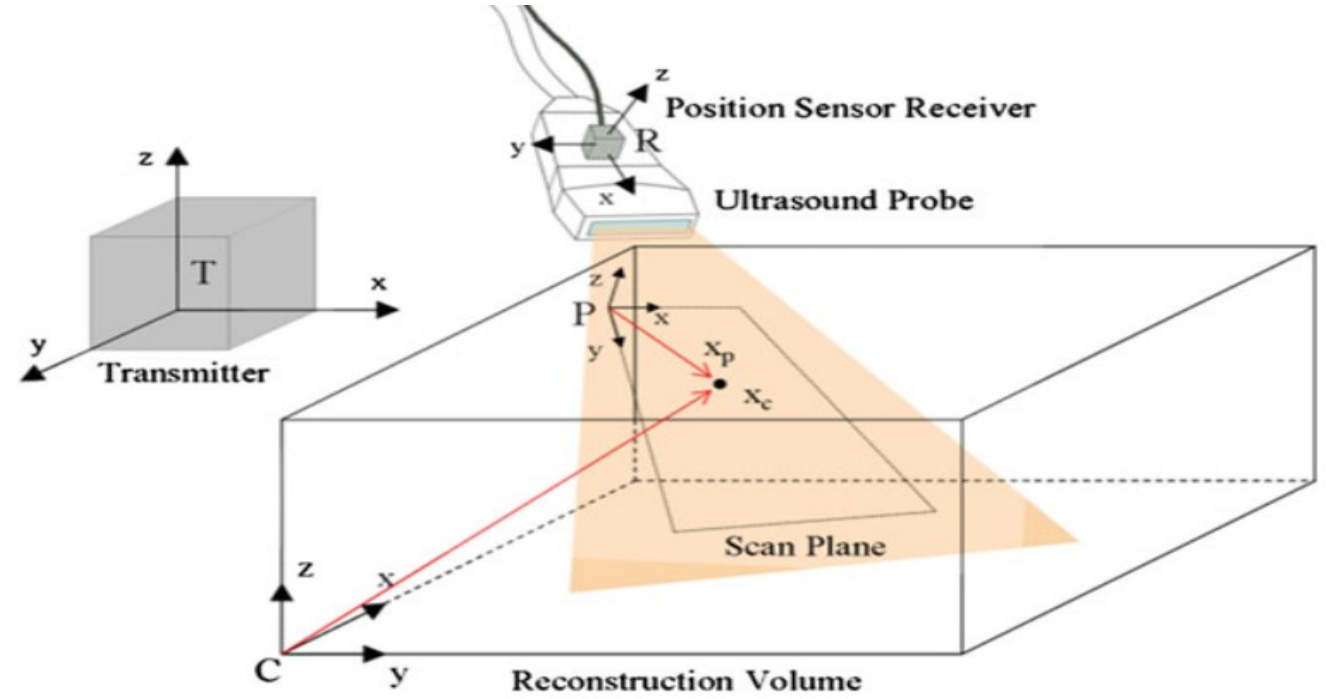

Figure 3-2: Coordinates for the EM trackers from [51]

Karadayi et al. [9] note that the freehand scanning technique is both cheaper, and more flexible in rendering any arbitrary volume. However, the technique makes it difficult to remain stationary at one ROI within the anatomical structure so it is unsuitable for patient monitoring.

\subsubsection{Freehand Scanning's Acquisition Technique with Tracking Device}

In the freehand scanning technique with the tracking device, Karadayi et al. [9] explain that a six-degrees-of-freedom (DOF) position sensor or tracker is attached to the probe which contains a 1D transducer array. Gee et al. [16] reflect that a disadvantage of the freehand ultrasound scan with the tracking device is its time inefficiency for the calibrations and complicated setup procedures when compared to performing the same scan without tracking device. However, scanning with a tracking device permits a greater accuracy due to the position sensors that provide real-time status updates via temporal and spatial calibrations. 


\subsubsection{Freehand Scanning's Acquisition Technique without Tracking Device}

In the freehand ultrasound scanning technique without the tracking device, Prager et al. and Karadayi et al. [9], [49] indicate that there are no position sensors attached to the probe containing a 1D array transducer. The data is collected in 1D then reconstructed into 2D B-mode images while the probe is sweeping. To estimate the elevation distance of the B-mode ultrasound images, the Fully Developed Speckle (FDS) in the current frame of the image is compared with that of the next frame by computing its correlation coefficient. However, Gee et al. and Karadayi et al. [9], [10] are quick to note that the accuracy in FDS still requires improvement.

The advantage of the freehand ultrasound scan without the tracking device is its timesaving operation and easier setup procedures compared to the freehand scanning with the tracking device. The freehand ultrasound scan without the tracking device does not require position sensor to be calibrated with the ultrasound machine thus it becomes an attractive option for clinicians and researchers.

\subsection{Components of the 3D Freehand Ultrasound System}

As explained previously, Dewi et al. and Karadayi et al. [9], [51] describe how the series of B-mode ultrasound images are recorded with the presence of position sensors in the 3D freehand ultrasound system. Prager et al. and Dewi et al. [49], [51] explain that the positions and orientations of sensors with respect to each frame are labeled to facilitate the subsequent calibration process. The $3 \mathrm{D}$ freehand ultrasound system comprises a computer system, an ultrasound machine, and a tracking system: The purpose of the computer system is to record the data from the tracking device; the function of the ultrasound system is to collect the 1D data and integrate it into 2D B-scan images which are known as the construction process; and the tracking system consists of a mechanical arm and tracking devices.

From Bahramabadi [27] the ultrasound probe is attached to the mechanical arm with six DOF. The precise position can be calculated by knowing its geometric property so higher accuracy can be achieved. However, there are three disadvantages of the mechanical arm: it tracks only one anatomical structure at a time, its price, and the complexity of its operations. 
Prager et al. and Dewi et al. [49], [51] list two types of tracking devices: electromagnetic trackers, and optical sensors. From Raab et al. [52], electromagnetic tracking devices consist of transmitters which emit a magnetic field, and sensors which measure the strength of the magnetic field emitted by the transmitter. The changes in electromagnetic measurements detected by each sensor provide an estimate of its position and orientation with respect to the transmitter.

An advantage of the electromagnetic sensor when compared to optical technologies, lies with the fact that it does not require a clear sight line between sensors and the transmitter. However, a disadvantage is that metallic objects in the surrounding area during operation may affect results.

With regards to the optical sensor, Prager et al. and Dewi et al. [49], [51] described at least two digital cameras are needed for calculating the positions in $3 \mathrm{D}$ since depth information is provided from the second camera. Each camera only captures the 2D positions. An advantage of the optical sensor is its ability to detect the absolute position of the target with respect to each camera's coordinate system.

A disadvantage of the optical sensor found in the tracking system is its requirement to have a clear sight line between the camera(s) and the probes. This deficiency can be mitigated by installing more cameras around the target in the ROI in order to increase the clarity of the sight line. However, an additional disadvantage involves the logistics of having to install more camera(s) with additional expense and loss of portability.

\subsection{Calibration Process}

After sequentially collecting the 2D-ultrasound images in the image plane $(\mathrm{P})$ coordinate system as illustrated in Figure 3-2, the calibration of the collected data from the ultrasound system follows. From Nelson and Pretorius [50] the next step is the reconstruction of the $3 \mathrm{D}$ volume of the anatomical structure.

There are two requirements of the calibration process. First, the position of each pixel of an image must be correctly placed into the overall 3D Cartesian coordinate system containing X,Y and Z-axes. Second, probes with the tracking device synchronize both the position and image data by labeling the beginning and ending of the frame on all components. Gibbon et al. [53] mention that calibration has a significant effect on both 
the quality and accuracy of the reconstruction algorithm which will later be detailed in Section 3.4 .

\subsubsection{Temporal Calibration}

From Bahramabadi [27], the temporal calibration is for matching the ultrasound images with the position reading sensors in time. It deals with the latency of both the ultrasound machine and position sensing device so that time offset between the start and end of the frame in each data set can be calculated. The offsets are assigned as time steps to the data input streams located in both the tracking devices and the image system. In the imaging system, the offsets are derived from the frame-grabbing hardware which issue time stamps automatically. The latency is calculated by obtaining the difference between the data in the position sensors and frame-grabbing hardware, and vice versa.

By providing the step input, Prager et al. [54] indicate that a rapid change occurs if the starting positions for both position sensors and image streams happen at the same time. Hsu and Mercier et al. [55], [56] mention that the step input is defined by holding the probe firmly and sweeping then jerking away from the skin surface suddenly. From this, the time difference between the positions of the tracker's reading streams and the image streams of the ultrasound machine results in the correct timing.

\subsubsection{Spatial Calibration}

Prager et al. [54] described that the spatial calibration matches the time stamps between the image and position data by determining the six DOF's rigid transformations found between the receiver $(\mathrm{R})$ and the image plane $(\mathrm{P})$ coordinate systems in Figure 3-3.

There are two different ways to perform spatial calibrations. Prager et al. [57] use two crossing wires as the center position of the volume reconstruction with 12 unknown values. The advantage of using two crossing wires than three crossing wires is the increase in accuracy with the lesser unknown values. The disadvantage is the increased implementation complexity due to challenges in determining the center crossing-point.

Prager et al. [57] mention that the second way is to use three orthogonal wires which represent the center position of the volume reconstruction with 14 unknown values. The advantage of using three crossing wires is in its simplicity when scanning the length of the wires. The disadvantage is in decreasing the implementation's accuracy due to more 
unknowns. Overall, Pagoulatos et al. [58] claim that the disadvantage for the spatial calibration is the collaboration time, as the entire process lasts approximately two minutes.

As shown in Figure 3-3, there are four coordinate systems in the freehand 3D ultrasound system. Prager et al. [57] mention that the first coordinate system is the volume reconstruction (or global coordinate system) and is denoted by the letter $\mathrm{C}$. The second coordinate system is the image plane and is denoted by the letter P. The third coordinate system is the transmitter (or source) and is labeled with the letter T. The fourth coordinate system is the receiver (or sensor) which is attached to the probe and denoted with the letter R.

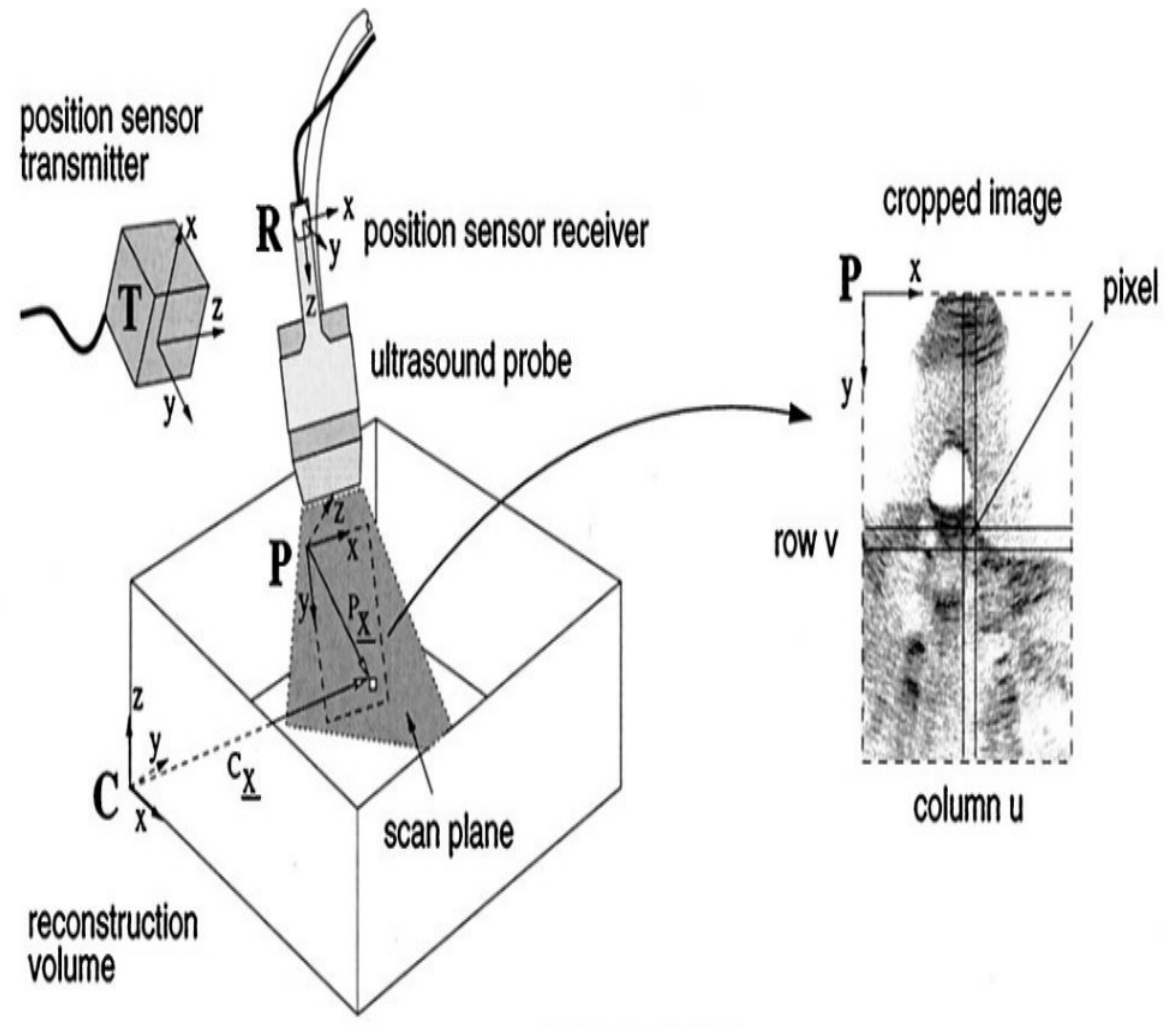

Figure 3-3: Various coordinate systems including image plane (P), Receiver (R), Transmitter (T) and Volume Reconstruction (C) from [57]

There are many transformations used to obtain the final image pixel to the global coordinate system in 3D. Such transformations are in sequence starting from the $\mathrm{P}$ coordinates to the $\mathrm{R}$ coordinates to the $\mathrm{C}$ coordinates. 


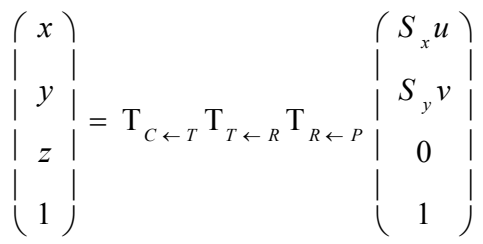

Prager et al. [57] explain that the symbol of $\mathrm{T}_{\mathrm{j} \leftarrow \mathrm{i}}$ in Equation 3.1 signifies a transformation from the $\mathrm{i}$ coordinate system to the $\mathrm{j}$ coordinate system. Both the symbols $\mathrm{Sx}$ and Sy represent scale factors with the units in $\mathrm{mm} /$ pixels. In Equation 3.1, the transformation matrix represents the stepwise process for obtaining the coordinate system of the volume reconstruction. The data collected in the experiment from the position sensor is utilized in transformation matrix of $\mathrm{T}_{\mathrm{T} \leftarrow \mathrm{R}}$. The next step is to reconstruct the $3 \mathrm{D}$ images from the locations of the $2 \mathrm{D}$ images.

\subsection{D Reconstruction Algorithm}

After both the temporal and spatial calibrations are completed, Hsu et al. [11] mention that it is time to reconstruct the volume for mapping the positions, orientation and intensity of an image pixel in the 2D spatial domain to the voxel in the 3D spatial domain. From Solberg et al. [18], there are different methods for assigning the intensity value of voxels in the $3 \mathrm{D}$ coordinate from the intensity value of the pixels in the $2 \mathrm{D}$ coordinate for the freehand 3D ultrasound system. They are the voxel-based method (VBM), pixel-based method (PBM), and Functional Base Method (FBM).

\subsubsection{Voxel-Based Method (VBM)}

From the data acquisition process, VBM reconstructs the voxel grid from 2D images, which include the voxel nearest neighbor (VNN). Rohling et al., Prager et al. and Solberg et al. [18], [54], [59] indicate that the voxel value is assigned to the nearest neighbor of surrounding pixels according to the shortest distances between each voxel and its neighbors.

Coupé et al. [60] explain that a disadvantage of the VNN is its inability to reduce the speckle noise emanating from the transducers due to the direct assignment of the voxel 
value from its neighbors. However, an advantage of VNN is the simplicity of its implementation.

Prager et al. [61] mention how the Stradx system developed by Cambridge University is an example of VNN interpolation implementation. The Stradx system arbitrarily reconstructs the oriented $2 \mathrm{D}$ slices through a set of $2 \mathrm{D}$ images rather than creating a $3 \mathrm{D}$ volume. It goes through each pixel within the targeted 2D slice and assigns the voxel value. When compared to VNN, the Stradx system provides both the real-time acquisition and visualization capabilities. Alternatively, there are different approaches in the voxelbased algorithm. Coupé et al. [60] explains that they are the voxel-based method with interpolation (VBMI) and the probe trajectory (PT).

\subsubsection{Voxel-Based Method with Interpolation (VBMI)}

Entrekin et al., Trobaugh et al., Thune et al. and Berg et al. [62]-[65] performed VBMI by going through input pixels within a volume and finding the two nearest $2 \mathrm{D}$ slices to determine the final voxel value based on its 1D interpolation. Coupé et al. [60] list two interpolation methods which are dependent on the maximum angle between two enclosed scan planes which are both perpendicular and parallel in the probe. If the maximum angle is less than twenty degrees, the orthogonal linear interpolation in the nearby scan planes is applied. Voxel values are determined from the closest planes.

Solberg et al. [18] mention that if the maximum angle is greater than twenty degrees, a virtually middle plane is created through the center of the voxels. In addition, Coupé et al. [60] determined the voxel value with the calculation of the orthogonal linear interpolation in pixels nearest to planes that are both perpendicular and parallel. If there is more than one closest plane, the average between the two planes is applied. The closest distance is calculated through inverse distance weight.

Trobaugh et al. [63] introduce a way to find the two nearest neighbors surrounding pixels by scanning through a voxel and determining the two nearest $2 \mathrm{D}$ slices on each side of the voxel. First, the plane to the normal is calculated for each slice then the value is interpolated from the four surrounding pixels. The final voxel is then obtained through the weighting sum calculated from the distance to the nearest pixel. 
Trobaugh et al. and Coupé et al. [60], [63] instead employ the probe trajectory (PT) method by calculating the virtual plane determining the surrounding pixels from the shortest distance between the 2D image planes. However, a limitation of PT is the requirement to have a constant speed between virtual planes and the $2 \mathrm{D}$ planes. Thune et al. [64] posit a voxel-based tri-linear interpolation based on obtaining the raw data from the US system to enable the $3 \mathrm{D}$ reconstruction to take place from interpolating eight points in a scan line of the probe.

Rowling et al. [59] developed the distance weight algorithm (DW) to properly handle speckle noise from the transducer in both PT and VBMI. Here, a voxel is assigned by a set of surrounding pixels within a predefined range of the center voxel. In addition, Barry et al. and Huang et al. [66]-[68] mention that all pixels within the range are weighted

with the inverse of its distance to the voxel center. Hence, the DW works well in reducing the speckle noise but causes image blurring.

\subsubsection{Pixel Based Method (PBM)}

For PBM, Solberg et al. and Chen et al. [18], [69] explain that a voxel value is assigned by traversing each pixel in the 2D ultrasound image in two steps: distributed step (DS) or bin-filling, and the hole filling step (HFS). At the stage of DS, input pixels are traversed within an entire 2D image so each voxel value is assigned to multiple values according to pixel's weight. Then, in HFS, the empty voxel is filled while traversing all voxels.

\subsubsection{Distributed or Bin-filling Step}

Solberg et al. [18] note that the Pixel nearest neighbor (PNN) is proven to be the most popular method within the DS step. Here, each pixel in the 2D US image is entirely scanned then filled into the nearest voxel and the voxel value is assigned from averaging its neighbor pixels.

\subsubsection{Hole-filling Step}

After DS is completed, there are empty voxels inside the voxel array, and HFS becomes useful. At HFS, the volume of voxels is scanned through to identify the empty voxel, which can then be filled with a variety of methods. McCann et al., Hottier et al and 
Solberg et al. [18], [70], [71] describe how the value of a voxel is assigned according to the average, median, maximum or median of a non-zero voxel in a 2D plane.

Estépar et al. [72] developed PNN with a 3D kernel around filled voxels during the HFS within PBM. Solberg et al. [18] explain that the voxel value is evaluated by applying a weighting of the nearby neighborhood or kernel instead of filling the hole directly. One of the examples applied is the time-consuming Gaussian kernel which fills the voxel across its neighborhood, but depends on the value of the intensity of pixels near its neighbors. The size of the kernel is associated with numbers of intensity at a given dimension.

As a result, San José-Estépar et al. [73] introduced an adaptive spherical Gaussian kernel for the purpose of faster interpolation under PBM. An individual filled voxel is first applied to the neighboring voxel by utilizing the spherical Gaussian kernel. The size of the kernel in the PNN bin filling step determines the density of intensity, but ensuing the implementation complexity is a disadvantage. Barry et al. [66] developed the distance weighting function with the spherical kernel whereby input pixels are traversed and then assigned pixel values to the local neighborhood described as the kernel. Besides the pixel value, weight values are also available and are used to calculate the resulted voxel value by measuring the distance between all the neighboring pixels. Hence, the pixel and voxel values are not equivalent since the position of the pixel is used in the process. An advantage of distance weight is its faster computation time.

\subsubsection{Function Based Method}

In general, a polynomial function is selected in FBM for traversing through the input pixels. The coefficients of the polynomials determine the number of functions passing through pixels. Consequently, a voxel array can be created from functions at regular intervals. Rohling et al. [59] use the spline method to approximate data for volume reconstruction. The smooth function is used as an interpolation function to evaluate the volume by dividing the data into various segments, which reduces computation time. Sanches and Marques et al. [19] mention the low resolution of voxel volume at the first iteration by applying eight nodes of its neighbors to calculate the final voxel value. Sanches 
et al. [74] also describe the statistical reconstruction and interpolation methods as Rayleigh distribution.

Solberg et al. [18] note that the limitation for the VBM is its real-time construction since it cannot reconstruct until all the data has been collected. The advantage of the PBM is with its real-time data acquisition and visualization when the volume reconstruction and data collection takes place simultaneously. Issues arise when filling the empty holes during the HFS. However, computation time can be used to estimate the algorithm's performance.

VNN permits the fastest reconstruction among all the other methods by assigning voxel intensities from its pixel neighbors, though it sacrifices accuracy. However, the size or dimension of the kernel can reduced to decrease VNN computation time. To do so, Barry et al. [66] employed the 1D kernel to reduce computation time, which resulted in a lower accuracy. Solberg et al. [18] explain that the advantage of the FBM is in creating the smoothing volume based upon the voxel intensity through its large area of pixels, which improves visual appearance. Reconstruction quality ultimately depends on position sensor accuracy, probe calibration, and tissue movement.

\subsubsection{Position Sensor Accuracy in 3D Reconstruction}

Solberg et al. [18] warn that a blurred volume can occur due to inaccuracies in the position tracking operation or tissue movement even when the compound volume appears accurate. In this case, it is preferred to use a simple algorithm rather than a complicated one for increased efficiency. To obtain the maximum quality, the algorithm selected must be dependent upon both the position accuracy and data collection.

\subsubsection{Probe Calibration in 3D Reconstruction}

As mentioned previously in Section 3.3, Charbonnier et al. [75] detail that probe calibration is dependent upon matching the coordinate system between tracking devices and the ultrasound machine system. Lindseth [76] caution that if there are inaccuracies during the probe calibration process, reconstruction accuracy will decrease. 


\subsubsection{Tissue Movement in 3D Reconstruction}

Sweeping the probe against the skin generates pressure and moves tissue vertically and horizontally. As a result, Treece et al. [77] introduced the pressure compensation algorithm to increase the accuracy during the estimation of the elevation distance in the 3D freehand ultrasound system.

Another factor relating to the image quality and computation time must be considered: Due to the hardware restriction, Solberg et al. [18] note a longer computation time between real-time and high-quality images with any complicated algorithm.

Gobbi et al. [78], [79] implemented a multi-process and multi-thread algorithm to display anatomical structures in 3D and improve calculations of the positions and orientations of the 3D anatomical structure. However, Solberg et al. and Gronningsaeter et al. [18], [80] observe that a primary constraint for the US in 3D reconstruction is its speed and quality. The commercial product made by MISON can reconstruct the 3D volume in 30 seconds with acceptable quality even though its method is not published.

\subsection{Visualization}

Birkeland et al. [81] list four main pipelines for displaying ultrasound images as depicted in Figure 3-4.

\subsubsection{Preprocessing}

Pre-processing steps consist of data acquisition, calibration, and volume reconstruction, as introduced in Section 3.1, Section 3.3 and Section 3.4.

\subsubsection{Segmentation}

In segmentation, Birkeland et al. [81] state how the inset image inside the ROI is selected but the insets outside the ROI are ignored.

Carneiro et al. [33] introduce a technique for automating the segmentation of the brain, whereby the automated algorithm can locate the cerebellum so the input data required to search is narrowed down.

\subsubsection{Rendering}

The goal of rendering is to depict the voxel value on the monitor. To do this, Nelson et al. [5], [82] introduced surface volume rendering to display the ultrasound data in 3D. 


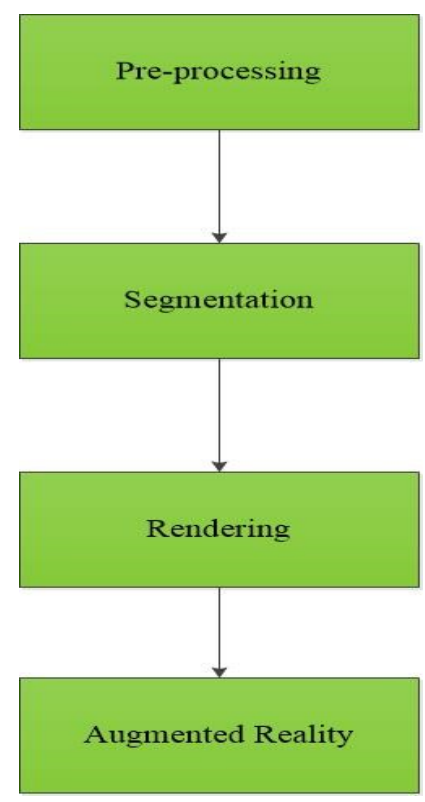

Figure 3-4: Visualization pipeline. Reconstructed from [81]

Nelson et al. and Ralston et al. [83], [84] explain that the volume rendering is used to map the voxels including their colors and positions into the 3D space in the display. Volume rendering does not require polygons or patches in advance. A limitation of the volume rendering is its requirement for the collection of all the image properties before the next rendering. During the process of rendering, each color from the image contributes to the final voxel color. Hence, the final color of the voxel is dependent on the color and opacity of the current pixel and its neighbors.

The most widely used rendering algorithm is based on ray-casting. In one study, Carneiro et al. and Gorkin [33], [85] performed ray-casting scans through all the image pixels to find its associated color and opacity. Weiskopf et al. describe four steps in volume ray-casting as illustrated in Figure 3-5 [86]. First, a ray is cast through a volume containing the voxels. Second, voxels along the firing ray are selected and interpolated in order for the selected voxels to align properly. Third, shading occurs where each voxel is assigned to a specific transparent value. Fourth, compositing happens when the final color value on the sight of the ray is determined from its pixel surrounding. 

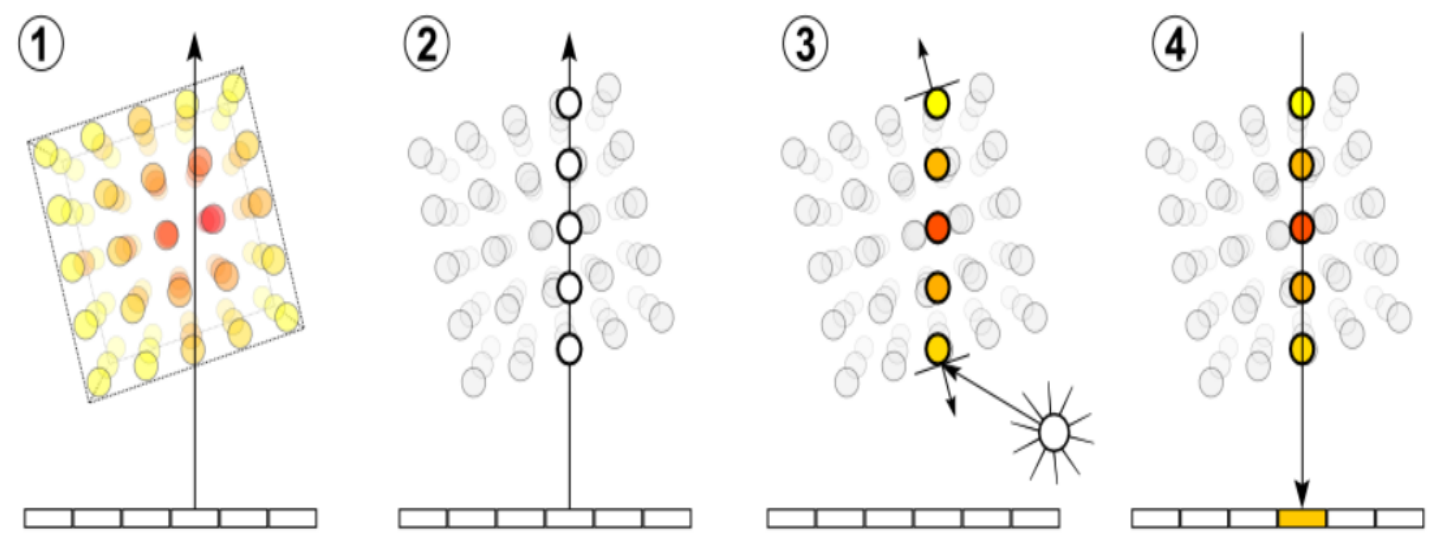

Figure 3-5: Procedures for the volume ray-casting from [85]

Glassner [87] mentions that ray casting can be limited as it deals with a single ray source and does not consider any effects from others. Therefore, a technique named raytracing is introduced for synthesizing the more complicated and realistic 3D images. For example, in Figure 3-6, there are two light sources: points A and B emitting rays. In ray La, it can be completely traced back without any interference. With ray Lb however, the light source has been blocked by sphere $\mathrm{S}$. Consequently, only ray La can reach point $\mathrm{P}$.

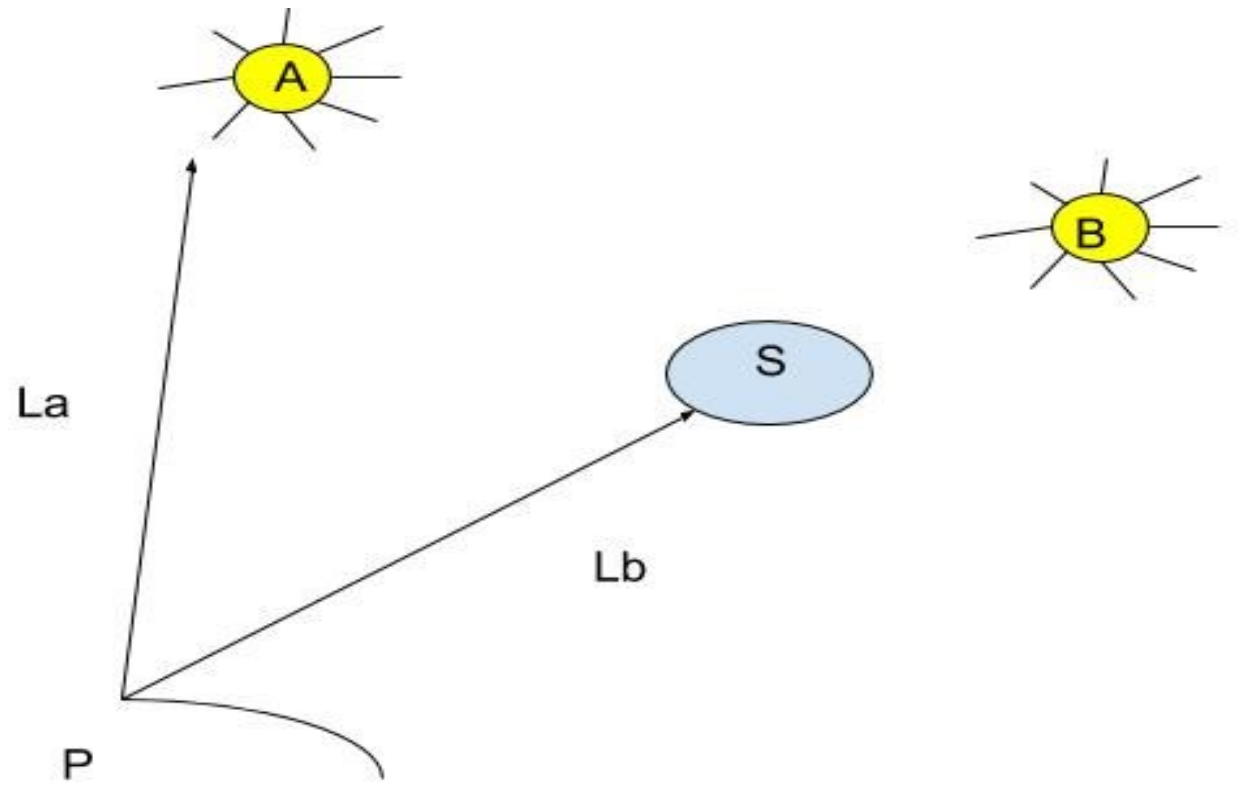

Figure 3-6: Raytracing with two light sources. Reconstructed from [87] 


\subsubsection{Augmented Reality}

With regards to Augmented Reality (AR), Birkeland et al. [81] describe how AR is required because of difficulties associated in observing how 3D ultrasound images relate to the spatial position of the human body after reconstructing the 3D ultrasound image. AR can superimpose various 3D models from $2 \mathrm{D}$ ultrasound images into recorded video clips. In support, Sato et al. and Belohlavek et al. [88], [89] note that it helps surgeons to see the location of the ultrasound images inside the body, which reduces the risk of errors in surgery.

\subsection{Distance Estimation for the Sensorless Out-of-plane Motion}

As mentioned in Section 3.1.3, one of the main concerns for the freehand 3D ultrasound system is the requirement of having tracking devices mounted on the ultrasound probe. Consequently, researchers have been investigating ways to remove tracking devices through speckle tracking. Recently, Afsham et al. [40], [41] applied speckles in the images to estimate elevation distances between frames in the 3D coordinate system.

Szabo [23] refers to the in-plane and out-of-plane motion distance estimations, which are two methods that utilize speckles for distance estimation.

\subsubsection{Speckle with In-plane Motion Distance Estimation}

Housden et al., Afsham et al. and Prager et al. [40], [41], [47], [90], [91] note that the speckle with in-plane motion estimation applies the image registration technique with either the block matching or radio frequency (RF) tracking algorithm. The block matching algorithm divides an image into various blocks, and the ensuing correlation coefficient is calculated between each block with the next moved block placed in the either the vertical or horizontal plane. A main disadvantage again lies with the complicated algorithm's lengthy computation time.

The RF tracking method calculates the correlation coefficients between the signals at various frames. In clinical settings, the RF's limitation is its non-availability. Accuracy can be improved by removing pressure artifacts as mentioned in Section 3.4.4.3.

\subsubsection{Speckle with Out-of-plane Motion Distance Estimation}

The speckle with the out-of-plane motion distance estimation is very challenging due to its diminished accuracy. Afsham et al. [40], [41] list two main methods for the out-of- 
plane distance estimation: the correlation-based tracking method, and the regression based method.

\subsubsection{Correlation Based Tracking}

Gee et al. [16] note how widely adopted the correlation-based tracking technique is due to the ease of its implementation. As illustrated in Figure 3-7, there are images A and B with frame $\mathrm{n}$ and $\mathrm{n}+1$ respectively. The in-plane motion includes the translation in both $\mathrm{x}$ and y directions and rolls along the normal of the image plane.

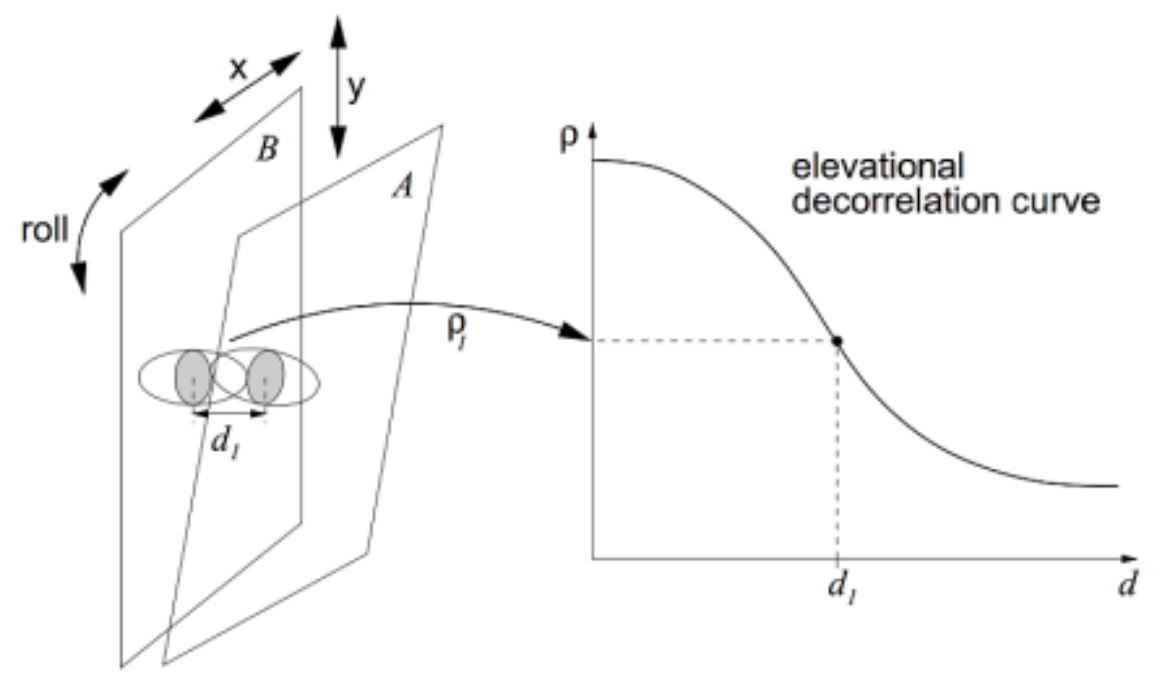

Figure 3-7: Concept of the elevation speckle decorrelation curve from [16]

Gee et al. [16] explain that the definition of the out-of-plane motion is found between images $\mathrm{A}$ and $\mathrm{B}$ and include the translation in the elevation direction, pitch around the lateral axis, and yaw around the axial axis. To estimate the distance in all three directions, it is necessary to estimate the correlation between various patches in both images A and $\mathrm{B}$ due to the imperfection of elevation focus in the ultrasound probe as illustrated in Figure 3-8. Therefore, there will be a distance between the images.

Gee et al. [16] mention that the patches containing the scatters overlap the region of the resolution cells which determine the correlation coefficients of the two images, from which in turn the elevation distance can be derived. If there are more crossing sections between the overlapping regions, the correlation coefficient is going to increase. If the separation distance in the elevation direction becomes wider, the correlation coefficient decreases. By taking this into account, the elevation distance can be found with the 
correlation coefficient between two patches with the decorrelation curve. The angles in tilt and yaw between images $\mathrm{A}$ and $\mathrm{B}$ can subsequently be found. One way to find the decorrelation curve for each probe in evaluating the resolution cell width is to obtain the detailed specification from the manufacturer. An additional option would be to scan the speckle phantom then measure the correlation coefficients from the ultrasound B-mode images with various elevation offsets. This second way is more popular and feasible for calibrating the decorrelation curve.

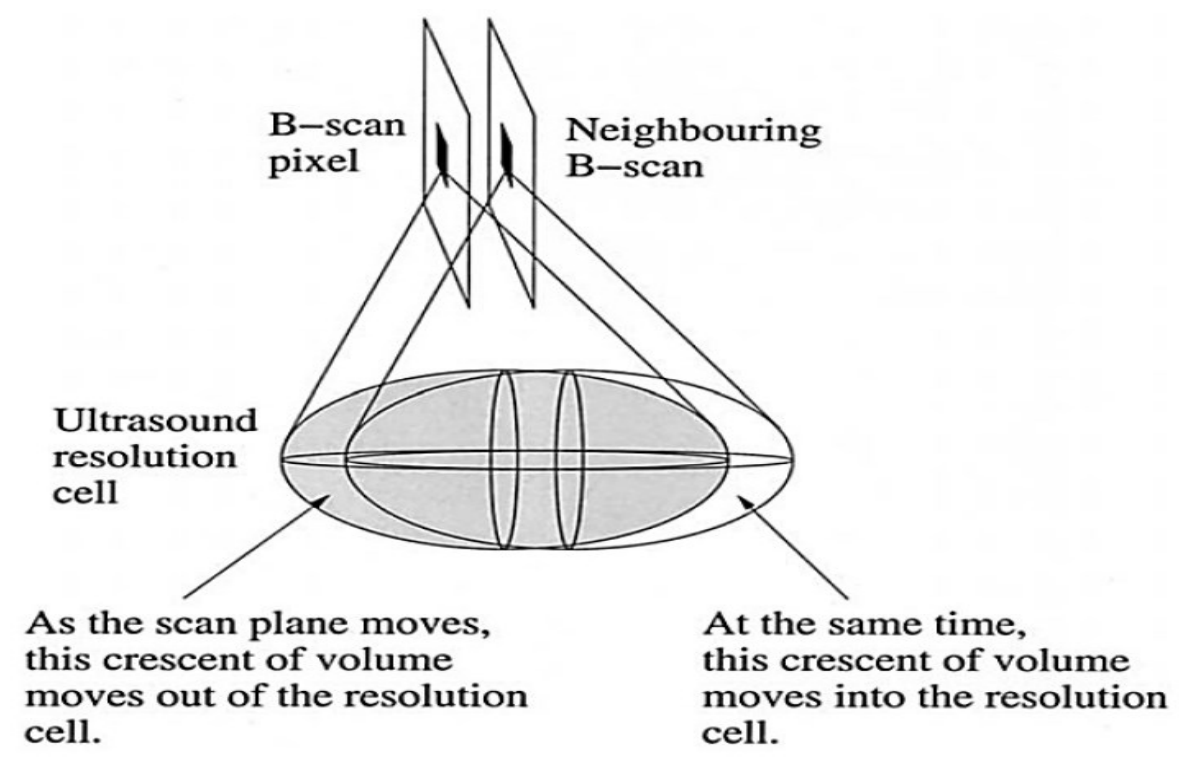

Figure 3-8: Imperfect elevation focus of the ultrasound probe from [16].

The concept of the speckle decorrelation curve introduced by Gee et al. and Afsham et al. [16], [41] only considers Fully Developed Speckle (FDS) while scanning the meat phantoms. However, Rivaz et al. [92] mention that real tissue such as vessel walls rarely contain FDS. Consequently, the elevation distance is underestimated from that of the conventional correlation algorithm, which degrades the accuracy of the motion estimation realized in the real tissue. To solve this, Tuthill et al. [20] developed an automatic algorithm for detecting FDS regions in the images to estimate the elevation distance. Prager et al. and Hassenpflug et al. [47], [93] applied three non-collinear pairs of patches 
to estimate the out-of-plane in six DOF between the two planes. However, the accuracy for estimating the out-of-plane motion can still be improved.

To do so, researchers considered both the effects of the FDS and non-FDS statistical distributions in speckle patterns simultaneously. Gee, et al. [16] introduced a technique that measures the amount of coherency at a given point in the B-mode US images by examining both the axial and lateral correlation curves in instead of acquiring the elevation specifications from the manufacturer of the ultrasound probe. This technique is also known as an adapted decorrelation curve as it accounts for the effects of coherent scattering.

Afsham et al. [40], [41] introduced the Rician-Inverse-Gaussian model for separating the coherent and non-coherent effects inside the patches of US images in real tissues. However, this model is based on the radio frequency (RF) ultrasound signal as mentioned in Section 2.7.

Both Bahramabadi and Gao et al. [15], [27] utilize the ultrasound B-mode image registration instead of relying on the RF ultrasound signal for determining the correlation coefficient. The ultrasound B-mode image registration overcomes the limitation of RF availability during the clinical application. However, there are large standard deviations in both articles, which lead to large marginal errors.

\subsubsection{Regression Based Method}

Bahramabadi [27] mentions that the regression-based method is similar to the correlationbased method. It applies the best linear estimation of the circular Gaussian distributed RF associated with the FDS patterns. This approach relates to the correlation of the speckle patches.

\subsubsection{Learning Based Method}

Laporte et al. [94] introduced a the relatively new learning base method based on using US data set images from virtual phantoms. By applying this data set as a training data for learning its relationship with respect to the in-plane statistics, a scale factor can be calculated for the nominal correlation curve. The elevation correlation length of the US signal can then be estimated in a specific media. 
Even though researchers employ a variety of strategies to improve the accuracy of the out-of-plane motion estimation, there are still constraints and limitations in clinical applications. Therefore, it is still an ongoing research topic for the freehand 3D ultrasound system.

\subsection{Challenges in the 3D Freehand Ultrasound System}

There are two significant challenges for the 3D freehand ultrasound system. Bahramabadi [27] notes a requirement for attaching the extra position sensors to the ultrasound probe, though various trackers are already available. The freehand ultrasound system is not widely accepted in clinical usage due to a lengthy and complicated calibration process. When operating the optical sensors, sonographers must ensure a clear sight line between the cameras and the optical sensors attached on the probe. When operating the electromagnetic sensor attached on the probe, sonographers must ensure there are no metallic objects nearby and that the sensor is within the operational region.

In addition, errors can occur during the process of synchronization between the ultrasound imaging and data from the position sensor due to unknown latencies that occurs during its operation, negative affecting the analysis.

Furthermore, implementing the complicated reconstruction algorithm in the visualization system increases computation time. If the simple algorithm is implemented, the accuracy cannot be maintained due to the real-time constraint. As a result, sonographers must currently choose between accuracy and computation time. The final goal of the 3D freehand ultrasound system is to find the algorithm that will balance both the accuracy and computation time. 


\section{Chapter 4: Methodology}

\subsection{Pre-Experiment Setup}

Spatial, speckle, and meat phantoms were prepared to simulate the properties of real tissues such as the speed of propagation, scatters, attenuation effect, elasticity, and scatters as the in-vitro study.

- The spatial phantom mimics the tissue properties with crossed nylon wires during the spatial calibration process as illustrated in Figure 4-1. As mentioned in Section 3.3.2, the spatial calibration locates each pixel of the 2D B-mode image into the 3D space so that rows (u) and columns (v) of images can be obtained in each B-scan. Detailed procedures for making the spatial phantom are listed in Appendix C.

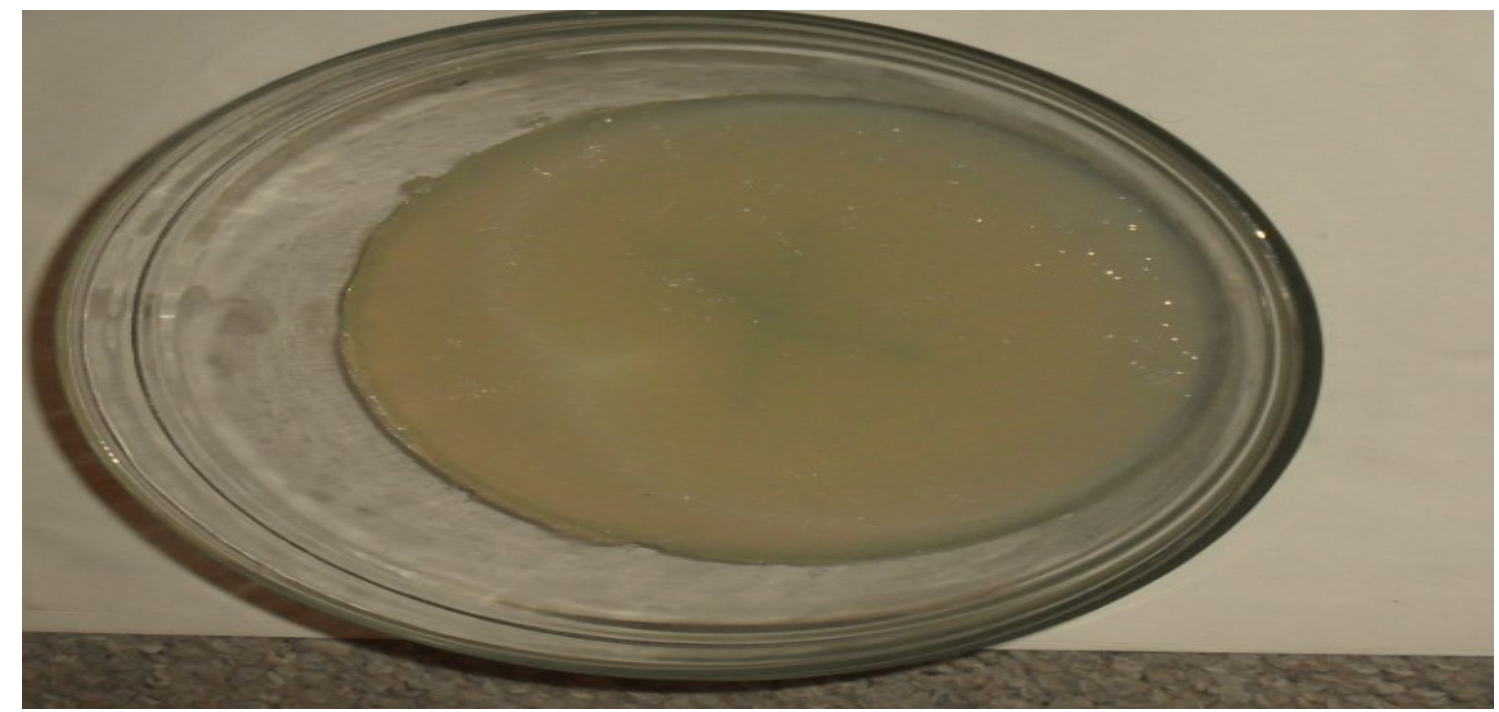

Figure 4-1: Spatial phantom 
- The speckle phantom was shown on the left of Figure 4-2. Its purpose was to simulate the Rayleigh scattering in real tissues. Graphite powders are applied in the speckle phantom to present the scatters distributed randomly. Detailed procedures for making the speckle phantom were listed in Appendix D. The acquired B-mode image for speckle phantom was displayed on the right of Figure 4-2.

- Meat phantoms were used for the elevation distance estimation. As illustrated in Figure 4-3, beef and chicken were prepared to simulate the coherent scattering in the real tissue as to create the adaptive decorrelation curve. The dimeson of chicken, beef and eraser was $0.8 \mathrm{~cm} \times 1.3 \mathrm{~cm}, 0.8 \mathrm{~cm} \times 2 \mathrm{~cm}$ and 0.5 $\times 2 \mathrm{~cm}$. Detailed procedures for making the meat phantoms were listed in Appendix E.
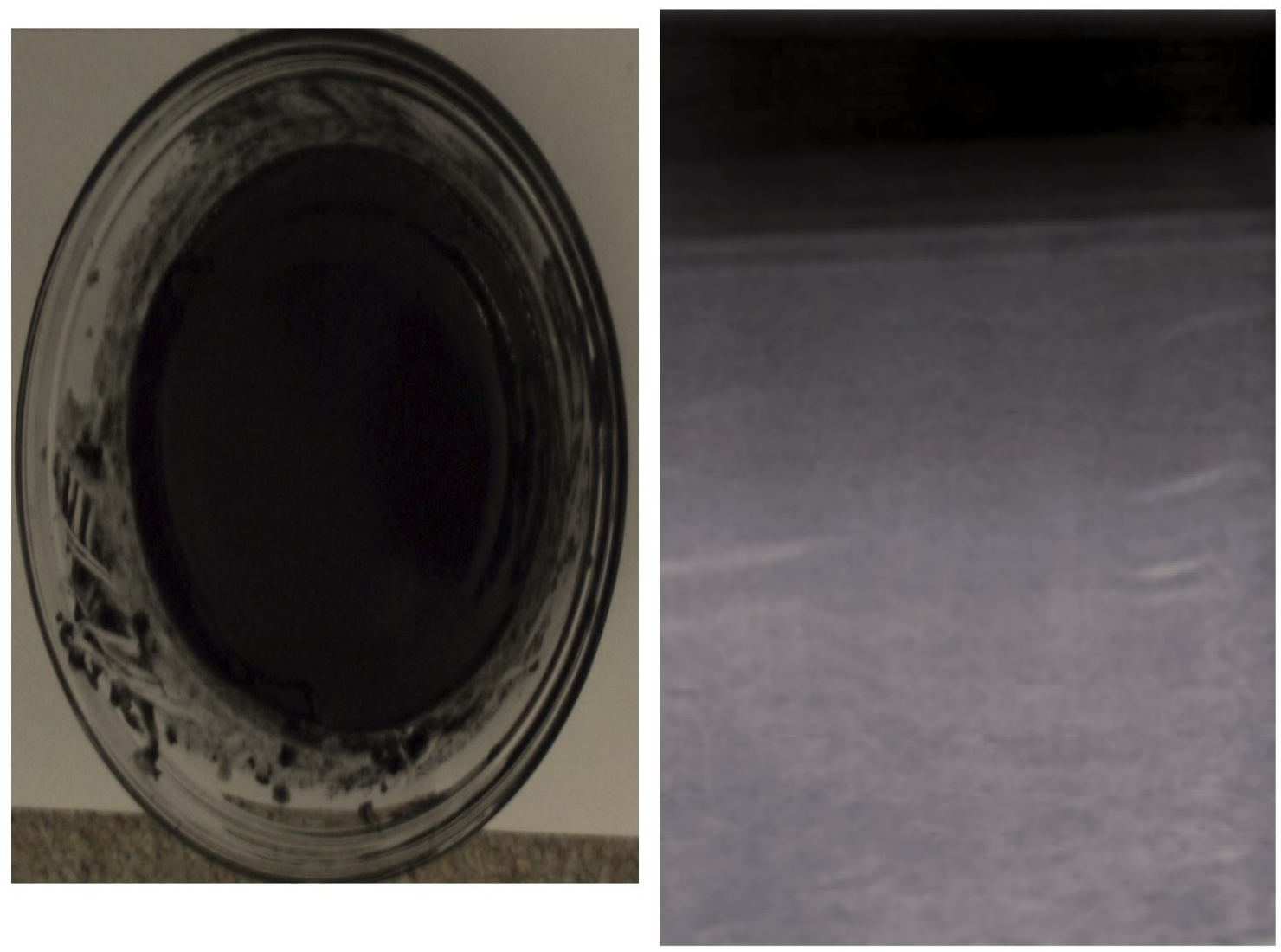

Figure 4-2: Speckle phantom (left). The Raleigh scattering simulated from graphite powders in Bscan of Speckle phantom (right) 


\subsection{Experimental Setup for Medical Ultrasound Image System and the Probe}

The equipment employed in this experiment included the medical ultrasound imaging system from Ultrasonix SonixTablet (Ultrasonix Ltd., Canada) [95] and an EM sensor from PATRIOT, Polhemus [96]. A more detailed account of the experimental setup follows.

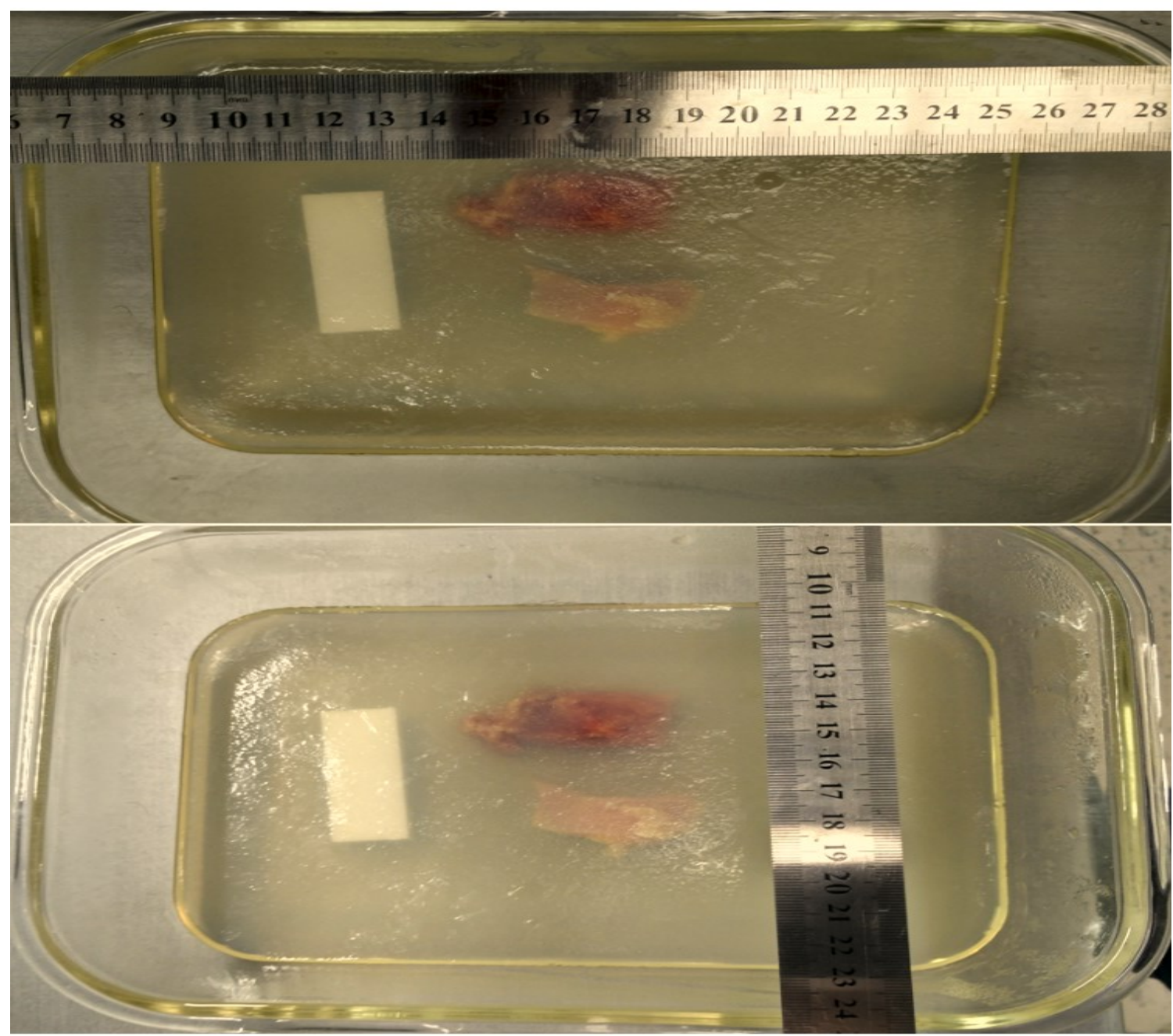

Figure 4-3: The size of chicken, beef and eraser in meat phantoms for the elevation distance estimation

- The medical ultrasound image system is powered by a Windows-Embedded operating system with built-in frame grabbing cards. During the data acquisition process, the system captures two-dimensional B-mode images in real-time. The 
ultrasound machine is displayed in Figure 4-4.The SonixDVR and Cine Loop software packages recorded the video clips as according to the manual of SonixTablet [95]. Cine Loop was chosen here since it can provide various frame rates with a maximum recording time of 30 seconds. The steps for setting up the Cine Loop properly are illustrated in Appendix E.

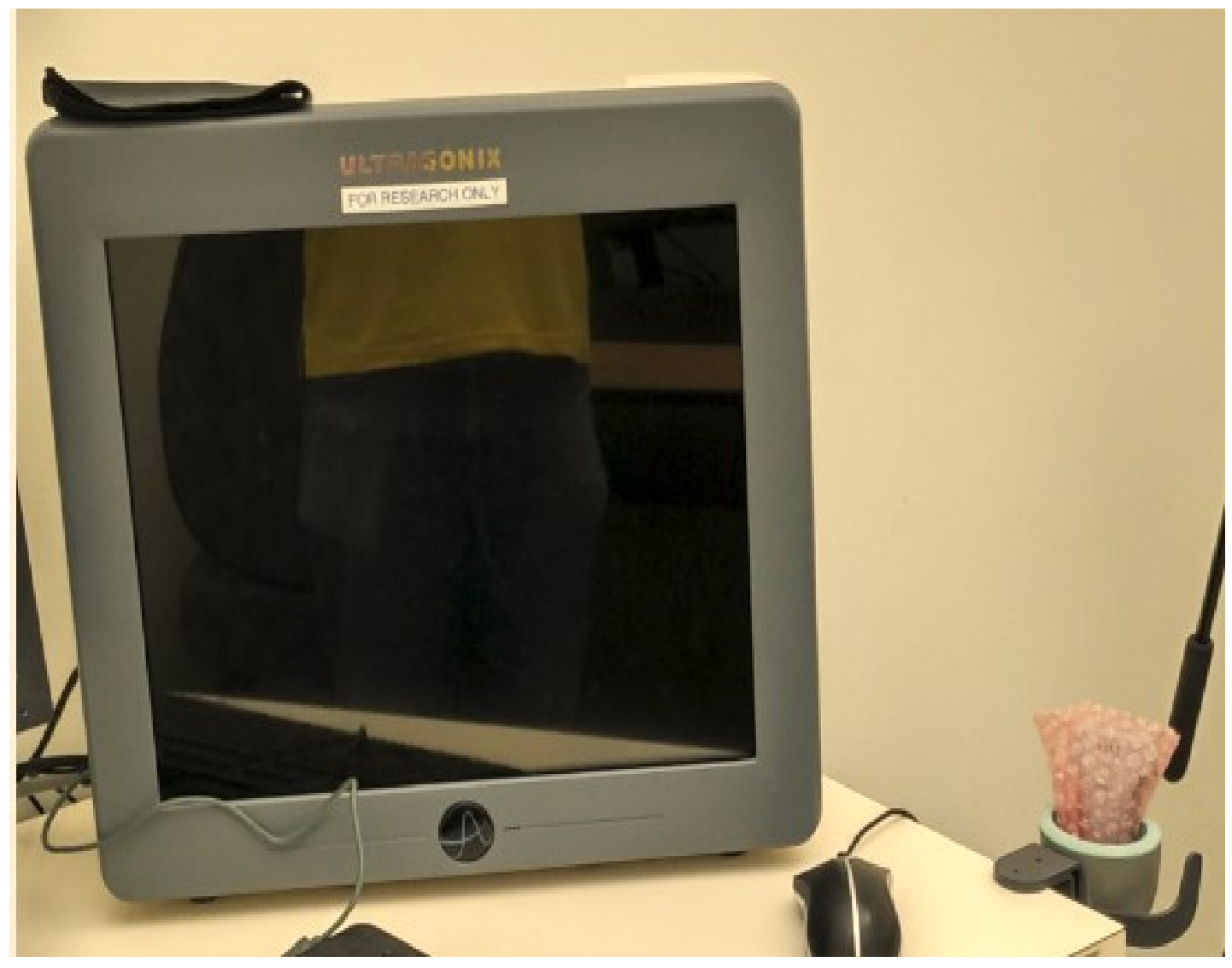

Figure 4-4: Ultrasonix SonixTablet ultrasound image system

- The linear ultrasound probe with model number "L14-5/38" as described by WikiSonix's website [97] appears in Figure 4-5. It is a $39 \mathrm{~mm}$ linear array transducer with frequency in the range of $5 \mathrm{MHz}$ to $14 \mathrm{MHz}$ with depth from $2 \mathrm{~cm}$ to $9 \mathrm{~cm}$. The probe frequency is at $10 \mathrm{MHz}$ with $3 \mathrm{~cm}$ in depth for maintaining the same frame rate at 60 frames per second (fps) as the Patriot sensor. There are 128 individual piezoelectric crystal transducer elements with a separation distance of 
$305 \mu$ s. The reflective echo is processed in SonixTablet with a 14-bit analog to digital converter (ADC).

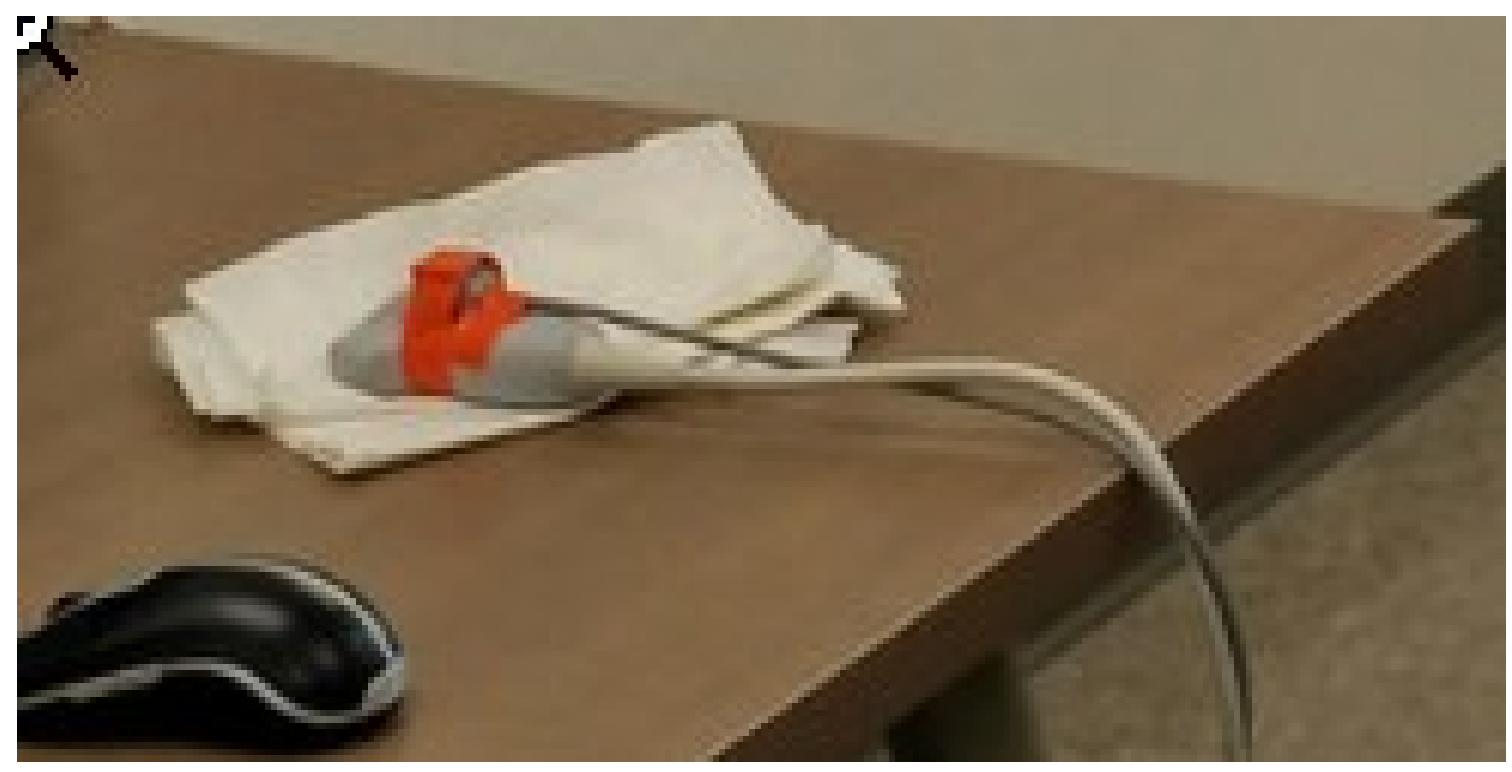

Figure 4-5: Sensor is attached on the ultrasound probe bound with electrical tape to detect EM field

\subsection{Electrical Magnetic Sensor Set Up}

Bahramabadi [27] chose the EM sensor due to the balance of cost and accuracy for calibrating the decorrelation curve in the 3D freehand ultrasound system. The PATRIOT electromagnetic sensor used here consists of a sensor (receiver), source (transmitter), system electronic unit and power supply as illustrated in Figures 4-5 and 4-6. The sensor is mounted on top of the probe as illustrated in Figure 4-5.

The system electronic unit contains the hardware and software for configuring the system for generating the signal to the source (transmitter) to produce an EM field. Once the sensor (receiver) receives the EM field, it sends the signal back to the system unit for processing, as stated in the PATRIOT's user manual [98]. The field source (transmitter) is 250 grams. The sensor (receiver) is 23 grams. The sensor (receiver) and source (transmitter) are placed in an environment with less metal present to reduce signal distortion. 


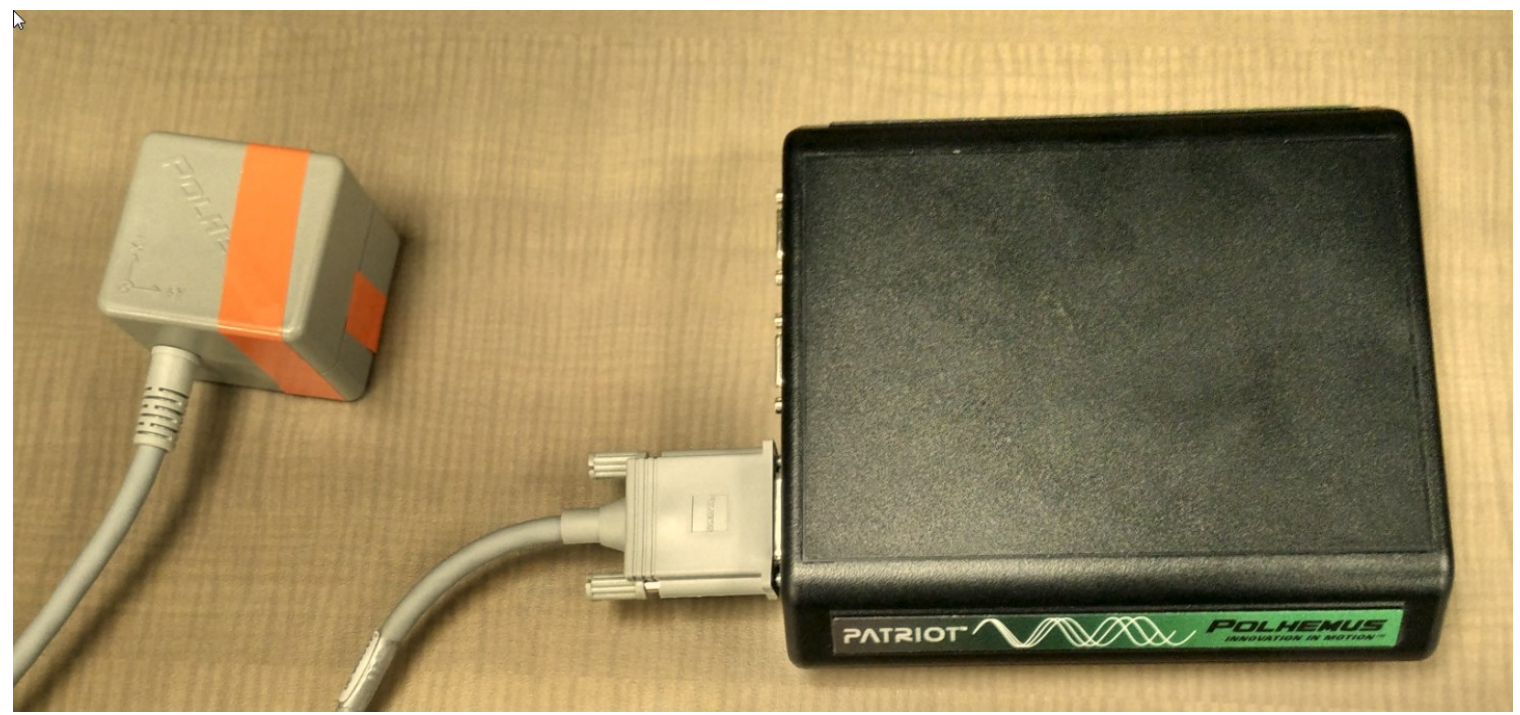

Figure 4-6: The EM source (left) and system electronic unit (right) in the Patriot sensor for producing the EM field

The Patriot sensor can provide the six-degrees-of-freedom (DOF) data acquisition in real-time while the probe moves in space. The system has its built-in PiMgr software for displaying the resulted data on the screen and recording the data including positions of $\mathrm{X}, \mathrm{Y}$ and $\mathrm{Z}$ with orientations of azimuth $(\alpha)$, elevation $(\beta)$ and roll $(\gamma)$ in a text file.

The root means square (RMS) of the system is $1.542 \mathrm{~mm}$ RMS for the $\mathrm{X}, \mathrm{Y}$ and $\mathrm{Z}$ positions with 0.4 degrees RMS in the sensor orientation for the static accuracy, as mentioned in the PATRIOT's user manual [98]. This is under an ideal condition where it is in a non-metallic environment and the distance between the sensor (receiver) and source (transmitter) is at most $75 \mathrm{~cm}$.

If the operating distance exceeds this range, the performance accuracy may degrade. The latency is within $18.5 \mathrm{~ms}$ with the frame updating rate at $60 \mathrm{~Hz}$ for each sensor. The system is configured in inches for the Cartesian coordinates. The orientation is presented in the Euler angles.

\subsection{Data Acquisition}

There were three types of data acquisition on the spatial phantom, speckle phantom and meat phantoms in our study. Figure 4-7 demonstrated an overview of the data acquisition. 


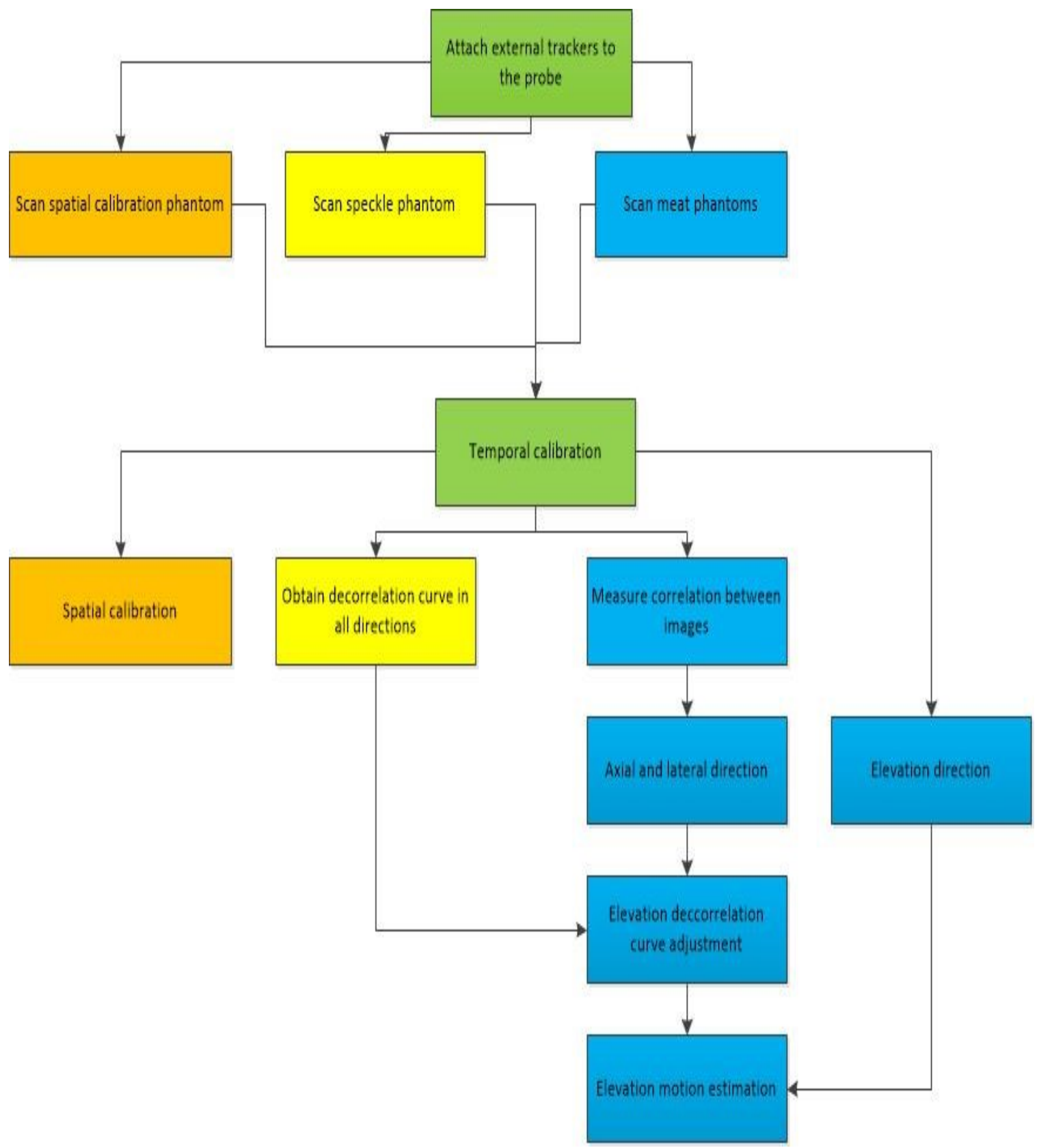

Figure 4-7: An overview of data acquisition and data analysis for the elevation distance estimation reconstructed from [27]

After preparing the phantoms and setting up the devices including the ultrasound machine and PATRIOT sensor, the next step would be to collect the B-scans and positions with their corresponding orientations. The first step corresponding to Figure 4-7 was to attach the sensor to the probe by electrical tape as displayed in Figure 4-5. 


\subsubsection{Data Acquisition with the Spatial Phantom}

The spatial phantom consisting of two nylon cross-wires was scanned with the spatial calibration. Bahramabadi and Prager et al. [27], [54] explain that the purpose of the spatial calibration is to obtain the precise location of the pixel in the image in rows $(\mathrm{u})$ and column (v) with respect to six other positions from the PATRIOT sensor attached to the probe.

Data consistency in our results and clinical applicability are concerns, especially as Pagoulatos et al. [58] describe the spatial calibration time as approximately 2 minutes. The decrease in data consistency is known to occur as Kim et al. [14] have observed that properties of the phantom such as elasticity degrade over a period of time. Therefore the acquired data could give different results according to these changes. In clinical settings, it is impractical for patients to wait for sonographers to perform spatial calibrations.

The temporal calibration was initialized at the beginning of every scan for all phantoms. As a result, synchronization can occur between frames captured in the internal ultrasound machine and positions are recorded in the PATRIOT sensor.

The following section listed our improved procedure on calibration sequence of events during the study reducing the spatial calibration time from 2 minutes to 10 seconds from following step 1 to step 4. It could be verified with the recording time in the video clip.

1) Moved the probe closer to the pre-crossing section of the two nylon wires

2) The $1^{\text {st }}$ temporal calibration started ( $1^{\text {st }}$ spike).

3) Clicked " 1 " to record on the screen of ultrasound machine.

- Ensured "14" in the label of ultrasound probe "L14-5" aligning in the middle of the cross section of nylon wire.

- $\quad$ Swang the probe right before hitting the $1^{\text {st }}$ cross point.

- $\quad$ Continued swing quickly after hitting the crossing point.

- $\quad$ Swang the probe back and stop before hitting the $2^{\text {nd }}$ cross point.

- $\quad$ Rotated the probe quickly either in the clockwise or counter clockwise direction before hitting the $3^{\text {rd }}$ cross point.

4) Kept the probe moving until hearing a "beep" sound from the ultrasound machine. It meant the recording has ended.

5) Did the $2^{\text {nd }}$ temporal calibration again immediately ( $2^{\text {nd }}$ spike). 


\subsubsection{Data Acquisition with the Speckle Phantom}

After scanning the spatial phantom with the temporal and spatial calibrations, Prager et al., Mercier and Hsu et al. [55]-[57] mention that we needed to scan the speckle phantom to obtain images of FDS with the known elevation separation distances in axial, lateral and elevation directions. The temporal calibration also occurred at the beginning as well. The following section outlined the procedures in this thesis to acquire data in the speckle phantom.

1) Moved the probe to one edge of the bow.

2) Clicked "1" to record on the screen of ultrasound machine.

3) The temporal calibration started ( $1^{\text {st }}$ spike $)$.

4) Moved the probe to the other end of the phantom.

5) Kept the probe moving until hearing a "beep" sound from the ultrasound machine. It meant the recording has ended.

The speckle phantom results were first used to measure the correlation coefficients by dividing the images into patches between frames. From here, matching patches could occur in the image with a specific separation distance and correlation value. The correlation curve could be fitted with the sets of correlation coefficients for each patch. Finally, the decorrelation curve was constructed for each specific patch from the calculated correlation coefficient with respect to its axial, lateral and elevation distances in the given ultrasound transducer.

\subsubsection{Data Acquisition with the Meat Phantoms}

The meat phantoms were scanned to form the adaptive decorrelation curve for estimating the elevation distance between B-scan images in real tissues, which in this experiment consist of beef and chicken. The temporal calibration also took place at the beginning of the scan. The following procedure collects data in the meat phantoms:

1) Moved the probe to the edge of the eraser corresponding to the chicken or beef phantom.

2) Clicked " 1 " to record on the screen of ultrasound machine. 
3) The temporal calibration started (1st spike).

4) Moved the probe to the other end of the phantom.

5) Kept the probe moving until hearing a "beep" sound from the ultrasound machine. It meant the recording has ended.

\subsection{Data Pre-Processing}

During data acquisition, the ultrasound system captured a complete view of the B-scan image into video clips so we selected the ROI by cropping the image.

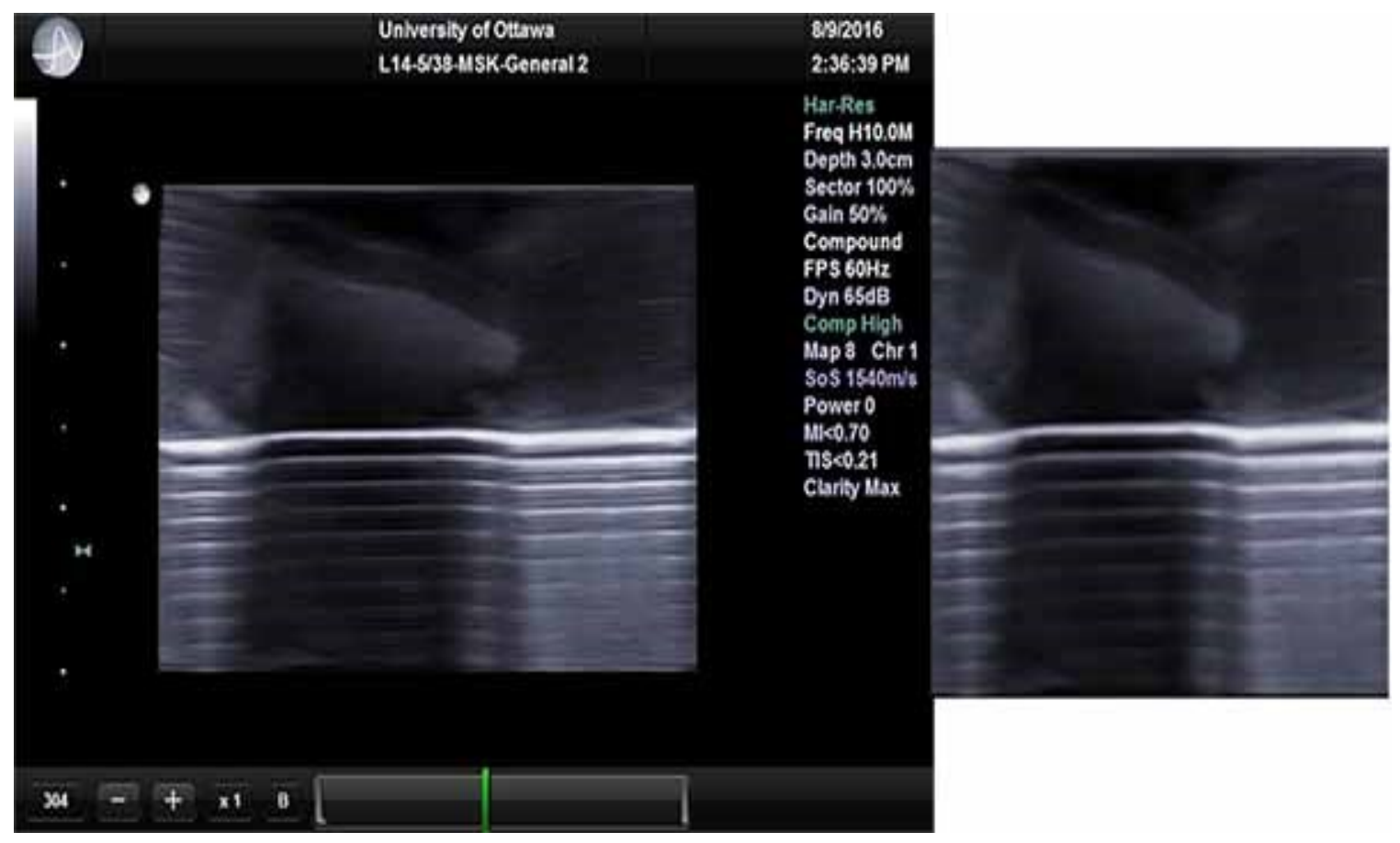

Figure 4-8: Cropping the image in the beef phantom

At the same time, each image was required to be extracted from the video clips for data analysis. The video clips were edited with Adobe Premier CS 2015, and were 395 pixels wide and 400 pixels high. The smallest axial and lateral displacements with respect to the B mode image were $0.075 \mathrm{~mm} /$ pixel as $30 \mathrm{~mm} / 400$ pixels since the axial depth was $30 \mathrm{~mm}$. The size of the image was $2.9625 \mathrm{~cm}\left(0.075[\mathrm{~mm} / \mathrm{pixel}] \times 395[\right.$ pixels] $) \times 10^{-1}$ [cm/mm]) in width and $3 \mathrm{~cm}\left(0.075[\mathrm{~mm} /\right.$ pixel] $\mathrm{x} 400$ [pixels] $\left.) \times 10^{-1}[\mathrm{~cm} / \mathrm{mm}]\right)$ in depth as shown in Figure 4-8. The cropping and extraction were applied for all image data then the data preprocessing was performed later. 


\subsubsection{Data Preprocessing in Temporal Calibration}

As mentioned in Section 3.3.1, the temporal calibration process involves matching the ultrasound images with their corresponding positions due to latencies in both the ultrasound machine and position sensing device. It requires synchronization between them by adjusting their time stamps.

The Stradx freehand 3D system introduced by Nelson et al. [84] is implemented in this thesis. It requires providing step inputs to the imaging and positioning streams by holding the probe for few seconds then moving away from the skin quickly. The data is thus in position, and the images are recorded simultaneously. The difference in timing between the two streams during this sudden change can then be used to adjust the offset in position sensor time stamp.

To determine the step input, the position data is analyzed by measuring the maximum amount of changes between frames in the sequence. At this stage, the orientation data is ignored. The probe should be held steady for 2 to 3 seconds for each acquisition of data, meaning each successive reading should be within $1 \mathrm{~mm}$ of the last. When the probe was moved away suddenly, there was a spike in the position data at frame 110 as in Figures 49 and 4-10. There is a damp instead of a spike due to a matching of the coordinate system as the source contains electromagnetic emitter.

The two frames are averaged if their difference is greater than $1 \mathrm{~mm}$. Upon obtaining the exact time in both positioning and imaging streams where the step input takes place, Bahramabadi, Hsu et al. and Prager et al. [27], [54], [67] state that the time adjustment between the two streams can be done. Since the frame rate in the positioning stream is equivalent to the frame rate in the imaging stream, no interpolation is needed. The most recent image can be matched with the most recent position. Both the PATRIOT sensor and ultrasound imaging system were at 60 frames per second, which is shown in Figure 4-11. 


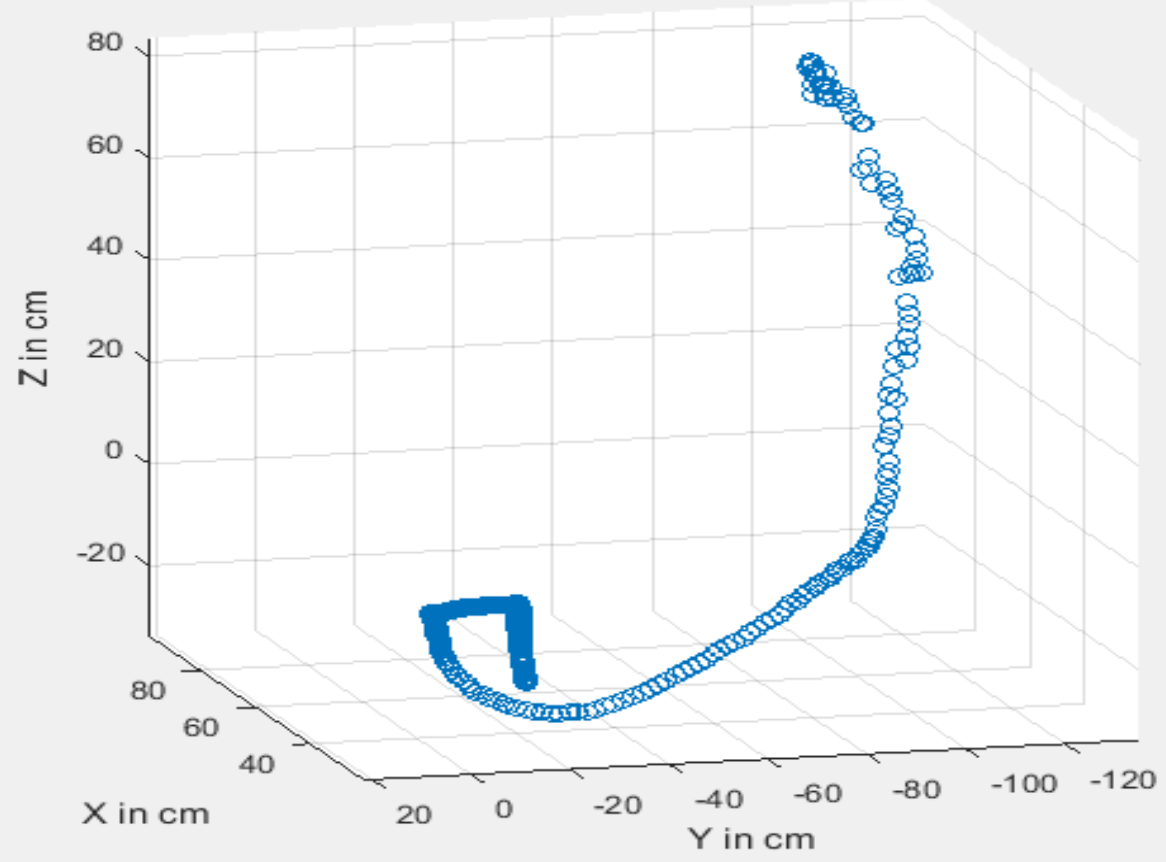

Figure 4-9: Temporal calibration in beef phantom in 3D from raw data from EM sensor attached to the probe

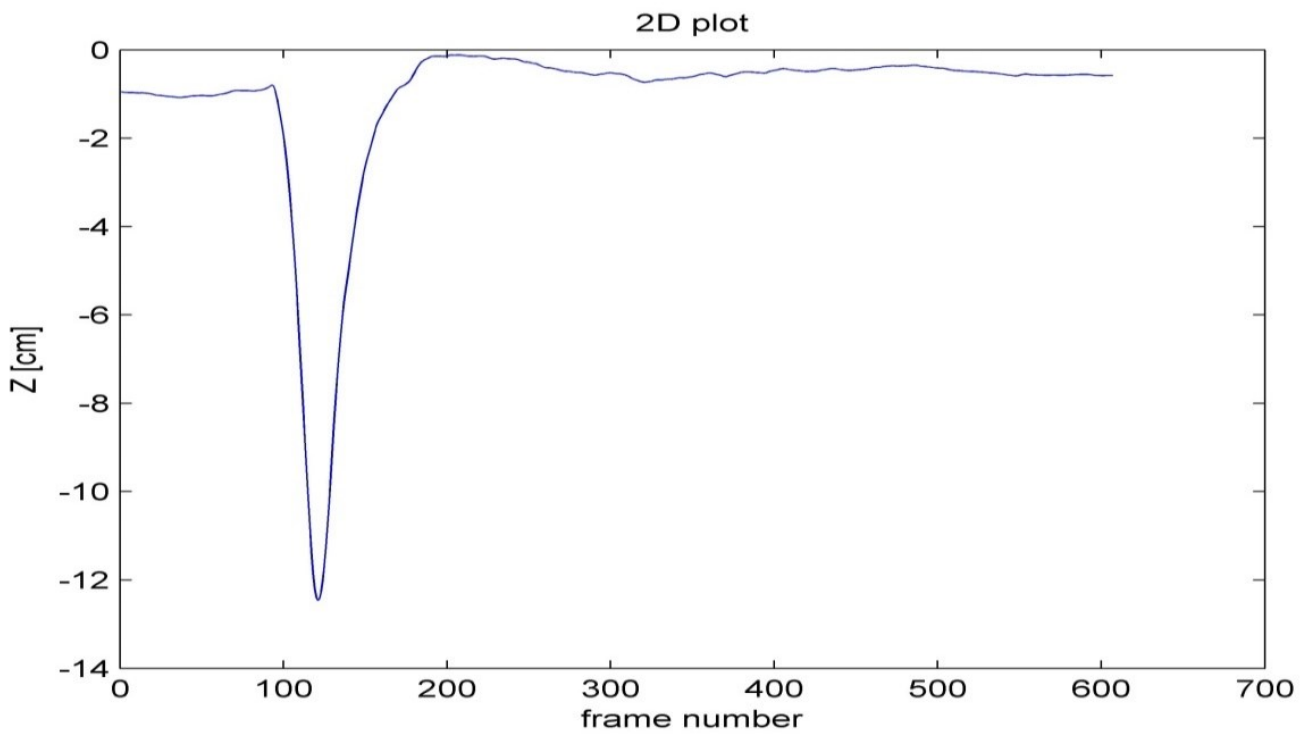

Figure 4-10: Temporal calibration in beef phantom in the direction of $\mathrm{Z}$ axis with respect to frame from raw data 


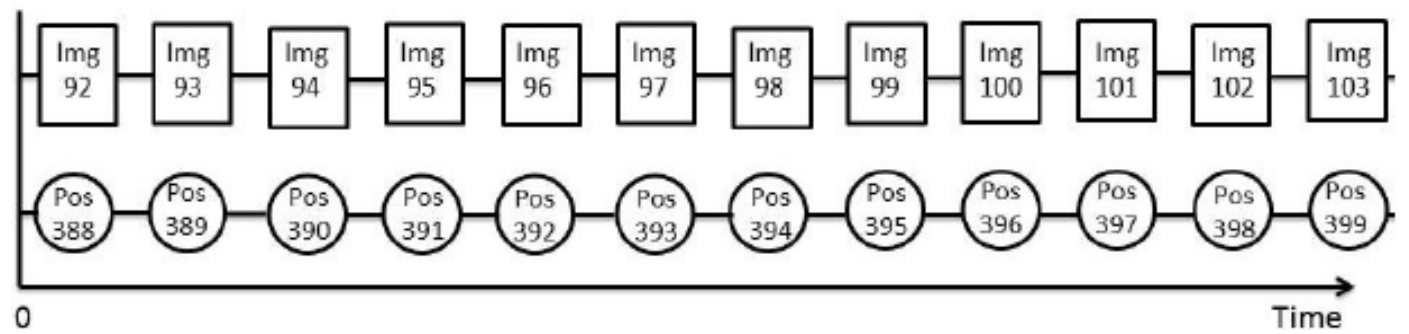

Figure 4-11:Synchronization of the Patriot sensor with the ultrasound imaging system at the same frame rate from [27]

\subsubsection{Data Pre-processing in Spatial Calibration}

After the synchronization between the imaging and positioning streams is completed in the temporal calibration, the exact position of each image pixel in the 3D space from spatial calibration needs to be determined.

The position sensor acquires the 3D location of the small sensor attached to the ultrasound probe. It is necessary to obtain the location of the scan plane within each pixel of the image in the 3D space. The purpose of the spatial calibration is to estimate the six degrees of freedom (DOF) rigid body transformation matrix between the position sensor coordinate system and image plane coordinate system.

Prager et al. [57] list three steps for obtaining the location of the pixels in 3D space as illustrated in Figure 4-12 since the output of the EM position sensor is the coordinate of the receiver with respect to the transmitter. Firstly, the pixels need to be transformed from the coordinate system of the image plane indicated as $\mathrm{P}$ into the coordinate system of the receiver or sensor marked as R. They then must be transformed from the coordinate system of the receiver or sensor marked as $\mathrm{R}$ to the coordinate system of the transmitter or source marked as T. Finally, they must be transformed from the coordinate system of the transmitter or source marked as $\mathrm{T}$ to the coordinate system of the volume reconstruction marked as $\mathrm{C}$. The overall calibration equation is listed as Equation 4.6, and obtains the location of each pixel from the image in the 3D space of the volume coordinate through multiplication of transformation matrices. 

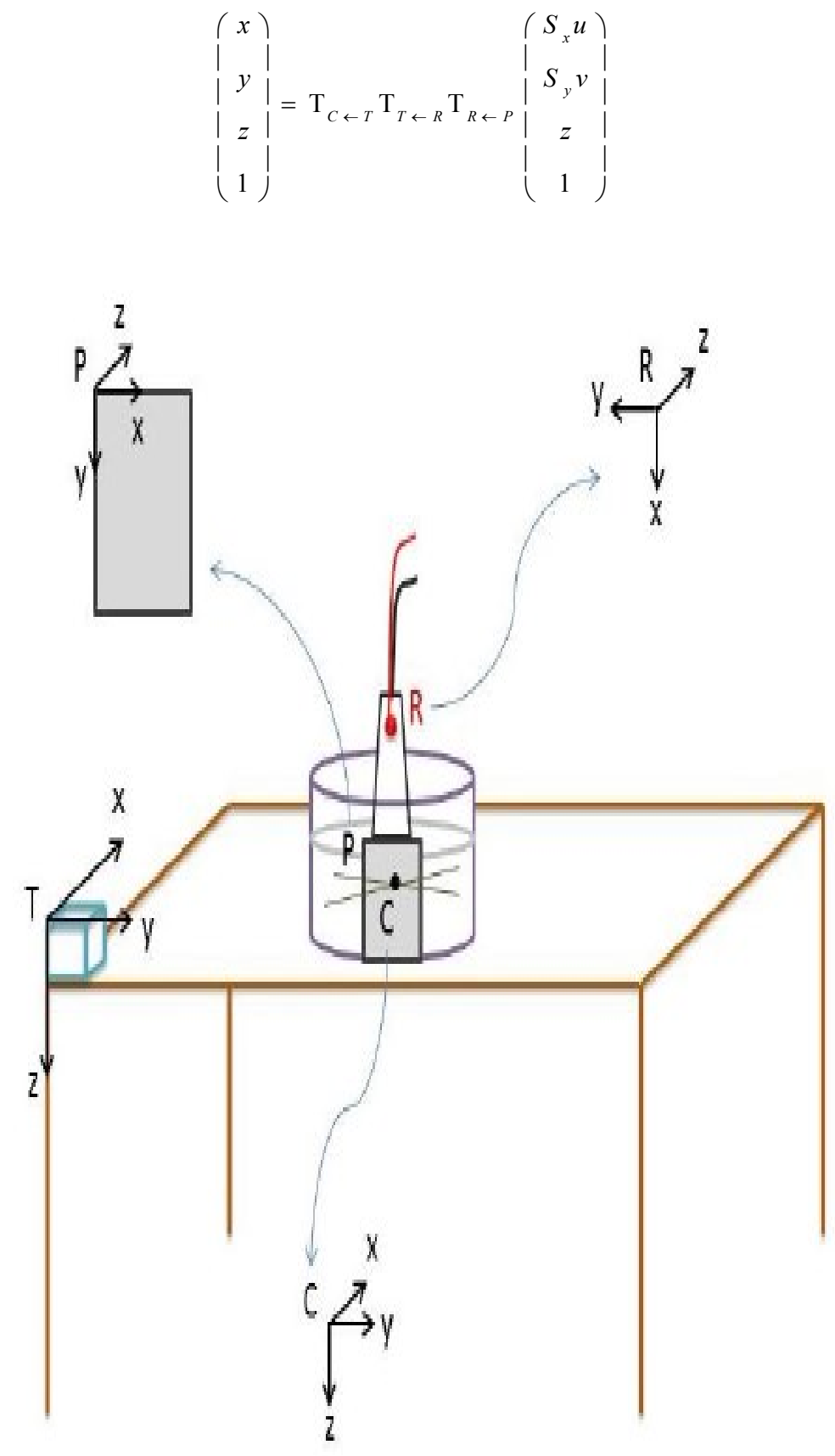

Figure 4-12: Different coordinate systems for image plane (P), receiver/sensor $(R)$, transmitter/source (T) and volume reconstruction (C) from [27]

Within the Equation 4.7, $\mathrm{T}_{\mathrm{i} \leftarrow \mathrm{j}}$ represents the transformation from the coordinate system of $i$ to the coordinate system of $j$. Prager et al. [57] indicate that the values of $u$ and $\mathrm{v}$ represent the column and row of the image plane at a specific frame during the data acquisition process in the spatial phantom. Sx and Sy are the scale factors in the unit of 
milometer over pixel ( $\mathrm{mm} / \mathrm{pixel})$ and related to the image positions of $u$ and $v$ while reaching the center of the crossed nylon wires.

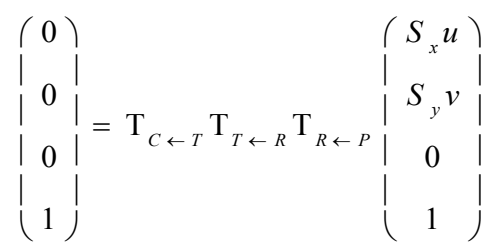

Only two calibration processes were presented as the cross-wire in the speckle phantom for our study. First, the origin of reconstruction volume indicated as $\mathrm{C}$ is at the intersection of the wire at time t1. Second, the same point was imaged in various probe directions within 10 seconds from time t1 to time t10. Due to assigning the location of the volume reconstruction at the center of the cross-wire, it led to a change from Equation 4.6 to Equation 4.7.

The value of unknowns as $T_{C \leftarrow T}$ and $T_{R \leftarrow P}$ with the known values including $u, v, S_{x}$ and $\mathrm{S}_{\mathrm{y}}$ and $\mathrm{T}_{\mathrm{T} \leftarrow \mathrm{R}}$ in Equation 4.7 give three equations with the first three rows in Equation 4.6. The data from the electromagnetic sensors allow the final value of $T_{T \leftarrow R}$ to be obtained from its position and orientation within its respective frame. The six degree of freedom (DOF) transformation has six variables with positions in $\mathrm{x}, \mathrm{y}$ and $\mathrm{z}$ and orientations in $\alpha, \beta$ and $\gamma$. The electromagnet sensor (Patriot, Polhemus, Colchester, Vermont) implements the Z-Y-X Euler angles coordinate system. The uppercases of X, Y and $\mathrm{Z}$ represent the state of reference at zero orientation, and the lowercases of $\mathrm{x}, \mathrm{y}$ and $\mathrm{z}$ represent the current state of orientation at a specific frame from the sensor.

As illustrated in Figure 4-13, Euler angles consist of azimuth $(\alpha)$, elevation $(\beta)$ and roll $(\gamma)$ from the Patriot User Manual [98]. These angles represent the difference from the current states of orientation with respect to the zero states of orientation. It is also known as azimuth primary sequence. As rotation sequence starts from azimuth, elevation, and roll rotations, there are three steps. Firstly, the azimuth angle is about the rotation from axes of $\mathrm{X}$ to $\mathrm{X}^{\prime}$ and $\mathrm{Y}$ to $\mathrm{Y}^{\prime}$ around the $\mathrm{Z}$ axis. Secondly, the elevation angle is about the rotation from axes of $\mathrm{X}^{\prime}$ to $\mathrm{X}$ and $\mathrm{Z}$ to $\mathrm{Z}$ ' around $\mathrm{Y}^{\prime}$ axis. Thirdly, the roll angle is about the rotation of axes from $Z$ ' to $Z$, then another rotation occurs from $Y^{\prime}$ to $Y$ around the $X$ axis. 
Prager et al. [57] describe how the homogenous matrix for each transformation is listed in Equation 4.8 under Euler angles approach. As mentioned before, $\mathrm{u}$ and $\mathrm{v}$ represent the column and row indices of the pixel at the center of the crossed nylon wires. The value of $u$ and $v$ are assigned manually through detecting the point of intersection on the image as shown in Figure 3-3.

From our calibration method in Section 4.5.1, there were 169 data points within the duration of 10 seconds. The calibration calculation on spatial phantom only needed to be done once since the focus is shifted to the next stage in the speckle phantom for obtaining the fully developed speckle (FDS) decorrelation curve.

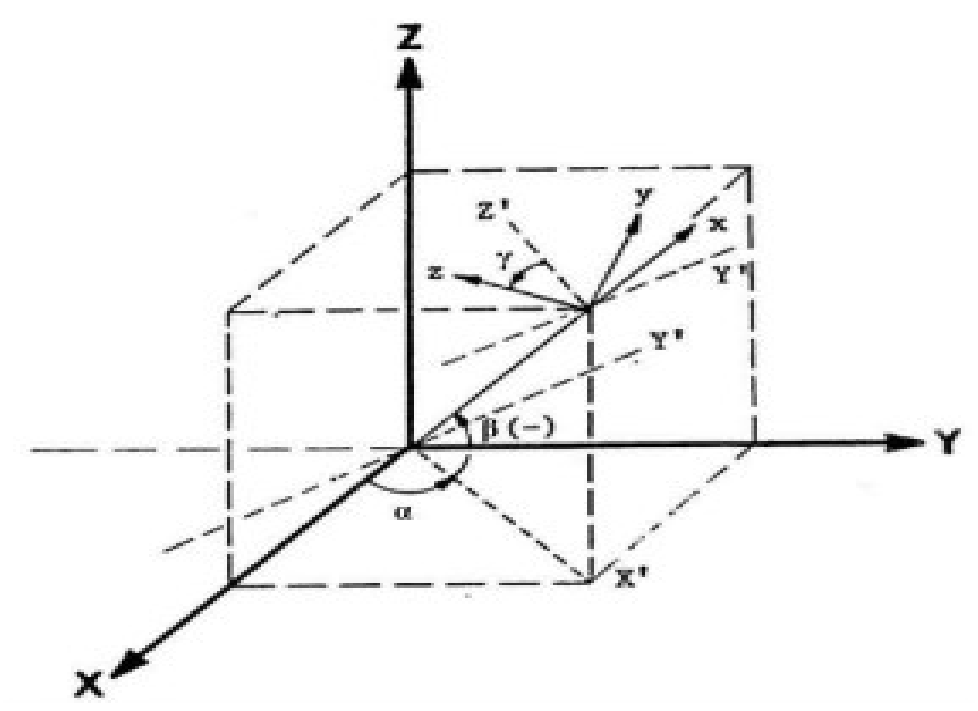

Figure 4-13: Euler angles in Z-Y-X for the Patriot sensor from [98]

The axial depth in this thesis was $3 \mathrm{~cm}$ with an image size of 400 pixels in height and 395 pixels in width. Therefore, the scale factor value for Sy was 30/400=0.075 mm/pixel. The scale factor for Sx was assumed to be $0.075 \mathrm{~mm} /$ pixel due to the symmetry of pixels in the square.

If there are m number of B-scans, Equation 4.7 will accumulate and generate a system of nonlinear homogeneous equation in the size of $3 \mathrm{~m}$ as shown in Equation 4.9. 


$$
\begin{aligned}
& \left(\begin{array}{llll}
\cos \alpha \cos \beta & \cos \alpha \sin \beta \sin \gamma-\sin \alpha \cos \gamma & \cos \alpha \sin \beta \sin \gamma+\sin \alpha \sin \gamma & x
\end{array}\right) \\
& \sin \alpha \cos \beta \quad \sin \alpha \sin \beta \sin \gamma+\cos \alpha \cos \gamma \quad \sin \alpha \sin \beta \cos \gamma-\cos \alpha \sin \gamma \quad y \\
& -\sin \beta \quad \cos \beta \sin \gamma \quad \cos \beta \cos \gamma \quad z \\
& \left.\begin{array}{llll}
0 & 0 & 0 & 1
\end{array}\right) \\
& 0=f(\theta, \phi)
\end{aligned}
$$

In Equation 4.9, the value of $\theta$ represents the measurement. The value of $\phi$ indicates the unknowns. Prager et al. [57] note that as long as there are more equations than unknowns, the system can be solved through the iterative method. As the LevenbergMarquardt algorithm is applied, some initial values are assigned to the elements within $\phi$ vector.

After multiple iterations, elements within the $\phi$ vector converge to a specific value and the $\Delta \phi$ is sufficiently small. The algorithm terminates once a successful iteration is obtained. Therefore, the convergent value of elements in the value $\phi$ becomes the answer to the non-linear homogeneous equation. It is important to understand that more than one solution could meet the condition of the equations. It is thus crucial to pick up the initial unknown value because the value selected can affect the value where $\phi$ converges.

Within this thesis, the estimations were done according to the experimental setup, meaning the data couldn't be perfectly accurate. After obtaining the values within the $\phi$ vector, the difference between the $3 \mathrm{D}$ locations of the pixel was at the center of cross section so the origin of volume reconstruction (C) could be obtained. The origin for the volume reconstruction represents the zero value in $\mathrm{X}, \mathrm{Y}$, and $\mathrm{Z}$.

From Figure 4-14 to Figure 4-16, the mean value represents the difference between the estimated and real origins of the volume reconstruction coordinate $(\mathrm{C})$. If the mean of the distribution is closer to the origin, the data is more accurate. In Figure 4-14, the mean value of the normal distribution is $2.6602 \mathrm{~mm}$ with a variance of $6.3813 \mathrm{~mm}^{2}$ in the $\mathrm{x}$ axis. In Figure 4-15, the mean value of the normal distribution is $1.7566 \mathrm{~mm}$ with a variance of $2.8611 \mathrm{~mm}^{2}$ in the y-axis. In Figure 4-16, the mean value of the normal distribution is $0.1718 \mathrm{~mm}$ with a variance of $0.0122 \mathrm{~mm}^{2}$ in the z-axis. 


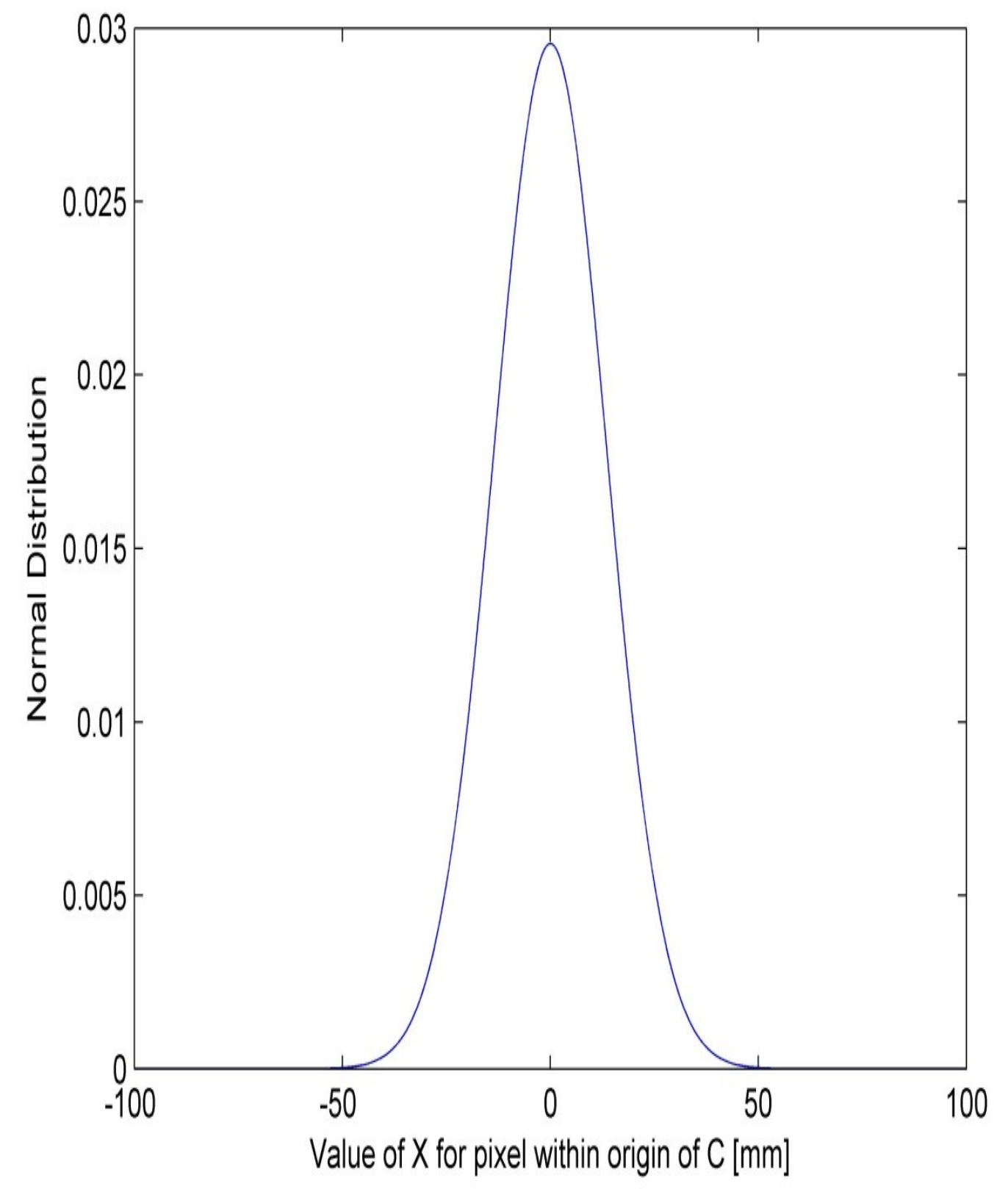

Figure 4-14: Normal distribution for values in $\mathrm{X}$-axis at the center location of volume reconstruction from various images in different directions. Variance is $6.3813 \mathrm{~mm}^{2}$. Mean is 2.6602 mm

The finest precision is $0.012 \mathrm{~mm}$ according to the Patriot User Manual [98]. The normal distributions from Figure 4-14 to Figure 4-16 show that the resolution cells are in the Gaussian shape according to the first order statistic in Section 2.7.1. Therefore, echo 
intensity can be expressed as the function of the elevation distance and resolution width in both Equations 2.8 and 2.9.

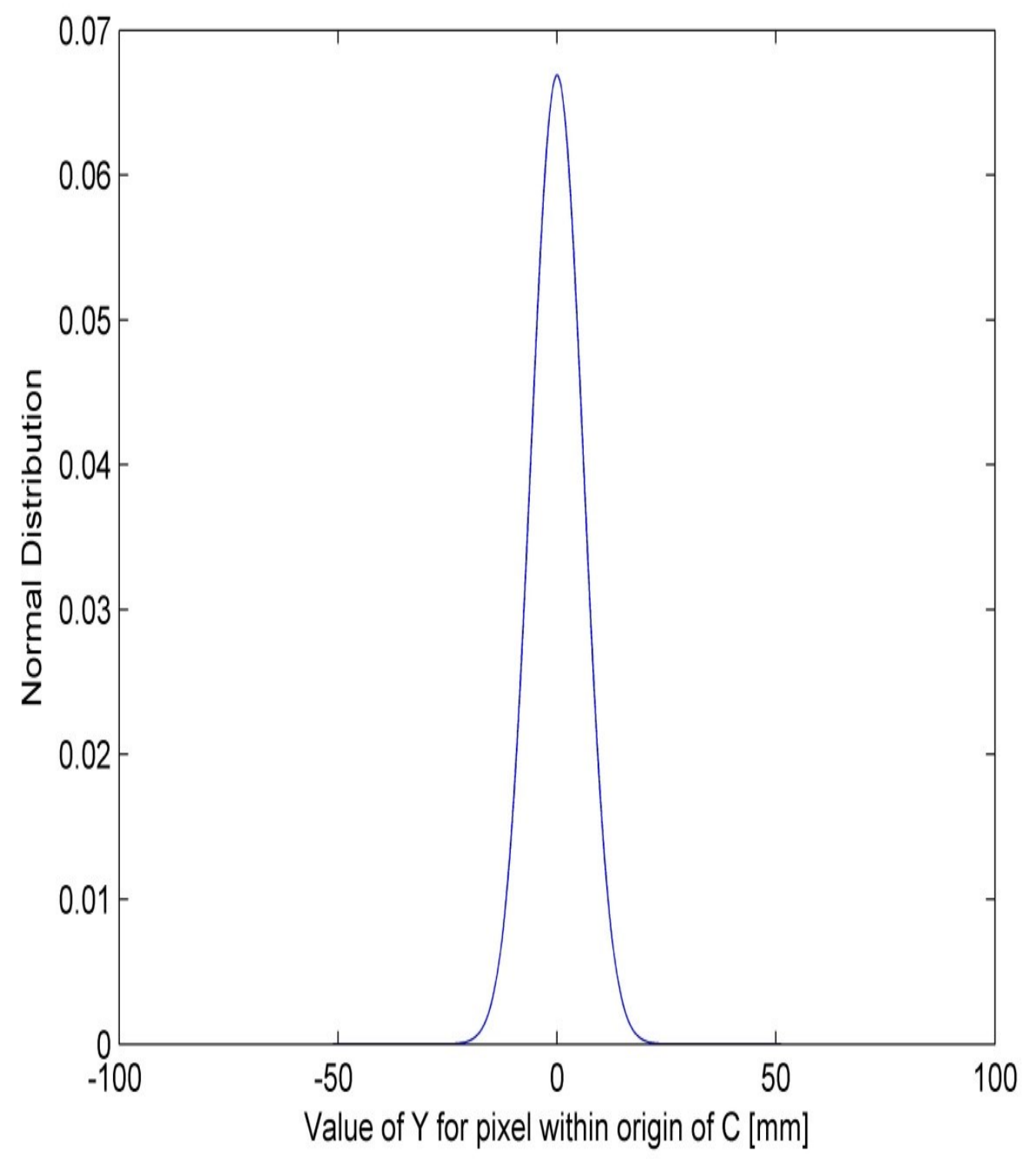

Figure 4-15: Normal distribution for values in $\mathrm{Y}$ axis at the center location of volume reconstruction from various images in different directions. Variance is $2.8611 \mathrm{~mm}^{2}$. Mean is $1.7566 \mathrm{~mm}$ 


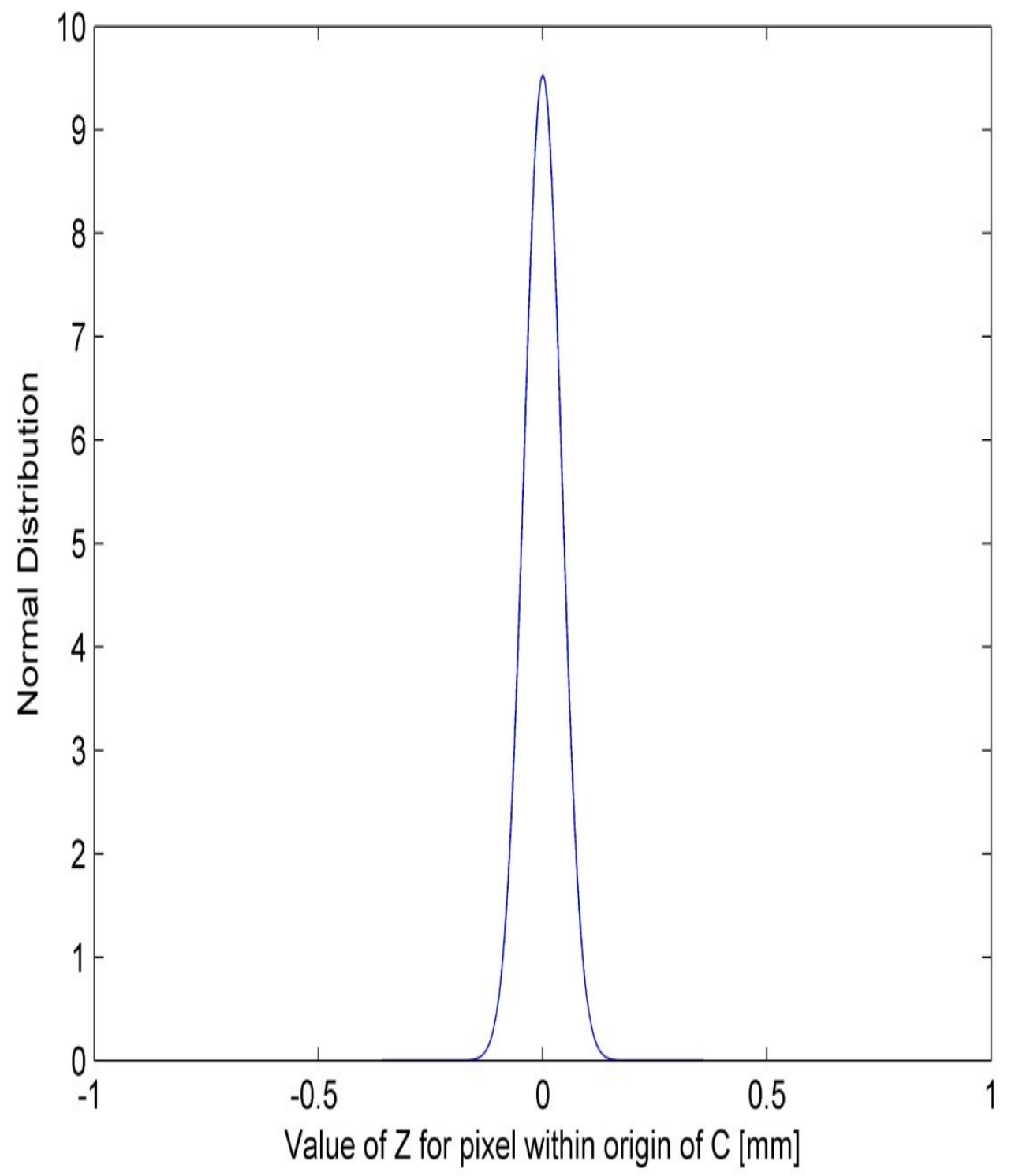

Figure 4-16: Normal distribution for values in $\mathrm{Z}$ axis at the center location of volume reconstruction from various images in different directions. Variance is $0.0122 \mathrm{~mm}^{2}$. Mean is $0.1718 \mathrm{~mm}$

During the spatial calibration process, Prager et al. [57] explain that the final goal is to obtain the transformation matrix of $T_{R \leftarrow P}$. It is still necessary to obtain $T_{C \leftarrow T}$ as well, even though the transformation matrix of $T_{C \leftarrow T}$ at the end is disregarded. A convenient arbitrary value can then be assigned for reconstruction. Thus, $\phi$ is the 12 elements vectors consisting of 6 parameters including positions $(\mathrm{X}, \mathrm{Y}, \mathrm{Z})$ and orientations $(\alpha, \beta, \gamma)$ inside the transformation matrix $T_{R \leftarrow P}$ and 6 parameters within the transformation matrix of $T_{C \leftarrow T}$. 
Because the coordinate system $\mathrm{C}$ can be placed in any orientation, the conditions of Equation 4.7 are still satisfied. Three orientation angles from transformation matrix of $T_{C \leftarrow T}$ are set to zero in Equation 4.9 from Equation 4.7 since they cannot be identified. Therefore, the reconstruction volume coordination system (C) would have the same orientation as ones in the transmitter or source coordinate system (T) as Figure 4-12.

In this chapter, the spatial calibration time is reduced so first objective can be met. The next steps are the data preprocessing and data analysis, while data pre-processing serves as the verification step for results from both the temporal and spatial calibrations. Data analysis deals with the elevation distance estimation and visualization system in 3D.

As mentioned in Section 3.7, challenges arise while scanning the real tissues containing the region of the coherent scattering during the operation of the speckle decorrelation method in the elevation distance estimation. Regions are rarely observed to only contain fully developed speckles (FDS) within real tissues. To overcome this limitation, the Cambridge heuristic method introduced by Gee et al. [16] is implemented, which accounts for both coherent and non-coherent scattering effects. Therefore, the real tissue correlation curve is derived from calibration on images with FDS due to the variation between them.

Afsham et al. [40], [41] mention an assumption also known as an isotropic medium, which signifies that coherency in both axial and lateral directions are the same as the elevation. With above condition, the amount of out-of-plane variation is deduced by knowing the amount of in-plane variation with the theoretical FDS curve in advance. In practice, the calibrated correlation curve is adjusted for every single patch. Bahramabadi [27] explains that this is done by comparing the in-plane correlation curve with their associated FDS so the compensation in the out-of-plane curve is obtained from their difference for each patch. Thus, the in-plane correlation is measured in terms of the percentage of coherent scattering in the patches then adjusted according to this percentage.

Afsham et al. [40], [41] note that the coherent effect on the elevation displacement is multiplied by a factor of four with axial or lateral displacement since the resolution cell is four times larger in the elevation direction. 
Difficulties arise from implementing this method due to the fixed size in pixels within the ultrasound B-mode images. As a result, the smallest distance that can be used in this thesis is the size of one pixel as $0.075 \mathrm{~mm}$ in both the axial and lateral directions. In addition, the smallest elevation distance is reduced to $0.3 \mathrm{~mm}$, as determined by the multiplication of $0.075 \mathrm{~mm}$ and 4. Bahramabadi and Gee et al. [16], [27] mention that their smallest elevation distances are $0.4 \mathrm{~mm}$.

It is first necessary to maintain the curve shape by implementing the curve adjustment algorithm. Then, the difference including images, positions, and orientations between two successive frames within in-plane motion must be considered to obtain the more accurate motion estimation. The implementation detail is introduced in next chapter.

By overcoming this difficulty, the elevation distance between patches is obtained from the adjustment of the elevation FDS calibrated curve by calculating the correlation between patches. In addition, the improved method in this thesis for reducing the standard deviation during the elevation distance estimation is provided in Chapter 5 and involves analyzing the data from the meat phantoms. For the $3 \mathrm{D}$ visualization, a lowresolution method is introduced as well. Instead of waiting for a long time for reconstructing the high-resolution voxel grid, a lower resolution one can be computed and displayed first. Once it becomes satisfied, the voxel grid with the higher resolution can be obtained later. 


\section{Chapter 5: Data Analysis and Results}

The first part of this section details two datasets from the speckle phantom to the meat phantoms between frames, along with the improved method for reducing the standard deviation of the elevation distance estimation.

The second part is related to the visualization of the meat phantoms in 3D. All have been implemented in Microsoft Visual Studio $2013 \mathrm{C}++$. The details of extra libraries for $\mathrm{C}++$ and system requirement specification are covered in Appendix A.

\subsection{Post Translation Data Verification}

Before proceeding to the next stage, the work and results from the last chapter requires verification.

Figure 5-1 illustrates a damp with a depth of $-11.04 \mathrm{~cm}$ for the beef phantom after the post-translation, which represent the highest position in the temporal calibration for matching the ultrasound image system and the PATRIOT sensor. Figure 5.2 relates to the post-translation for the chicken phantom with a maximum depth of $-13.61 \mathrm{~cm}$.

\subsection{Frames Selection from Data}

To estimate the out-of-plane motion with the decorrelation curve, Gee et al. [16] state that it is necessary to divide the B-scan images into patches due to inconsistencies in the width of the ultrasound resolution cell within the entire image. Such inconsistencies lead to variations in depth and it becomes necessary to have the calibration on all patches 
across the entire image. There are various approaches to dividing the images: Rivas et al. [92] introduce the detection of FDS region by moving the patches around images.

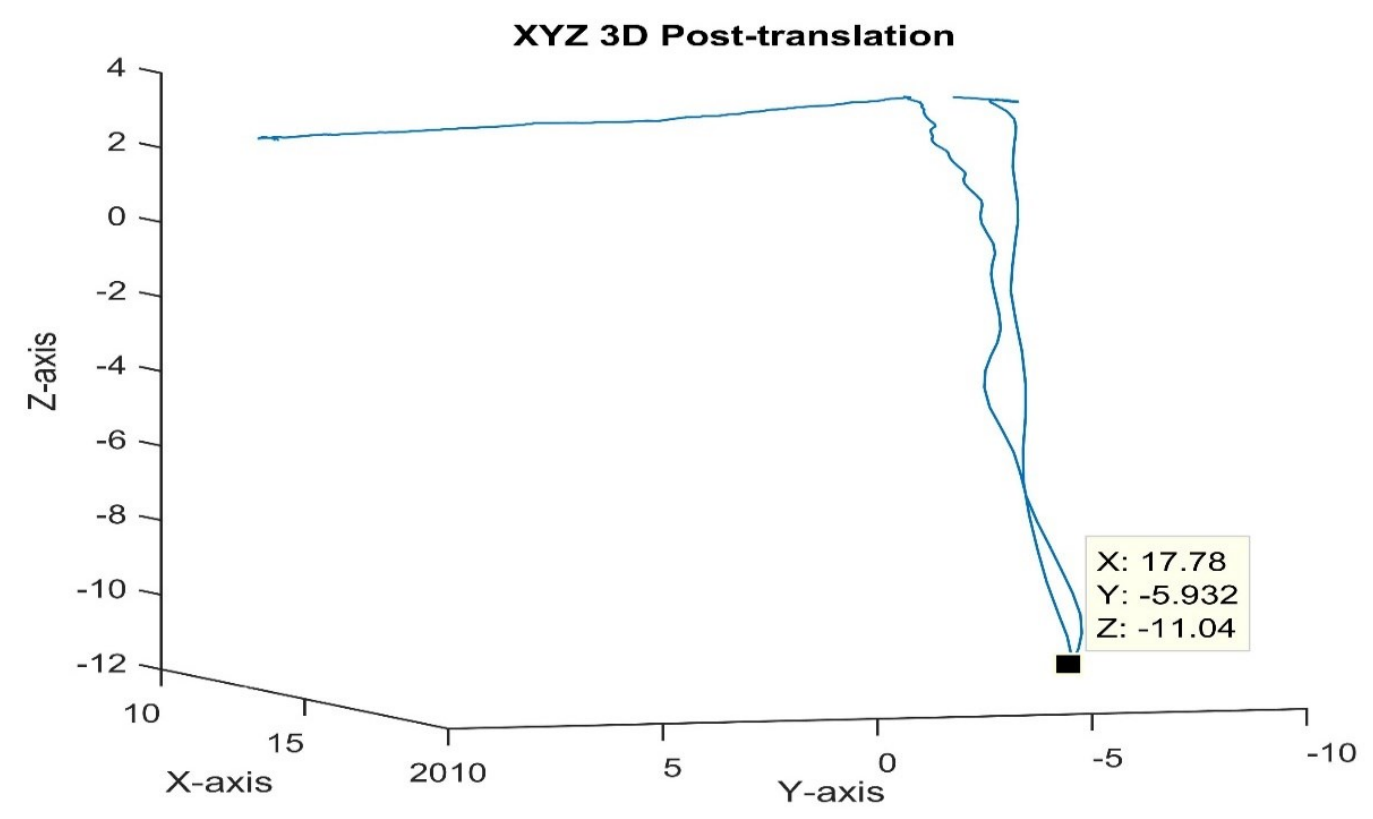

Figure 5-1: 3D coordinates for Beef phantom in post-translation [cm]

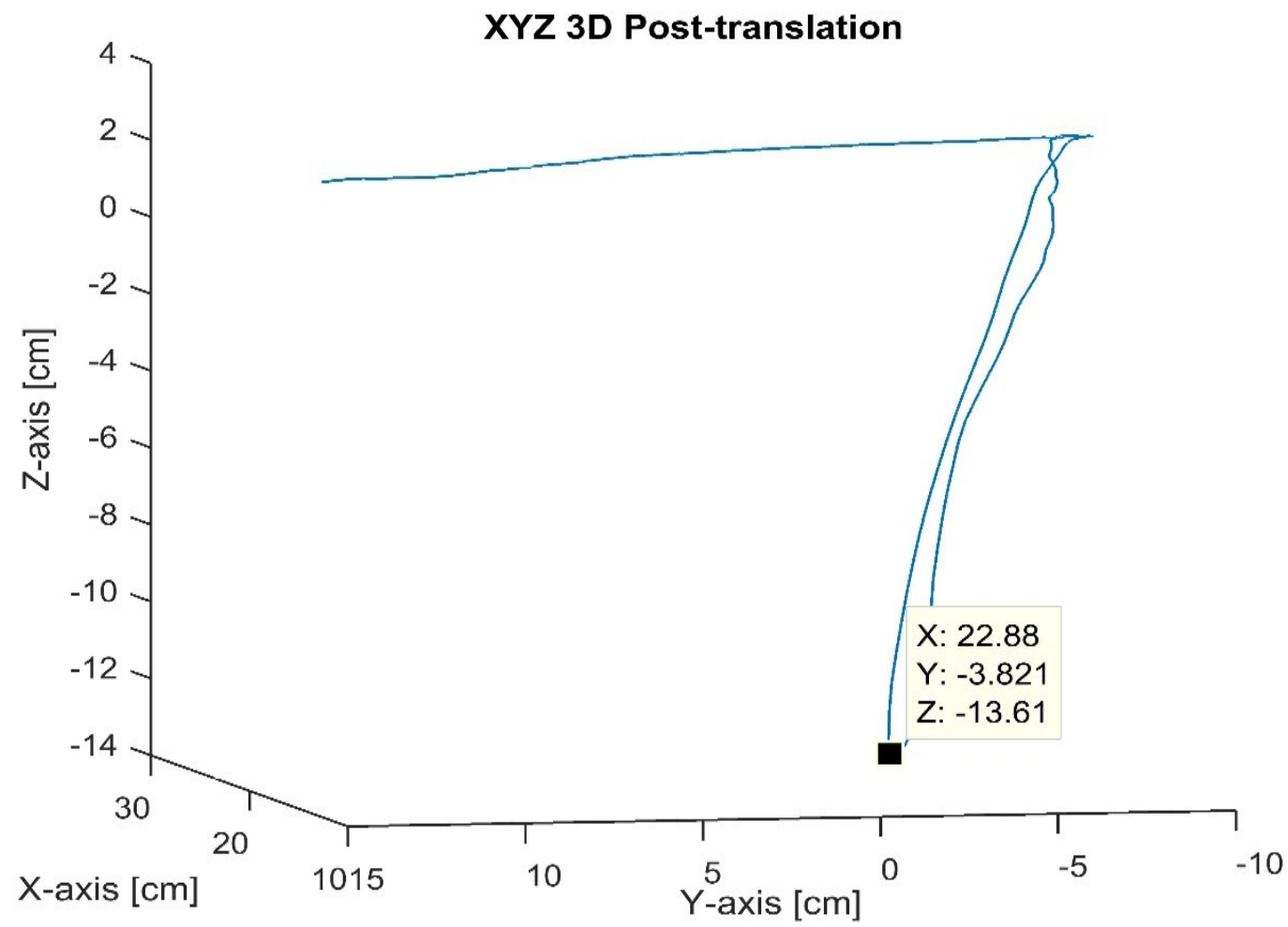

Figure 5-2: 3D coordinates for Chicken phantom in post-translation [cm] 


\begin{tabular}{|l|c|c|c|c|c|c|c|c|c|c|c|}
\hline 1 & 9 & 17 & 25 & 33 & 41 & 49 & 57 & 65 & 73 & 81 & 89 \\
\hline 2 & 10 & 18 & 26 & 34 & 42 & 50 & 58 & 66 & 74 & 82 & 90 \\
\hline 3 & 11 & 19 & 26 & 35 & 43 & 51 & 59 & 67 & 75 & 82 & 91 \\
\hline 4 & 12 & 20 & 28 & 36 & 44 & 52 & 60 & 68 & 76 & 84 & 92 \\
\hline 5 & 13 & 21 & 29 & 37 & 45 & 53 & 61 & 69 & 77 & 85 & 93 \\
\hline 6 & 14 & 22 & 30 & 38 & 46 & 54 & 62 & 70 & 78 & 86 & 94 \\
\hline 7 & 15 & 24 & 32 & 40 & 48 & 56 & 64 & 72 & 80 & 88 & 96 \\
\hline
\end{tabular}

Figure 5-3: Dividing an image into 96 patches for B-scan in speckle phantom

All images were divided into patches as twelve columns and eight rows; the fully developed speckle (FDS) and non-fully developed speckle (FDS) were both considered, meaning there were ninety-six patches in total. The order began from the patch 1, 9, 17 and 25 in the first row to 2, 1018 and 26 in the second row then continued. There was a general overview in Figure 5-3. The selection of the patches was dependent on the ROI for the test phantoms including the speckle phantom and meat phantoms. The locations of corresponding patches between speckle and meat phantoms were aligned. Additionally, the size of patches was different in both beef and chicken phantoms. The following were steps for selecting the frames from data including intensities and locations of patches for our analysis.

1) The center locations of the patches in each image were calculated for eight rows and twelve columns with 96 patches in total.

2) The position readings from the EM sensor were implemented with Equation 4.6 and 4.8 then combined with the result from the spatial calibration in the spatial phantom to obtain the center of the patches in the $3 \mathrm{D}$ locations. 
3) Bahramabadi and Gee et al. [16], [27] describe how the distances between the patches across all frames in each image are calculated. The distances included the elevation, axial, and lateral directions. They were associated with the $\mathrm{z}, \mathrm{y}$ and $\mathrm{x}$-axes in the image plane coordinate $(\mathrm{P})$ as in Figure 4-12.

4) The frames were organized into eight categories according to the range of the elevation distance, which could be $0.075,0.15,0.225,0.3,0.375,0.45,0.525$ or $0.6 \mathrm{~mm}$. The ceiling was $0.6 \mathrm{~mm}$ so the change of the maximum elevation distance never exceeded this value.

5) Only the 100 frames were chosen randomly within the selected category if there were more frames within the specified elevation distance. If there were less than one hundred frames, it was required to increase the range of the elevation distance. Therefore, more frames were considered. In addition, the selected frames were out of the range of temporal collaboration to ensure the validity of data.

This was the first step for reducing the marginal errors during the elevation distance estimation in the method brought forth in this thesis. The purpose of doing this was to represent a point in the decorrelation curve with these 100 samples, which were the building blocks in all of data sets in the thesis for the elevation distance estimation through the speckle decoration method.

\subsection{Calibration on FDS Decorrelation Curve}

The decorrelation curves in the speckle phantom from the selected frames needed to first be generated. The correlations between each patch were calculated with the known elevation distances. Approximations are based on the image intensity of the B-scan $\mathrm{I}_{1}$ and $\mathrm{I}_{2}$ with respect to the elevation distance $\delta$ in the Gaussian model function as shown in Equations 2.7 and 2.9. Gee et al. [16] explain that having radio frequencies (RF) exist alongside B-scan images from the ultrasound system is an ideal situation, so the image intensity in gray scale was linear. In practice, Bahramabadi and Gao et al. [15], [27] note that this limitation occurred when the RF signal is unavailable on the most standard US system within the clinical application. 
Under such a condition, the radio frequency intensity data is compressed in a logarithmic scale. The range of pixel intensity values in the grayscale B-mode images is from 0 to 255. In general, Smith et al. [99] explain that this is required to convert the image intensity back to the linear scale from the logarithmic scale.

$$
I(i, j)=10^{\frac{P(i, j)}{L}}
$$

As shown in Equation 5.1, I ( $\mathrm{i}, \mathrm{j})$ represents the uncompressed intensity in the ith row and jth column of the image within the grayscale. $P(i, j)$ represents the compressed intensity in the B-scan image. $\mathrm{L}$ is the linearization factor converting the logarithmic scale to the grayscale from 0 to 255 within 0 to $65 \mathrm{~dB}$ range. The $65 \mathrm{~dB}$ is selected since the recorded radio frequency (RF) signal of Ultrasonix Tablet has the dynamic range of $65 \mathrm{~dB}$. A set of B-scan images was used with the known distances for the formation of the decorrelation curve across the elevation distances by implementing the Pearson correlation coefficient as depicted in Equation 5.2.

$$
\rho(d)=\frac{\sum_{i=1}^{m} \sum_{j=1}^{n}\left(I_{0}(i, j)-\overline{I_{0}}\right)\left(\mathrm{Id}_{\mathrm{d}}(\mathrm{i}, \mathrm{j})-\overline{I_{d}}\right)}{\sqrt{\sum_{i=1}^{m} \sum_{j=1}^{n}\left(I_{0}(i, j)-\overline{I_{0}}\right)^{2} \sum_{i=1}^{m} \sum_{j=1}^{n}\left(\mathrm{Id}_{\mathrm{d}}(\mathrm{i}, \mathrm{j})-\overline{I_{d}}\right)^{2}}}
$$

In Equation 5.2, $\rho$ (d) represents the normalized correlation. $\mathrm{I}_{\mathrm{o}}$ means the pixel intensity of a particular patch within a particular B-mode scan at the location o. $\mathrm{I}_{\mathrm{d}}$ indicates that the pixel intensity of a particular patch within a particular B-mode scan at location d. $\overline{I_{o}}$ and $\overline{I_{d}}$ are the mean intensities at the locations o and $\mathrm{d}$ respectively. The symbol i represents the index of the height and $j$ represents the index of the width of a particular patch.

\subsubsection{Improved Decorrelation Curve}

For each patch and its corresponding elevation distance, the correlation coefficients were summed up and averaged with respect to their elevation distances across several patches. 
In the elevation decorrelation curve, Bahramabadi and Conrath et al. [27], [100] explain that the correlation coefficient is obtained between the shifted patch and the original patch in the different frames for the in-plane motion. The maximum correlation is found and regarded as the in-plane transition between two frames.

In both the axial and lateral decorrelation curves in this thesis, the correlation coefficients were obtained between the shifted patches and the original patches in the same frame as the out-of-plane motion. The amount of movement in the axial or lateral direction was restrained with one pixel under the B-mode scan. The dimension of the pixel was a $0.075 \mathrm{~mm} \times 0.075 \mathrm{~mm}$ square, making the shortest achievable distances in both the axial and lateral directions $0.075 \mathrm{~mm}$. The given offsets for lateral and axial correlation coefficients were $0,0.075,0.15$, and $0.225 \mathrm{~mm}$.

The maximum correlation coefficients with their relative distances were selected for the axial and lateral decorrelation curves in the speckle phantom. This approach decreased variation in the standard deviation of a given correlation coefficient. Consequently, the slope became steeper so the values for the elevation distance estimation became closer. This was the second step for reducing the marginal errors during the elevation distance estimation in the method posited in this thesis.

The decorrelation curve for the speckle phantom in the axial direction is shown in Figure 5-4, and plotted with Matlab Curvefit Toolbox ${ }^{\circledR}$. The shape of the curve is more similar across the rows than the columns due to the locations of the patches. Gee et al. [16] observe that if the patch is positioned in the deeper location, there is more attenuation. The lateral decorrelation curve for the speckle phantom has been obtained in Figure 5-5 through the same method. As shown in Figures 5-4 and 5-5, the decorrelation curves in both the axial and lateral directions are more similar across the rows than columns, even though this is not readily observed.

The elevation decorrelation curve for the speckle phantom has been obtained through the same method. In Figure 5-6, the shapes of patches 5 and 13 are more similar to each other than patches 5 and 6 due to the location of patches from attenuation effects. In other words, the patches in the horizontal direction are more similar with respect each other than the ones in the vertical direction. 

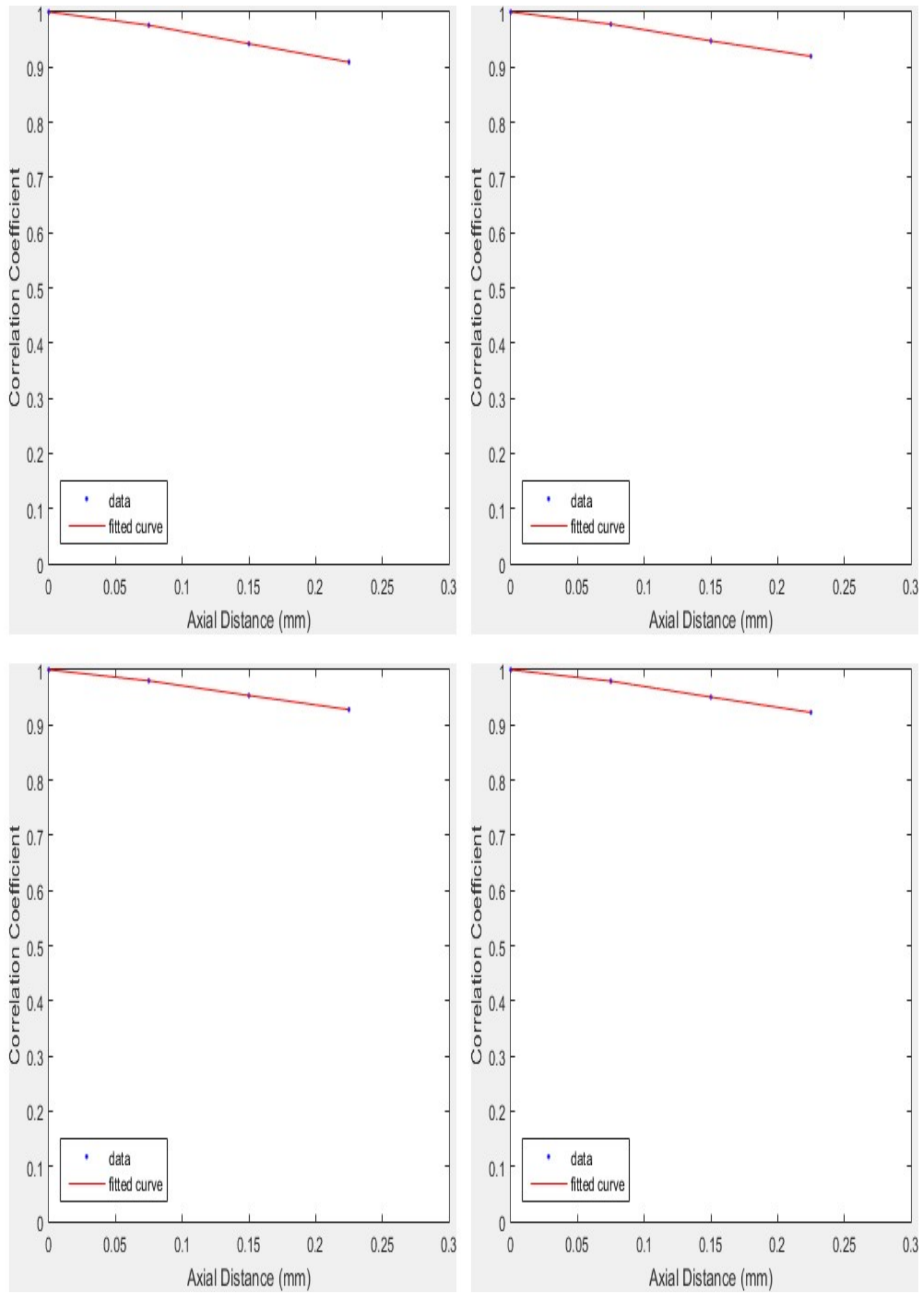

Figure 5-4: Axial decorrelation curve of speckle phantom for patch 5 and patch 13 in the first row.

Patch 6 and patch 14 are in the second row. 

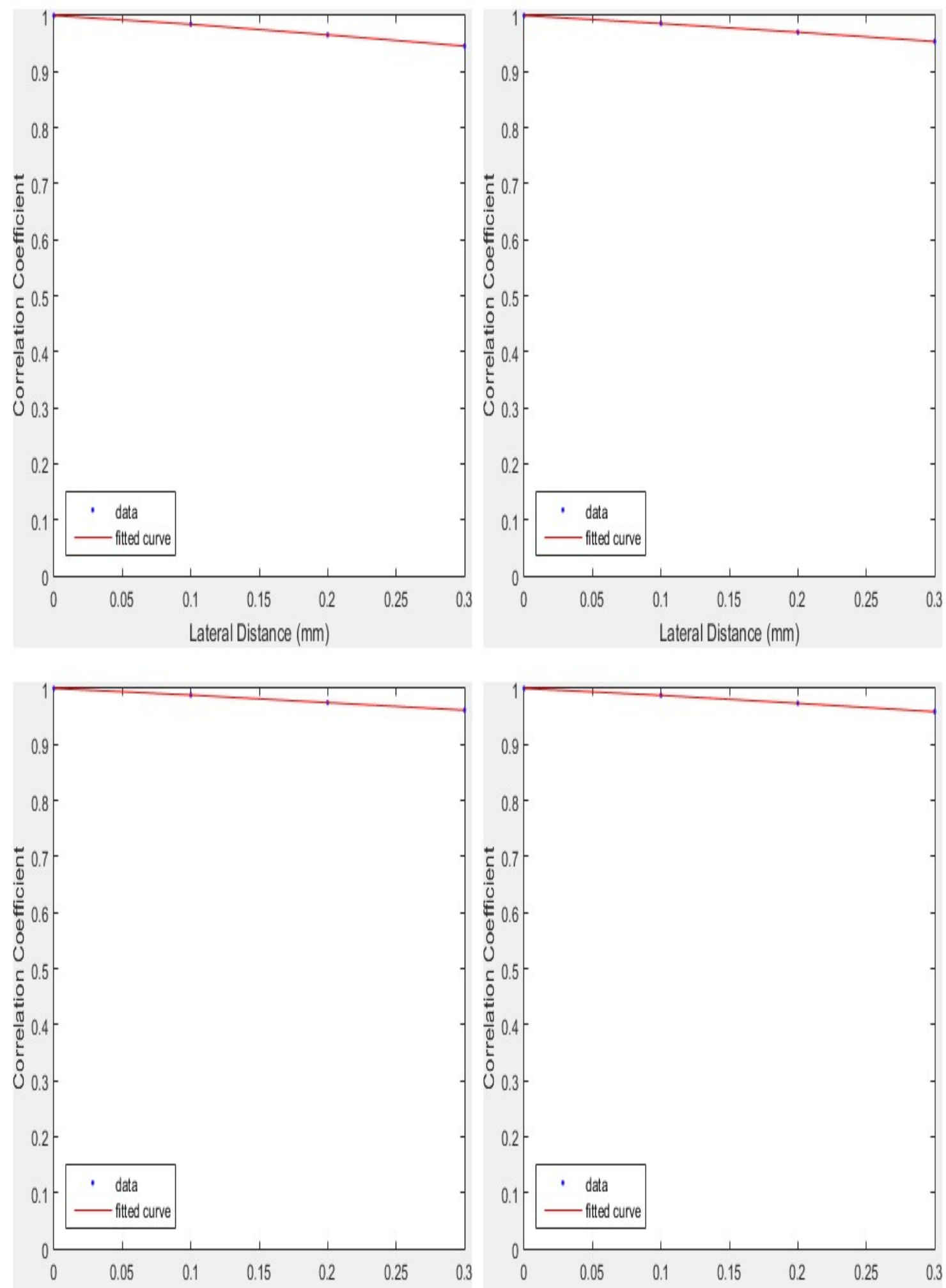

Figure 5-5: Lateral decorrelation curve of speckle phantom for patch 5 and patch 13 in the first row. Patch 6 and patch 14 are in the second row. 

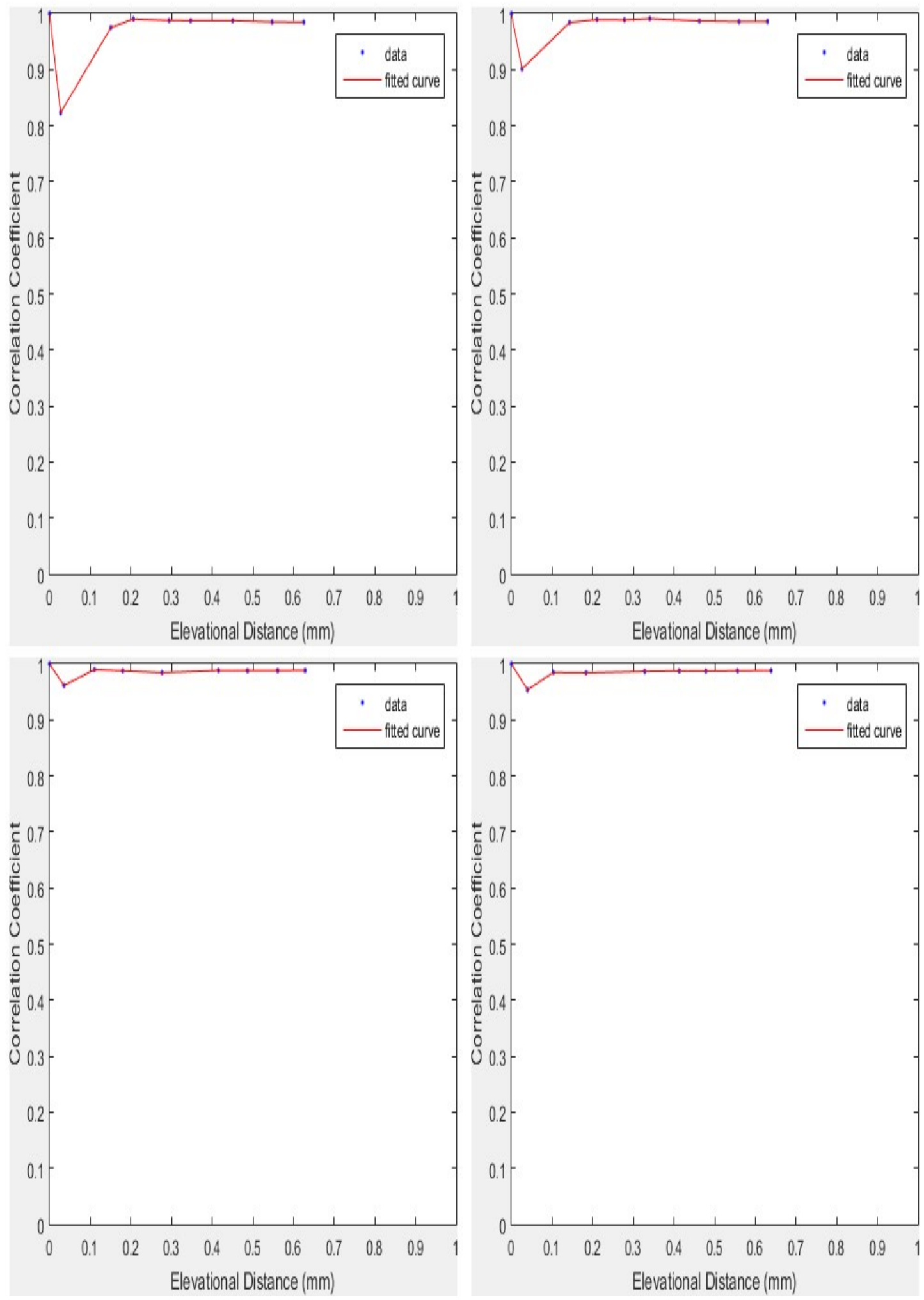

Figure 5-6: Elevation decorrelation curve of speckle phantom for patch 5 and patch 13 in the first row. Patch 6 and patch 14 are in the second row. 
Figures 5-4, 5-5, and 5-6, illustrate the resolution cells' widths are not consistent across all the B-scan images. In addition, there are more variations in the resolution cells vertically than that horizontally in the B-scan images. In support, Bahramabadi [27] notes that the ultrasound beam focus degrades as depth increases due to the attenuation effect.

\subsection{FDS Decorrelation Curve}

Bahramabadi and Gee et al. [16], [27] explain that the purpose of the decoration curve was to estimate the elevation distance for both chicken and beef phantoms between frames of ultrasound images. Elevation distance estimation errors occur when applying only the FDS decorrelation curve in real tissues because the existence of FDS is rare within real-tissue ultrasound images, and real tissues also contain coherent scattering. If only the Rayleigh scattering or FDS is considered, a lot of information is neglected in the data.

Gee et al. [16] overcome these obstacles by considering both the effects from the Rayleigh scattering and coherent scattering. This technique is also known as the Cambridge heuristic method and based on the ultrasound B-mode images instead of RF signals from Section 5.3.

There are image data sets in patch A and B in frame $i$ and frame $i+1$ for the elevation distance estimation in the real tissues. Gee et al. [16] mention that if there is only the FDS region within a patch, it can be represented by the equation as $\rho_{r}=\rho(A, B)$. The symbol of $\rho_{r}$ represents the Pearson correlation coefficient under the Rayleigh scattering condition. While dealing with the real tissue, the coherent scattering ought to increase with each patch.

Patches are modeled with the new equation as $A+\kappa B$ and $A-\kappa B$, where $\kappa$ is much greater than 0 and much smaller than 1 . It is expressed as $0<<\kappa<<1$. Thus, the new correlation coefficient is $\rho=\rho(A+\kappa B, A-\kappa B)$. The value of parameter $\kappa$ represents the amount of correlation from the coherent scattering between two patches over a certain distance. From Equation 5.3, the correlation coefficient represents the relationship between $\rho$ and $\rho_{r}$.

Using Equations 5.3 and 5.4, lateral and axial decoration curves were calculated at first for a specific patch A. Using an axial correlation $\rho_{a}$ with the respective axial shift $d_{a}$ 
and the expected correlation $\rho_{\mathrm{r}}$ from the FDS axial decorrelation curve, $\kappa_{a}$ could be calculated from Equation 5.4. The symbol $\kappa_{a}$ represents the coherent scattering in the patch $\mathrm{A}$ in the axial direction quantitatively. The value of $\kappa_{l}$ is achieved through the same procedure. Gee et al. [16] indicate that the assumption is based on the physical property of the isotropy. There should be the same amount of coherent scattering in the elevation distance $\mathrm{d}_{\mathrm{e}}$ as both the axial and lateral distances.

In this thesis, the elevation resolution was four times worse than the axial direction and lateral resolutions as the elevation distance was four times greater than both axial and lateral distances. They were represented as $4 \mathrm{~d}_{\mathrm{a}}$ and $4 \mathrm{~d}_{1}$.

$$
\begin{aligned}
& \rho=\frac{\left(1+\kappa^{2}\right) \rho+2 \kappa}{\left(1+\kappa^{2}\right)+2 \kappa \rho} \\
& \kappa=\frac{(1-\rho \rho)}{(\rho-\rho \cdot)}-\frac{\sqrt{\left(1-\rho^{2}\right)(1-\rho)^{2}}}{(\rho-\rho)} \\
& k_{e}(4 d)=0.5\left(k_{a}(d)+k_{1}(d)\right)
\end{aligned}
$$

The smallest possible displacement in both the axial and lateral directions in this thesis was $0.075 \mathrm{~mm}$, which was equivalent to the height and width of one pixel. A set of the displacements $(0 \mathrm{~mm}, 0.075 \mathrm{~mm}$, and $0.15 \mathrm{~mm})$ was used to calculate the amount of scattering in both the axial and lateral directions as $\kappa_{a}$ and $\kappa_{l}$. Therefore, the set of the matching displacements $(0 \mathrm{~mm}, 0.3 \mathrm{~mm}$ and $0.6 \mathrm{~mm})$ was found for the scattering in the elevation direction as $\kappa_{e}$. The average value has been taken for obtaining the final set value of $\kappa_{e}$ from $\kappa_{a}$ and $\kappa_{l}$ as illustrated in Equation 5.5. The adapted elevation decorrelation curve was formed for patch A by taking both the Rayleigh and coherent scatterings into account via Equation 5.3. Since it was in the B-scan images, the quantity of the displacement was restrained to the size of one pixel. Consequently, there were three correlation points as $0 \mathrm{~mm}, 0.3 \mathrm{~mm}$ and $0.6 \mathrm{~mm}$ for forming the adapted elevation decorrelation curve.

According to Gee et al. [16] the adapted elevation decorrelation curve was created by taking six data points in the range of $0 \mathrm{~mm}$ to $0.3 \mathrm{~mm}$ and 0.3 to $0.6 \mathrm{~mm}$ in this thesis. 
They were used as the reference points to observe the changes in quantity of maintaining the original shape of the curve. If the difference between the FDS correlation and adapted correlation in the elevation distance was $0.3 \mathrm{~mm}$ or represented by $\mathrm{D}_{0.3}$, the amount of variation for the points with respect to the set of elevation distances was $0.075 \mathrm{~mm}, 0.15$ $\mathrm{mm}$ and $0.225 \mathrm{~mm}$, and was denoted as $0.25 \mathrm{D}_{0.3}, 0.5 \mathrm{D}_{0.3}$, and $0.75 \mathrm{D}_{0.3}$. The same technique could be applied to the data points on the $0.6 \mathrm{~mm}$ in the elevation distance as well. The amount of shift of the points with respect to the set of elevation distance was $0.375 \mathrm{~mm}, 0.45 \mathrm{~mm}$ and $0.525 \mathrm{~mm}$, and was denoted by $0.25 \mathrm{D}_{0.6}, 0.5 \mathrm{D}_{0.6}$, and $0.75 \mathrm{D}_{0.6}$.

There are currently no journal articles that discuss reducing the standard deviation in the Cambridge heuristic method. With the reduced standard deviation, Hajian-Tilaki et al. [17] infer that lower marginal errors can be obtained for better precision in clinical studies. In using the improved method, the slopes between two last points in Figure 5-7 and Figure 5-8 are -2.035 and -2.216 . When the slope becomes steeper, there is less variation in the standard deviation of the elevation distance.

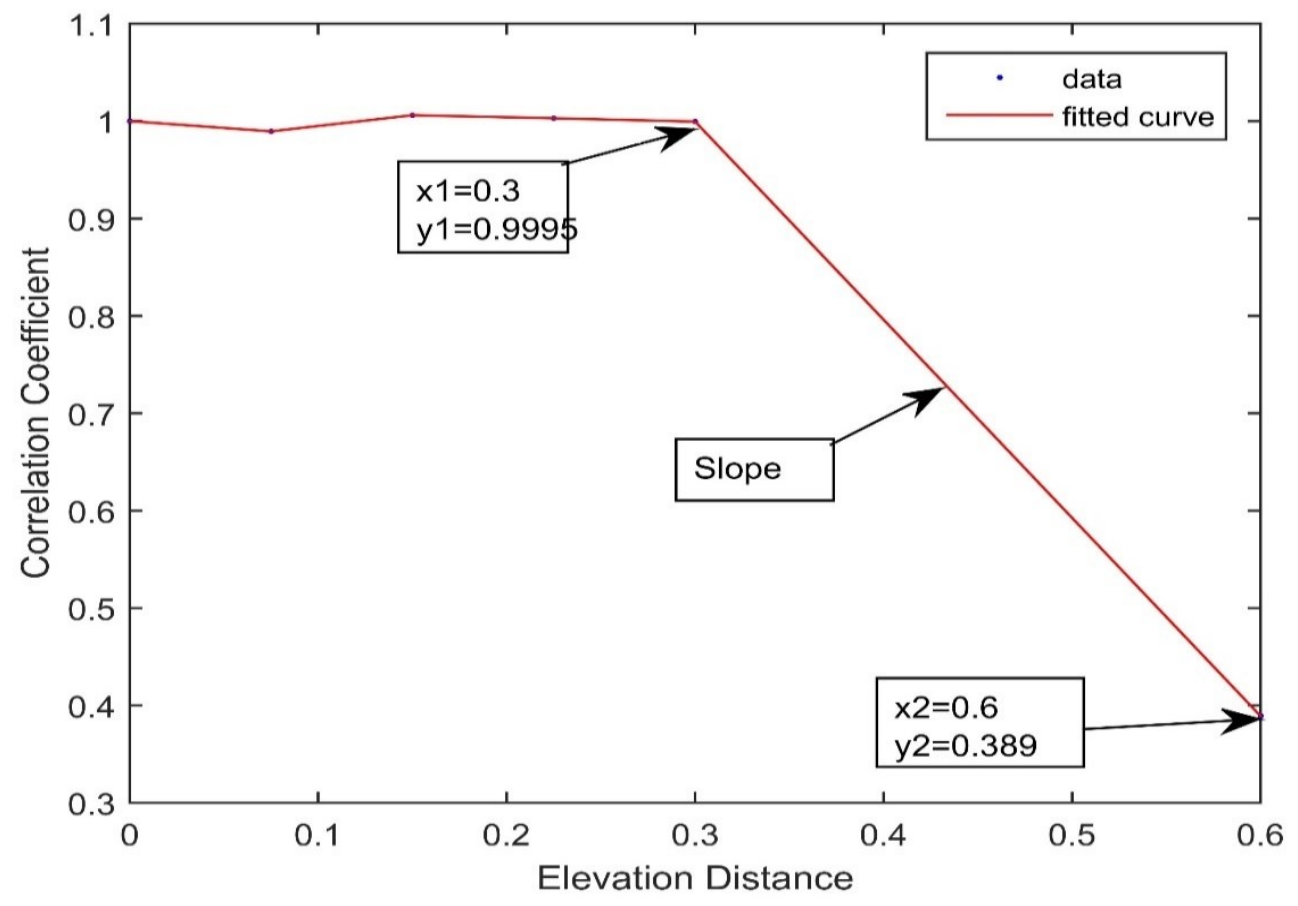

Figure 5-7: FDS adaptive decorrelation curve in the original elevation distance estimation 


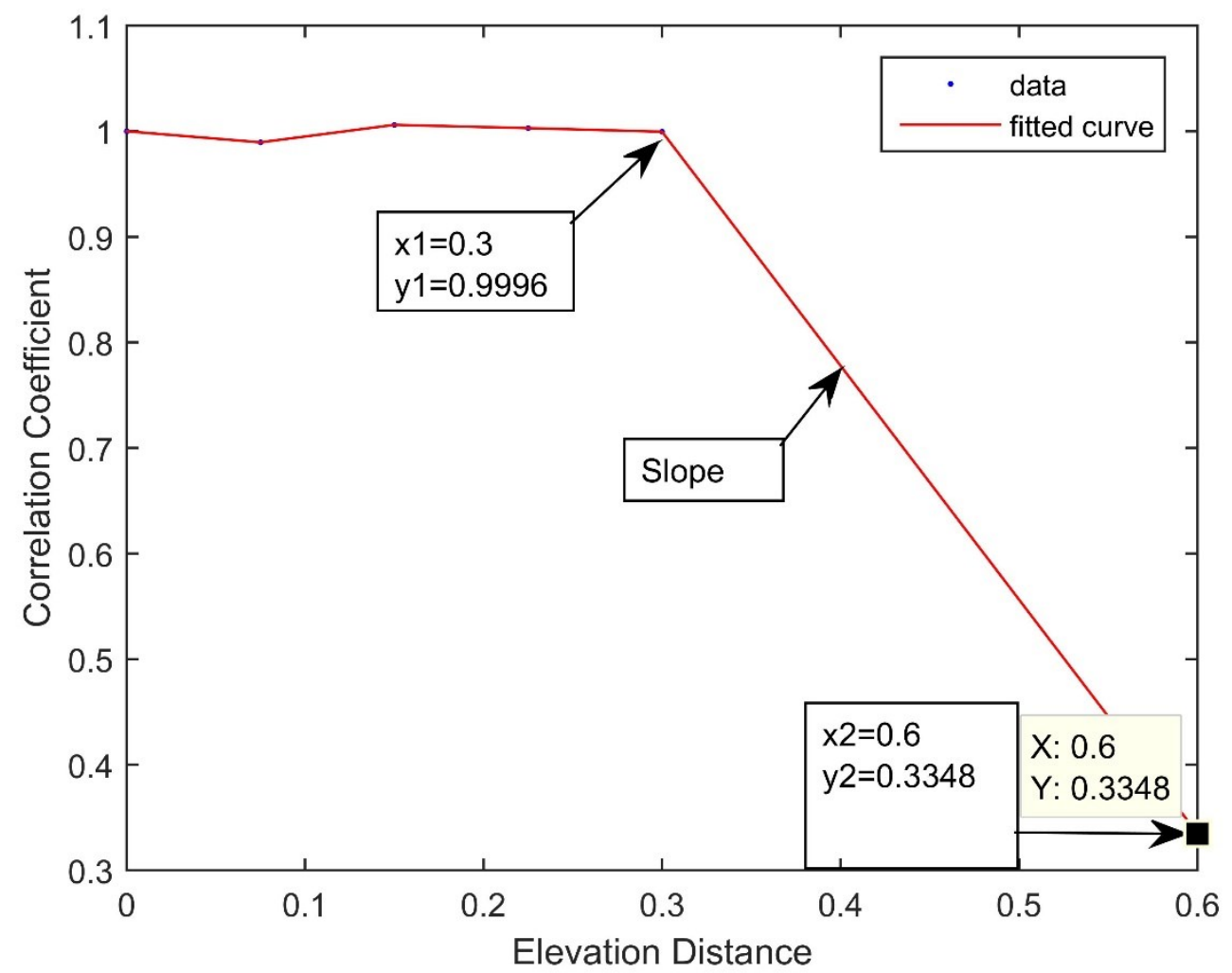

Figure 5-8: FDS adaptive decorrelation curve in the improved elevation distance estimation

\subsection{D Visualization Implementation}

One of the tasks for this thesis was to create the low-resolution 3D voxel grids by collecting the sequence of the 2D ultrasound B-mode images. As mentioned in Section 3.4 , there are different ways including voxel nearest neighbor (VNN), pixel nearest neighbor (PNN) and function based method (FBM) to present the positions and orientation of the anatomical structures in the 3D space. It is currently advantageous to employ the low-resolution method first to perform the verification at an earlier stage.

Our algorithm selected two nodes from its neighbor pixels to reduce complexity in the volume reconstruction, and each image was divided into ninety-six patches. The midpoints between different patches on the same frame were first calculated: As illustrated in Figure 5-9, the midpoints between the patch 1 represented by (o1x, oly, $\mathrm{o} 1 \mathrm{z})$ and patch 2 represented by (o2x, o2y, o2z) at frame $\mathrm{n}$ and their corresponding patches at frame $\mathrm{n}+1$ were calculated through Equations 5.6 and 5.7. The value of $t a, t b$ 
and $q$ were 0.5 . The new voxel position for a particular patch in 3D space was then calculated by averaging the midpoint obtained with Equation 5.8. After obtaining the voxel position and intensity in the grids for various frames, they were converted into a kv6 file format, originally created by Silverman [101], [102] for the Voxlap voxel rendering engine and Slab6 voxel editor. It was based on ray tracing as introduced in Section 3.5.3.

$$
\begin{aligned}
& \left(\begin{array}{l}
v 2 b x \\
v 2 b y \\
v 2 b z
\end{array}\right)=\left(\begin{array}{l}
o 1 x \\
o 1 y \\
o 1 z
\end{array}\right)+t b\left(\begin{array}{l}
o 2 x-o 1 x \\
o 2 y-o 1 y \\
o 2 z-o 1 z
\end{array}\right) \\
& \left(\begin{array}{l}
v 2 a x \\
v 2 a y \\
v 2 a z
\end{array}\right)=\left(\begin{array}{l}
p 1 x \\
p 1 y \\
p 1 z
\end{array}\right)+t a\left(\begin{array}{l}
p 2 x-p 1 x \\
p 2 y-p 1 y \\
p 2 z-p 1 z
\end{array}\right) \\
& \left(\begin{array}{l}
\mathrm{v} 1 \mathrm{x} \\
\mathrm{v} 1 \mathrm{y} \\
\mathrm{v} 1 \mathrm{z}
\end{array}\right)=\left(\begin{array}{l}
\mathrm{v} 2 \mathrm{ax} \\
\mathrm{v} 2 \mathrm{ay} \\
\mathrm{v} 2 \mathrm{az}
\end{array}\right)+\mathrm{q}\left(\begin{array}{l}
\mathrm{v} 2 \mathrm{bx}-\mathrm{v} 2 \mathrm{ax} \\
\mathrm{v} 2 \mathrm{by}-\mathrm{v} 2 \mathrm{ay} \\
\mathrm{v} 2 \mathrm{bz}-\mathrm{v} 2 \mathrm{az}
\end{array}\right)
\end{aligned}
$$

Either the open source rendering engine or in-house visualization system was modified or developed for displaying the 3D anatomical structures for the target of interest. The specification of the kv6 file format was listed in Appendix B. The low resolution of the 3D reconstruction of the target at a particular frame was displayed with the Slab6 voxel editor in Figure 5-10. The low resolution of 3D reconstruction of the target in series was listed with the Voxlap voxel rendering engine in Figure 5-11 with the temporal calibration. The third objective of this thesis was therefore met by displaying the lowresolution voxel grids so that verification could be completed in the initial stage. 


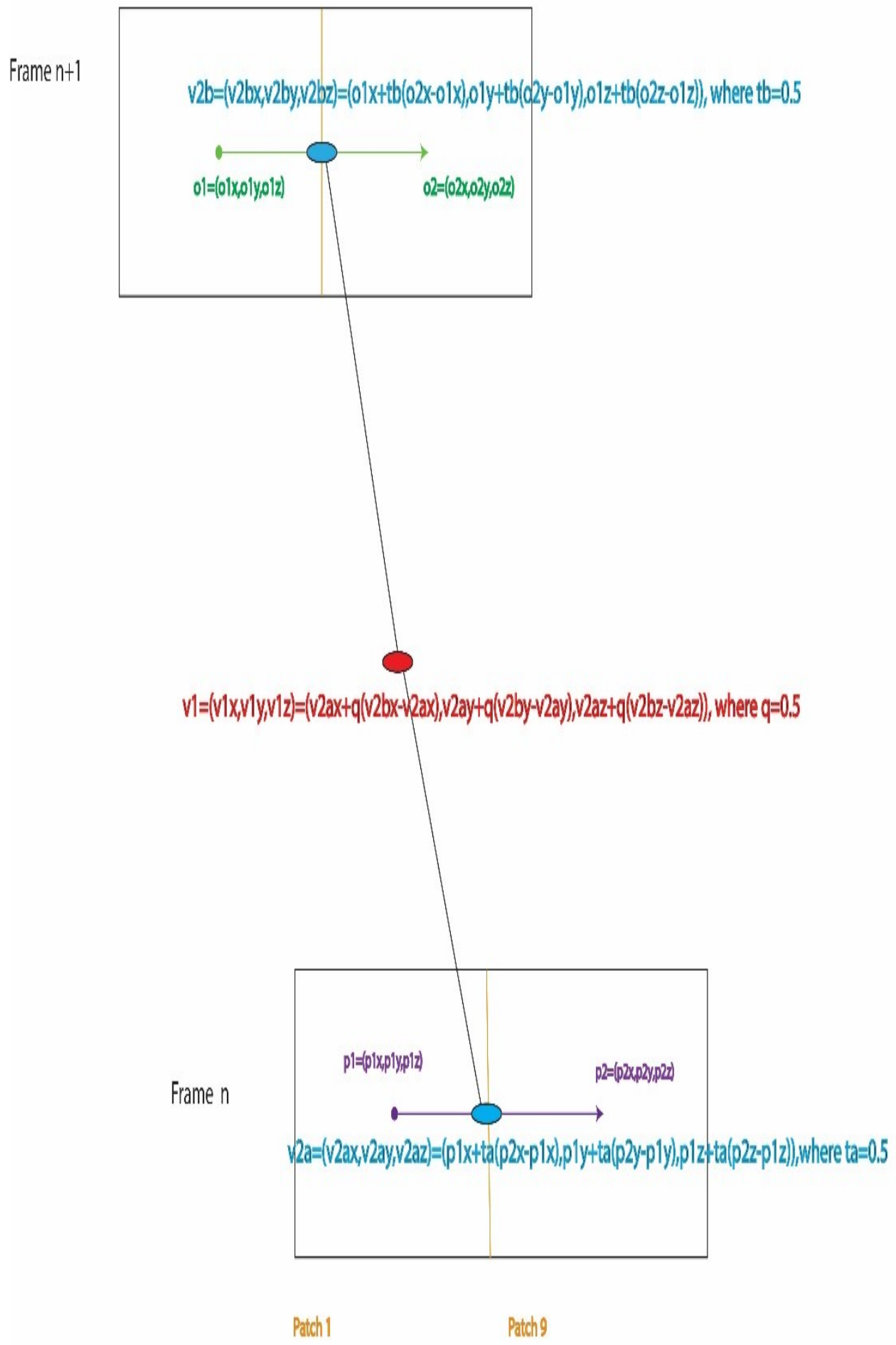

Figure 5-9: An improved algorithm for calculating voxel position and its intensity in volume reconstruction 


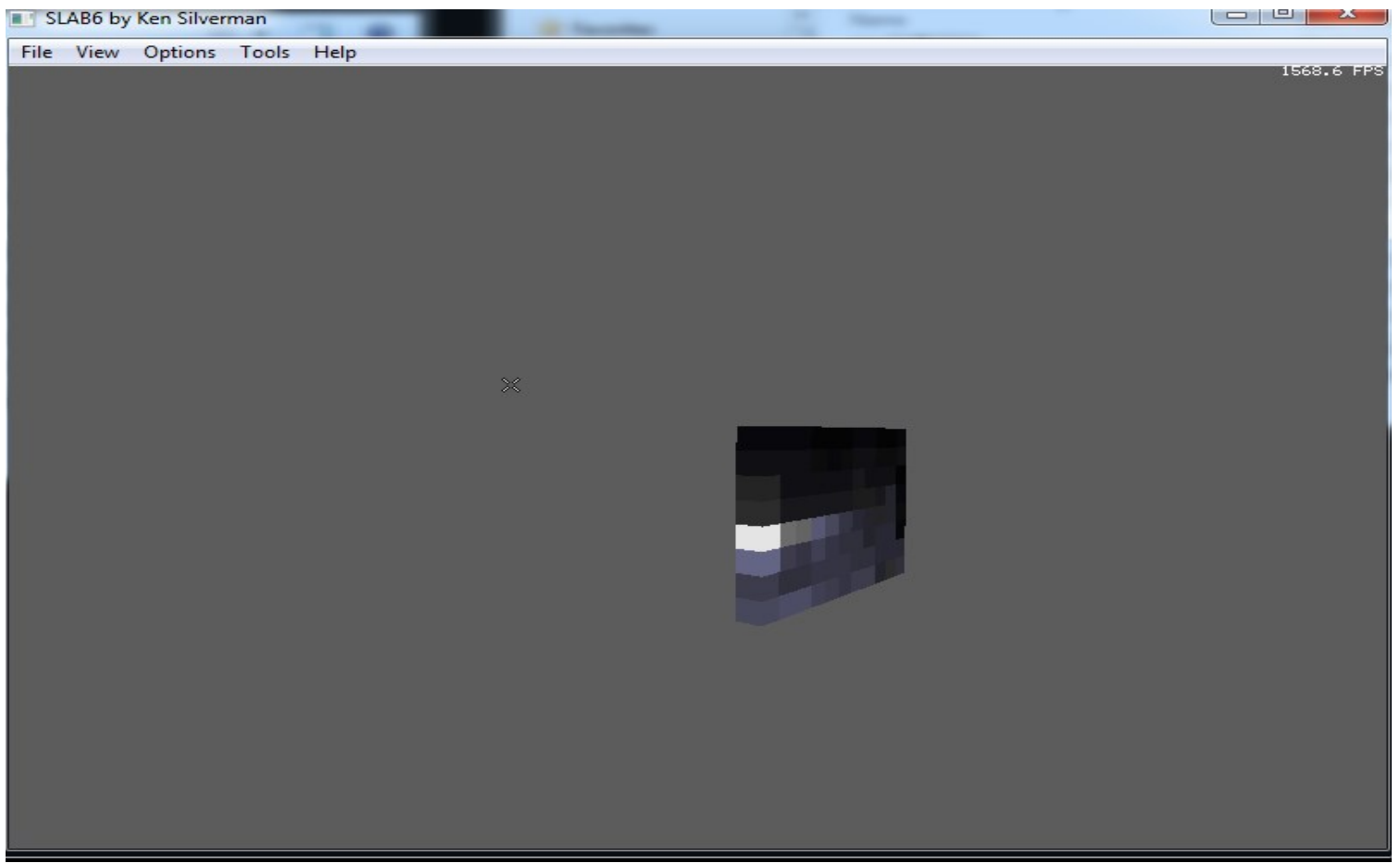

Figure 5-10: Low-resolution algorithm for displaying the 3D structure of the targeted anatomy at a particular frame

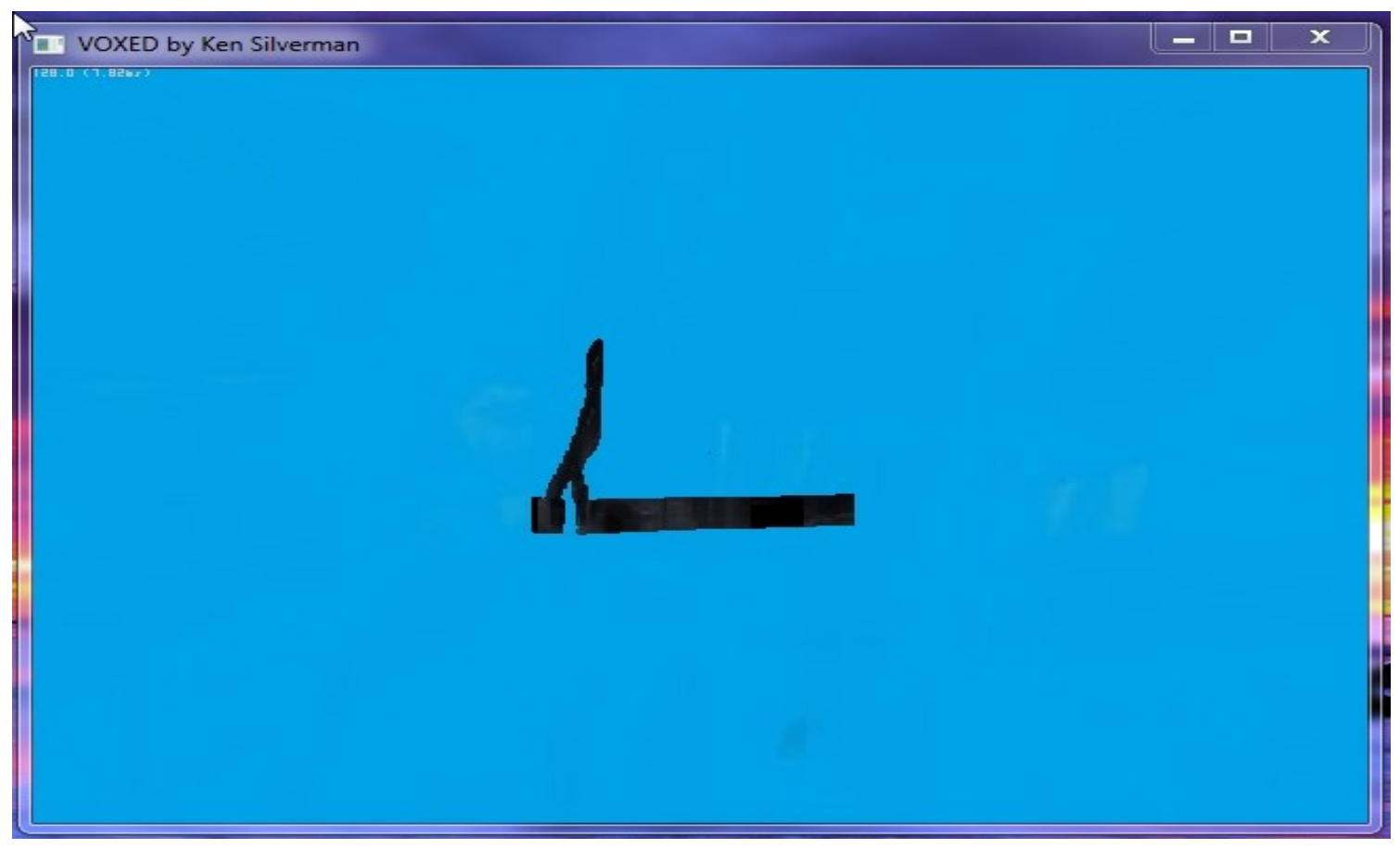

Figure 5-11: Low-resolution algorithm for displaying the 3D structure of the targeted anatomy in series 


\subsection{Data Analysis and Evaluation}

There were two analyses to be drawn from this information: First, the decorrelation curves were formed in axial, lateral and elevation directions as introduced in Section 5.2. The 100 sample frames were selected from the speckle, beef, and chicken phantoms for the elevation distance set of $0.075 \mathrm{~mm}, 0.15 \mathrm{~mm}, 0.225 \mathrm{~mm}, 0.3 \mathrm{~mm}, 0.375 \mathrm{~mm}, 0.45$ $\mathrm{mm}, 0.525 \mathrm{~mm}$ and $0.6 \mathrm{~mm}$. Second, the adapted correlation curve was formed for the specific patch as mentioned in Sections 5.3 and 5.4. By calculating the correlation coefficients between two corresponding patches, the elevation distance could be obtained from the FDS adaptive decorrelation curve. After obtaining each distance via data analysis, the results were averaged to produce a single distance for each pair of the scan.

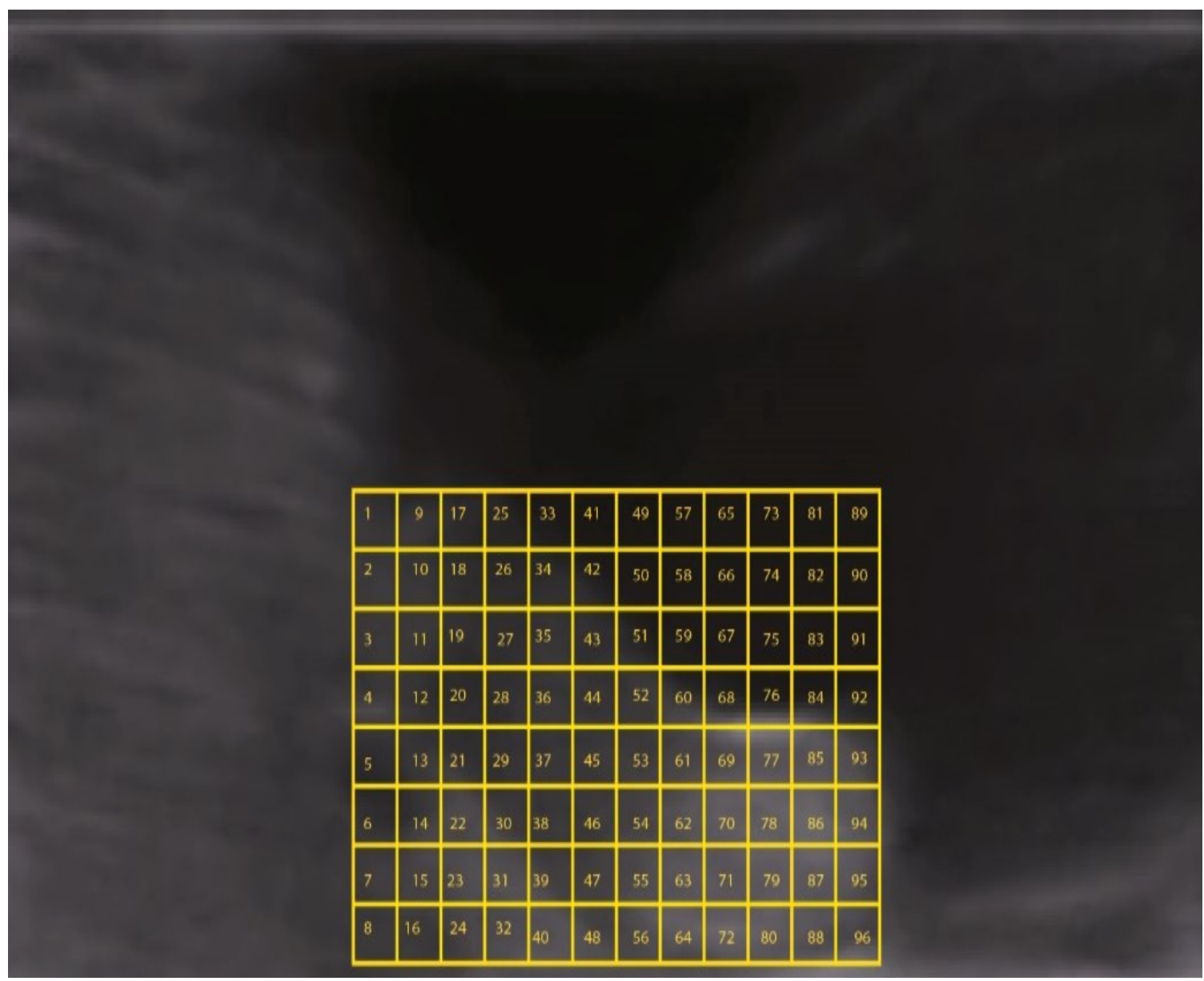

Figure 5-12: Relocation of ROI in Beef phantom at frame 317

The estimated elevation distance was obtained after the test phantom measurements were completed, and was compared to the measured elevation distance captured from the 
PATRIOT electromagnetic sensor as a baseline. In this thesis, the PATRIOT magnetic sensor data was considered as the gold standard, and no errors were considered to have arisen from this data. For the elevation distance estimation in meat phantoms, the center location and size of the patch were different for data analysis in both chicken and beef due to the ROI in B-scan images as displayed in Figure 5-12 and Figure 5-13. Consequently, their locations in both the speckle phantom and meat phantoms were different than in Figure 5-3.

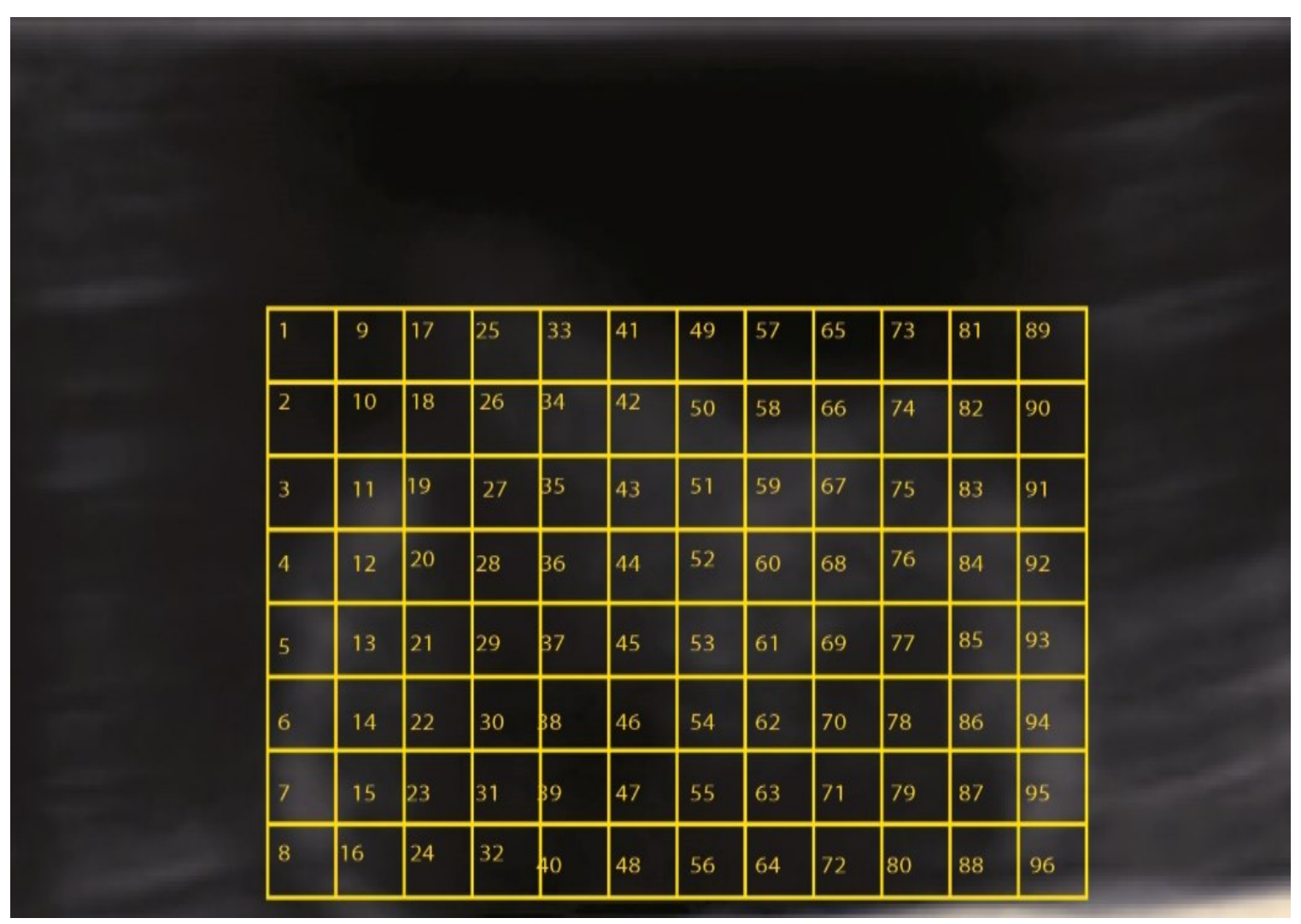

Figure 5-13: Relocation of ROI in Chicken phantom at frame 296

As discussed earlier, the estimated distance between the pair of frames was calculated by averaging all of the data from the FDS adaptive decorrelation curve in the elevation direction under the same patch after specifying the correlation coefficients. The theoretical and calculated distances with their associated standard deviation for the original elevation distance estimation were displayed in Table 5-1. 
Table 5-1: Original Elevation Distance Estimation

\begin{tabular}{|l|l|l|l|l|l|l|l|l|}
\hline $\begin{array}{l}\text { Frame } \\
\text { Separation } \\
\begin{array}{l}\text { Type of } \\
\text { phantom }\end{array}\end{array}$ & $0.075 \mathrm{~mm}$ & $0.15 \mathrm{~mm}$ & $0.225 \mathrm{~mm}$ & $0.3 \mathrm{~mm}$ & $0.375 \mathrm{~mm}$ & $0.45 \mathrm{~mm}$ & $0.525 \mathrm{~mm}$ & $0.6 \mathrm{~mm}$ \\
\hline $\begin{array}{l}\text { Chicken } \\
\text { [mm] }\end{array}$ & 0.2478 & 0.1502 & 0.194 & 0.2795 & 0.365 & 0.3214 & 0.3279 & 0.2842 \\
\hline $\begin{array}{l}\text { Chicken } \\
\text { SD }\end{array}$ & \pm 0.1101 & \pm 0.0860 & \pm 0.0626 & \pm 0.1516 & \pm 0.1092 & \pm 0.0948 & \pm 0.0869 & \pm 0.0606 \\
\hline Beef [mm $]$ & 0.2486 & 0.1492 & 0.2094 & 0.3297 & 0.3572 & 0.3836 & 0.4495 & 0.4834 \\
\hline Beef SD & \pm 0.1184 & \pm 0.0869 & \pm 0.11 & \pm 0.1434 & \pm 0.1439 & \pm 0.1773 & \pm 0.2044 & \pm 0.2277 \\
\hline
\end{tabular}

Table 5-2: Improved Elevation Distance Estimation

\begin{tabular}{|l|l|l|l|l|l|l|l|l|}
\hline $\begin{array}{l}\text { Frame } \\
\text { Separation } \\
\begin{array}{l}\text { Type of } \\
\text { phantom }\end{array}\end{array}$ & $0.075 \mathrm{~mm}$ & $0.15 \mathrm{~mm}$ & $0.225 \mathrm{~mm}$ & $0.3 \mathrm{~mm}$ & $0.375 \mathrm{~mm}$ & $0.45 \mathrm{~mm}$ & $0.525 \mathrm{~mm}$ & $0.6 \mathrm{~mm}$ \\
\hline $\begin{array}{l}\text { Chicken } \\
\text { [mm] }\end{array}$ & 0.2479 & 0.1515 & 0.1876 & 0.2783 & 0.3456 & 0.3621 & 0.3173 & 0.3446 \\
\hline $\begin{array}{l}\text { Chicken } \\
\text { SD }\end{array}$ & \pm 0.1102 & \pm 0.0803 & \pm 0.0556 & \pm 0.1469 & \pm 0.1051 & \pm 0.112 & \pm 0.0832 & \pm 0.077 \\
\hline Beef [mm] & 0.249 & 0.1467 & 0.2141 & 0.2795 & 0.2916 & 0.3275 & 0.3691 & 0.382 \\
\hline Beef SD & \pm 0.1185 & \pm 0.0823 & \pm 0.1046 & \pm 0.1177 & \pm 0.1158 & \pm 0.1464 & \pm 0.1631 & \pm 0.1744 \\
\hline
\end{tabular}

The improved elevation distance estimation is displayed in Table 5-2. Table 5-1 is associated with Figures 5-14 and 5-16 as the beef and chicken data sets for the original elevation distance estimation. Table 5-2 is associated with Figures 5-15 and 5-17 for the improved elevation distance estimation in the beef and chicken data sets. In Tables 5-1 and 5-2, the means of inter-frame estimations are shown in the center with the error bar $( \pm)$, representing the standard deviation. 


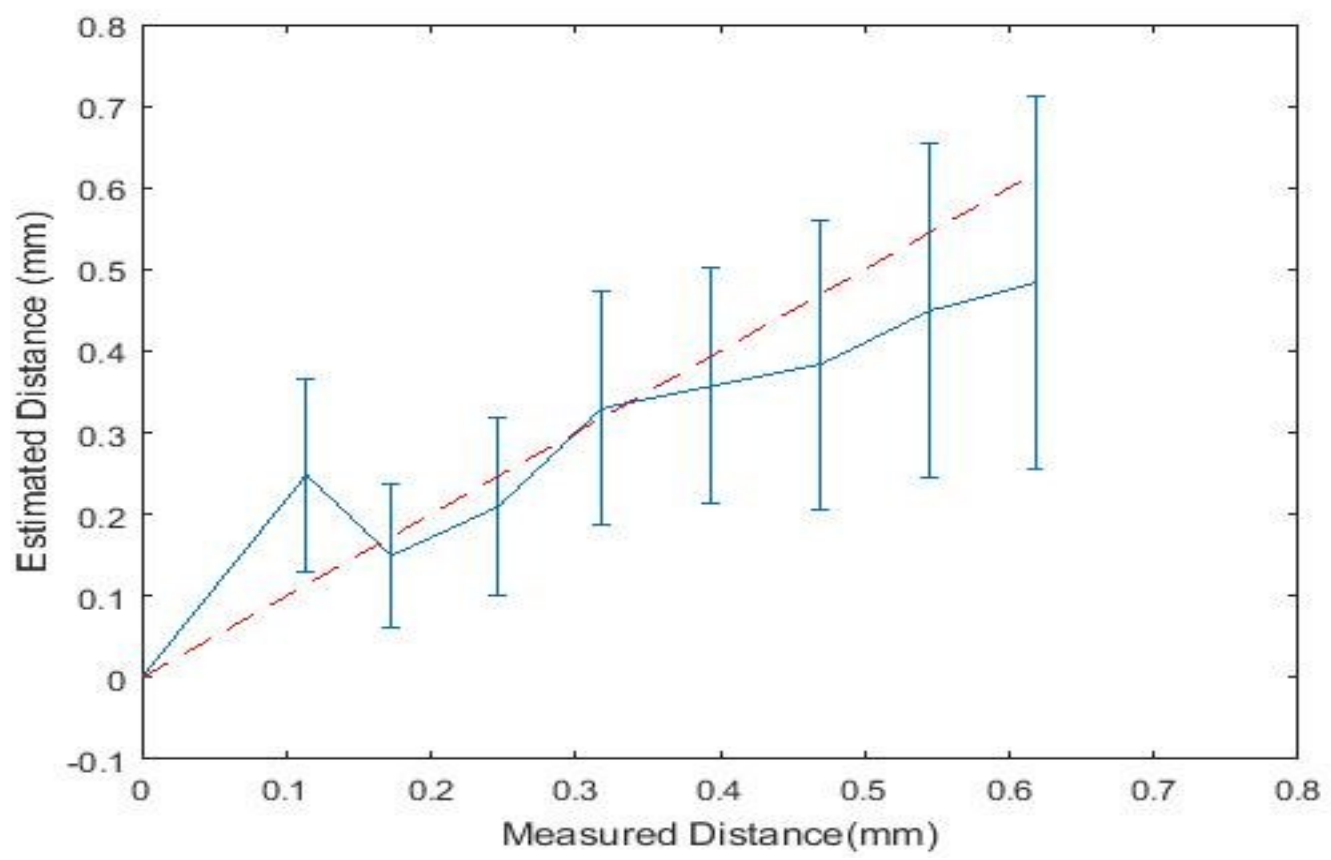

Figure 5-14: Original elevation distance estimation on Beef phantom

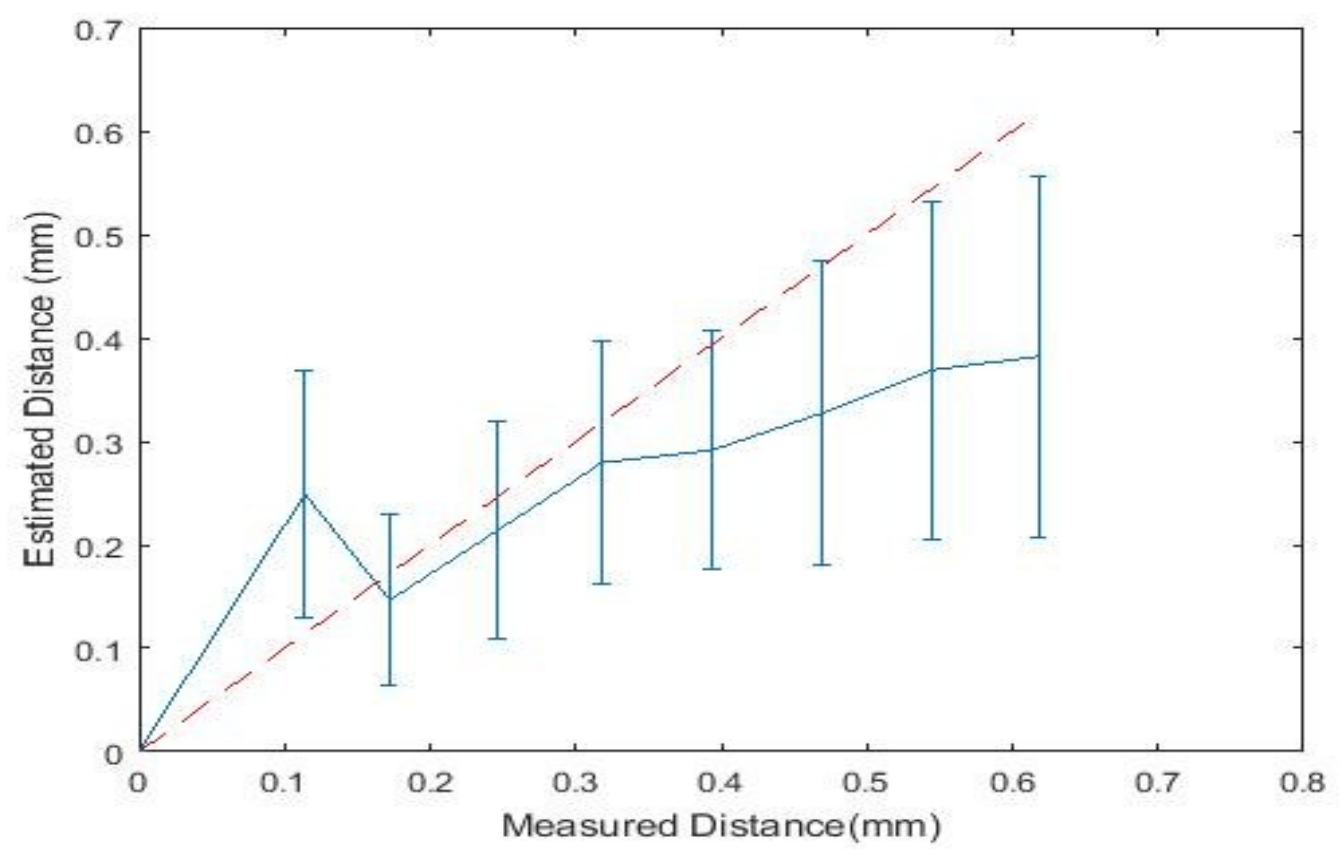

Figure 5-15: Improved elevation distance estimation on Beef phantom 


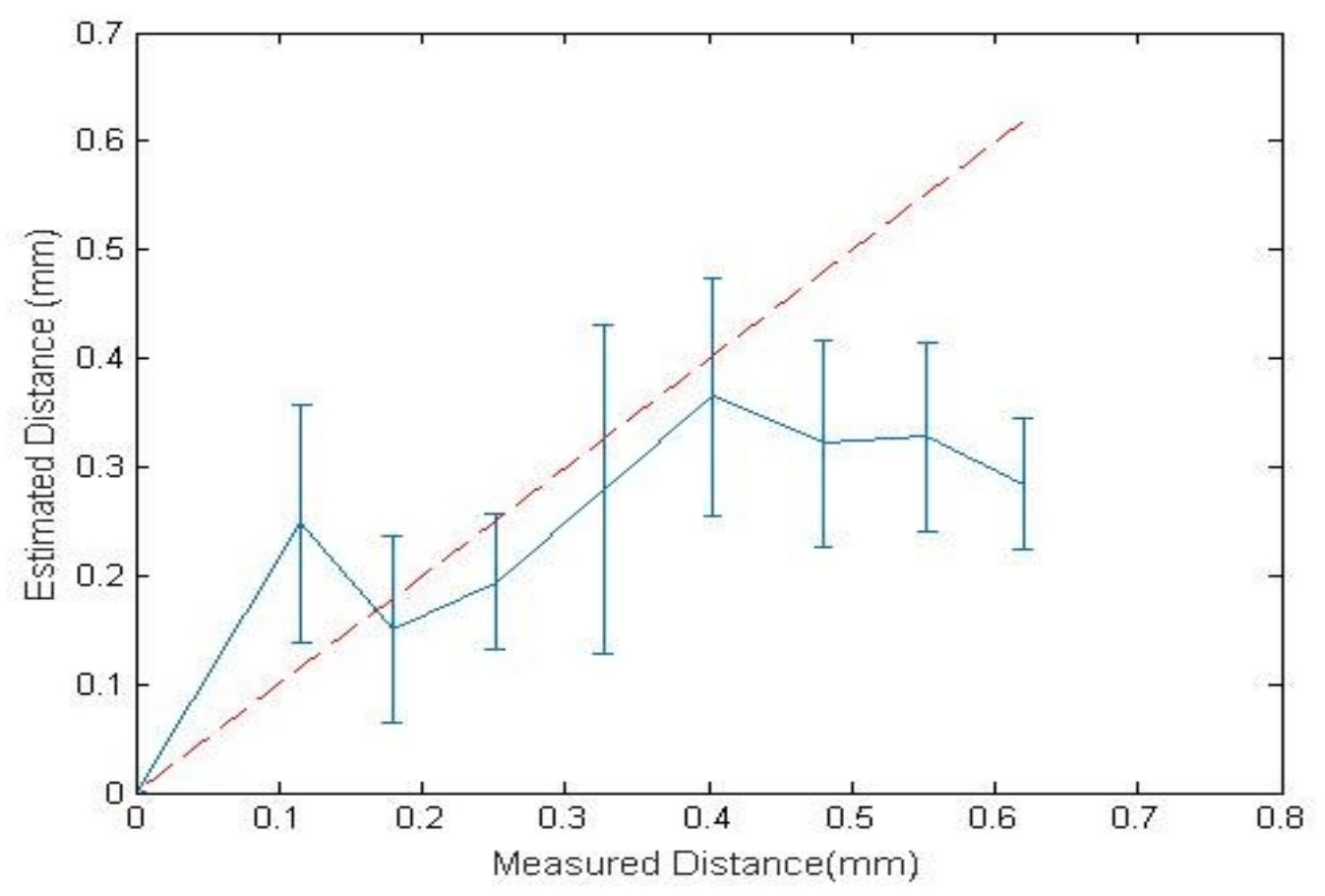

Figure 5-16: Original elevation distance estimation on chicken phantom

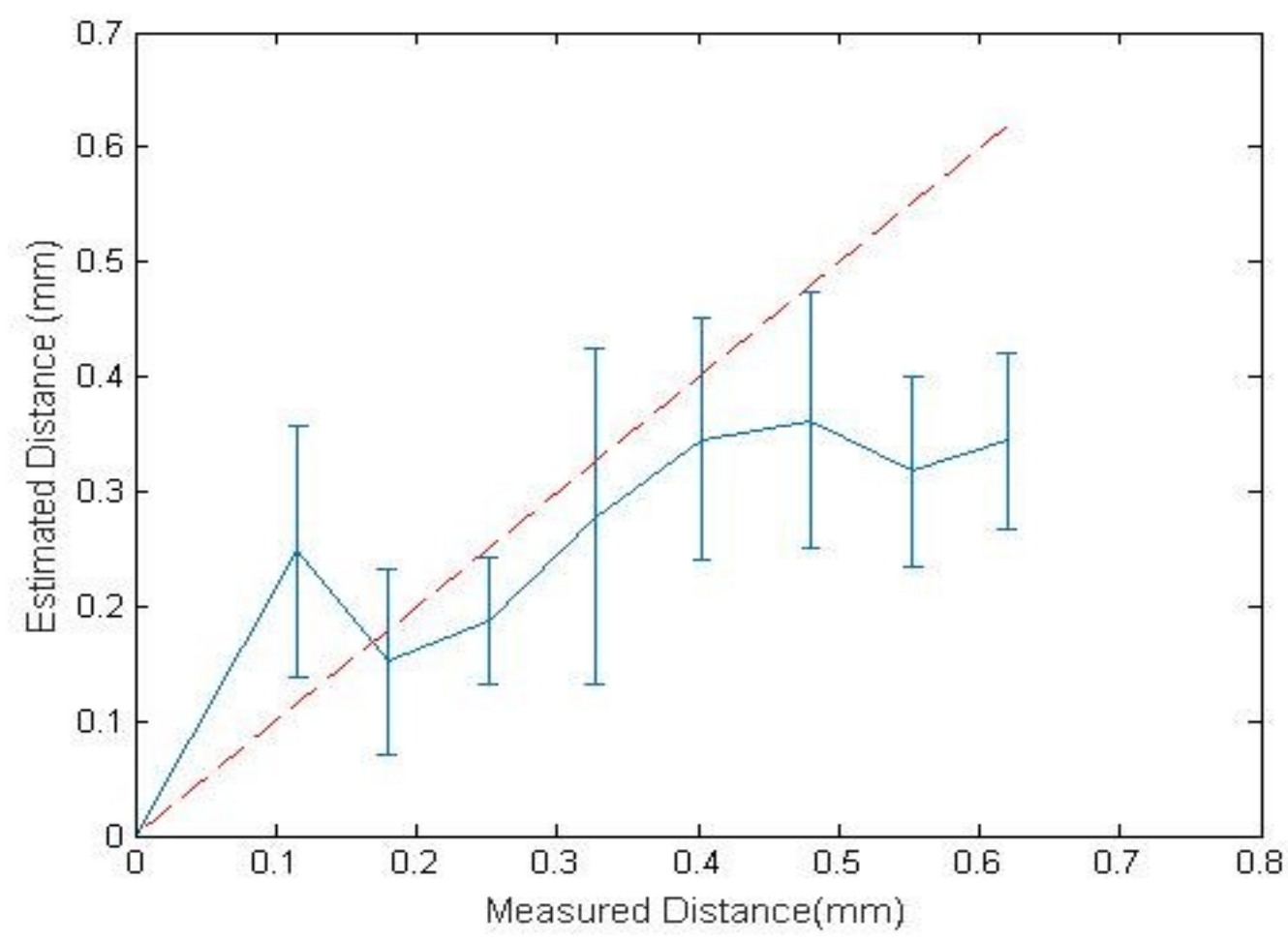

Figure 5-17: Improved elevation distance estimation on chicken phantom 
The estimation accuracy is related to the percentage error. In the chicken data set, the ranges of the percentage errors in both the original and improved methods are 0.13$13.78 \%$ and $1-16.62 \%$ respectively. In the beef data set, the ranges of the percentage errors in both the original and improved methods are $0.53-9.9 \%$ and $2.2-22.24 \%$ respectively. The standard deviation is related to the percentage change in each estimated distance within Table 5-3. In chicken, the percentage of change decreases in a range of $3.1 \%$ to $11.18 \%$. For beef, the percentage change decreases in the range of $4.91 \%$ to $19.53 \%$. Consequently, our method reduces the standard deviation if the range of the distance estimation is between $0.15 \mathrm{~mm}$ to $0.375 \mathrm{~mm}$. Therefore, the second objective of reducing the standard deviation is met.

Table 5-3: Percentage change in chicken and beef phantoms for standard deviation

\begin{tabular}{|l|l|l|l|l|l|l|l|l|}
\hline $\begin{array}{l}\text { Frame } \\
\text { Separation\Ty } \\
\text { pe of phantom }\end{array}$ & $\begin{array}{l}0.075 \\
\mathrm{~mm}\end{array}$ & $\begin{array}{l}0.15 \\
\mathrm{~mm}\end{array}$ & $0.225 \mathrm{~mm}$ & $0.3 \mathrm{~mm}$ & $0.375 \mathrm{~mm}$ & $0.45 \mathrm{~mm}$ & 0.525 & $0.6 \mathrm{~mm}$ \\
\hline Chicken [\%] & 0.091 & -6.628 & -11.1821 & -3.100 & -3.755 & 18.144 & -4.257 & 27.0627 \\
\hline Beef [\%] & 0.0845 & -5.293 & -4.909 & -17.922 & -19.527 & -17.428 & -20.206 & -23.408 \\
\hline
\end{tabular}

\subsection{Discussion}

For the spatial calibration, the procedure offered in this thesis reduced the spatial calibration time to 10 seconds in addition to a reduction in the overall calibration time. Reducing the spatial calibration time maintained consistency in the data acquisition process since the properties of phantoms including stiffness or elasticity degraded over time. Therefore, our results might vary after completing the data acquisition process due to these changes. In a more clinical and practical sense, the wait time for sonographers and patients was reduced.

Figures 5-14 to 5-17 illustrated that this method worked for the elevation offsets from $0.15 \mathrm{~mm}$ to $0.375 \mathrm{~mm}$ by reducing the standard deviation during the elevation distance estimation. As mentioned in Section 5.4, the steepness of slopes in the FDS adaptive decorrelation curve had an impact on the estimated elevation distance. When the slope became steeper at the given correlation numbers, the variation in the estimated distance 
was reduced too. As the variation in estimated distance was reduced, the standard deviation and its variance decreased as well.

There are three reasons errors occur during the elevation distance estimation of the freehand US scan. First, the average of the correlation measurement is taken in the decorrelation curve for the elevation distance estimation. The risk of the resultant measurement was incongruous from the mean between two patches.

Second, Gee et al. and Bahramabadi [16], [27] noted that systematic error could occur. From Figure 5-18, a pair of patches was separated by a small distance $\mathrm{d}_{1}$. If the measured correlation coefficient was higher than the expected correlation coefficient, the lookup became invalid due to no matched distance. If the measured correlation coefficient was lower than the expected correlation coefficient, there could be an overestimation of elevation distance $d_{1}{ }^{\prime}$. In the scenario where two patches were separated by a very large elevation distance $\mathrm{d}_{2}$, if the measured correlation coefficient was higher than expected correlation coefficient, there would be an underestimation of the elevation distance estimation $\mathrm{d}_{2}{ }^{\prime}$. If the measured correlation coefficient was lower than expected correlation coefficient, there would be an overestimation of the elevation distance estimation $\mathrm{d}_{2}{ }^{\prime \prime}$.

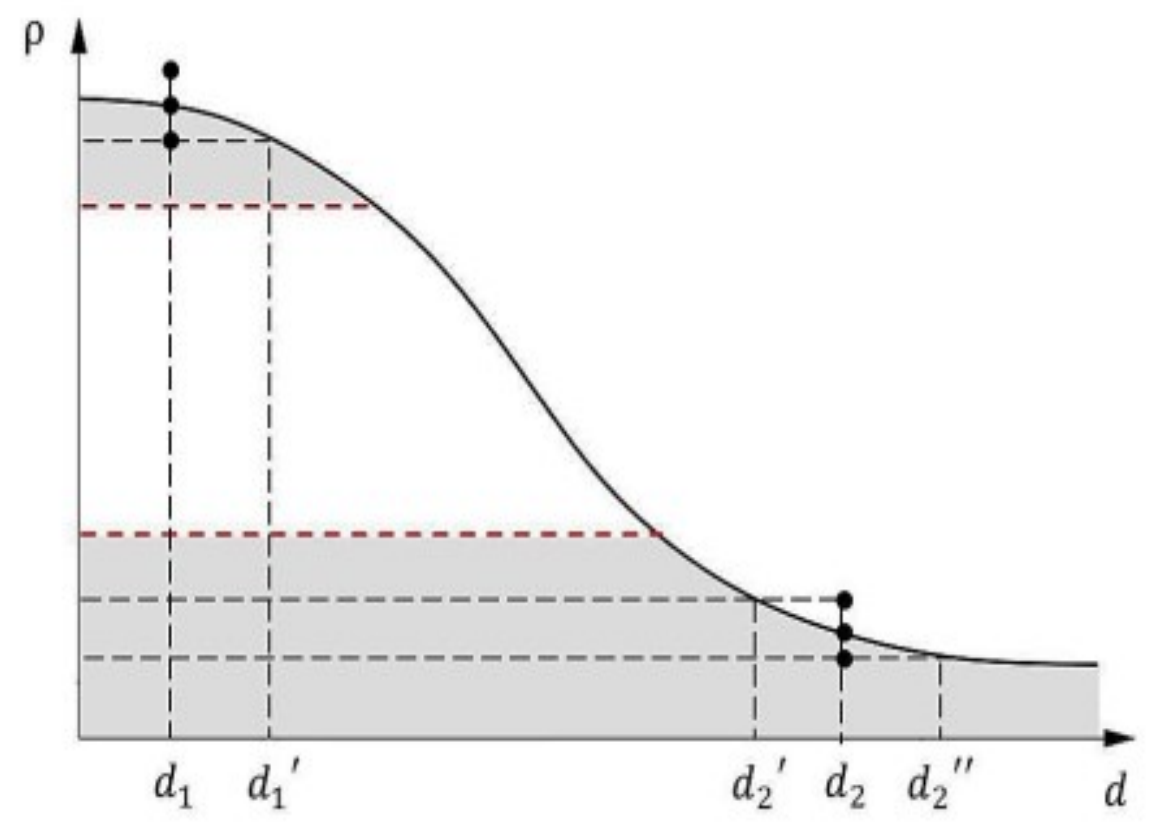

Figure 5-18: Systematic error for large or small distance in the elevation direction from [27] 
Finally, Gee et al. [16] described that the location of patches can affect the accuracy of the result at the end as well due to the attenuation of the ultrasound signals. The deeper the phantom was located, the more attenuated the signals would be, further adding noise to images.

There was a difference in equipment setup between this thesis and the Cambridge heuristic method due to the maximum depth of the image. The maximum depth chosen for this thesis was $3 \mathrm{~cm}$. Therefore, it caused the scale factors to change from 0.1 $\mathrm{mm} /$ pixel to $0.075 \mathrm{~mm} /$ pixel, denoted as $\mathrm{Sx}$ and Sy. The acquired data after post translation was thus smaller than what was observed in Cambridge heuristic method according to Equation 4.6. In addition, the elevation offsets changed from $0.075 \mathrm{~mm}$ to $0.6 \mathrm{~mm}$ instead of $0.1 \mathrm{~mm}$ to $0.8 \mathrm{~mm}$.

For the visualization, the computation time was one of the measurement criteria for the volume reconstruction algorithm as listed in Table 5-4. For the chicken, it took $1092932 \mathrm{~ms}$ or 18.22 minutes with 651 frames. The computation time for the volume reconstruction was 1.67 seconds per frame by $1092932 / 651$ [ms/frame]=1092.932/651 s/frames]. For the beef, it took $1092756 \mathrm{~ms}$ or 18.21 minutes with 651 frames. The computation time for the volume reconstruction was 1.67 seconds per frame.

Table 5-4: Computation time for the chicken/beef data set

\begin{tabular}{|l|l|l|}
\hline Phantom & Chicken [s/frames] & Beef [s/frames] \\
\hline $\begin{array}{l}\text { Computation Time for Volume } \\
\text { Reconstruction }\end{array}$ & 1.67 & 1.67 \\
\hline
\end{tabular}

The computation time was subject to the number of frames to process, the size of each image, computer systems environment, and operating system. In this thesis, executions occurred only in a single thread and single process with 651 frames with the image size of $400 \times 395$. It has thus not been optimized for the parallel programming. The system specification requirement was listed in Appendix A.

When comparing another algorithm by implementing Pixel Based Method (PBM) with an ellipsoid kernel and Gaussian weighting, Solberg et al. [18] noted that the execution time was 1.5 seconds per frame as listed in Table 5-5. The PBM method was faster than our implementation due to the smaller input image size and different system setup. The 
first led to less processing and displaying time. The second contributed the variations in the measurement.

Table 5-5: Comparisons between different reconstruction algorithms

\begin{tabular}{|l|l|l|l|}
\hline & $\begin{array}{l}\text { Input Images } \\
\text { Resolution } \\
{[\text { Pixels }]}\end{array}$ & Hardware System/OS & $\begin{array}{l}\text { Computation Time } \\
{[\mathrm{s} / \text { frame }]}\end{array}$ \\
\hline $\begin{array}{l}\text { Low resolution } \\
\text { algorithm }\end{array}$ & $400 \times 395$ & Core i7-2600, Windows 7 & 1.67 \\
\hline $\begin{array}{l}\text { PBM, Ellipsoid } \\
\text { kernel, Gaussian } \\
\text { weighting }\end{array}$ & $128 \times 128$ & $\begin{array}{l}\text { IBM RS6000, } \\
560 \text { workstation, } \\
\text { UNIX }\end{array}$ & 1.5 \\
\hline
\end{tabular}




\section{Chapter 6: Conclusion and Future Research}

\subsection{Conclusion}

There were three principal areas of study for this thesis:

In the first area of study, the improved procedure as developed in this thesis offered two contributions during the data acquisition process: a reduction of the spatial calibration time, and reduction of the overall acquisition time. These reduced time costs during the operation were crucial in maintaining consistency during the data acquisition process in phantoms. In addition, the wait time for sonographers and patients were reduced as well, improving the practical usability of US in clinical settings.

In summary, the data acquisition process started with the EM sensor attached to the ultrasound probe which scanned the spatial phantom with both the temporal spatial calibrations and the speckle phantom with the temporal calibration. Next, meat phantoms composed of beef and chicken were scanned with the temporal calibration. Therefore, input data including orientations, positions, and images were collected in series.

The second area of study involved data analysis. The fundamental building block was the speckle decorrelation method for considering both the fully developed speckle (FDS) and non-FDS regions.

Initially, the distance estimation took place within the in-plane motion where the images were divided into patches and the pixel intensities were converted into a linear scale. This was done after having completed the data acquisition when an algorithm selected a specific frame with respect to the elevation distance. The next step involved the calculation of the correlation coefficients between the patches in the images located 
for the out-of-plane motion. The transducer's specific decorrelation curve was obtained together with its axial, lateral, and elevation direction in the speckle phantom. The axial and lateral decorrelation curves were obtained from the test phantoms to better understand the effect of the coherent scattering.

An important contribution this thesis was in the reduction of the standard deviation during elevation distances estimations through searching the maximum correlation coefficients in the patches with respect to axial and lateral distances. By applying the improved algorithm under an isotropic medium, the adaptive FDS correlation curve was obtained for each patch in the beef and chicken phantoms' data sets where the amounts of coherent scattering existed. The estimated elevation distance could thus be found between frames from the given correlation coefficient. The improved method reduced the standard deviation by $5-10 \%$ when the elevation distance was situated between $0.15 \mathrm{~mm}$ and 0.375 $\mathrm{mm}$ in test phantoms. Therefore, the elevation distance accuracy was improved by reducing the marginal errors during the freehand ultrasound scans.

There were three reasons errors can occur. First, the average taken of many data points led into the variation that was found from the range of measurement in the specific correlation coefficients. Second, the elevation distance might lie outside the range of 0.15 $\mathrm{mm}$ to $0.375 \mathrm{~mm}$ in the steep region of the decorrelation curve. Finally, if the location of patches was deeper than what was outlined in this thesis, the inaccuracy increased due to the attenuation effect. If the patch was located closer to the surface, the accuracy increased.

The third area of study involved the $3 \mathrm{D}$ reconstruction algorithm from sequential 2D ultrasound images. Instead of displaying a high-resolution 3D voxel grid, it was advantageous to display a low-resolution voxel grid to reduce the amount of processing time necessary.

The algorithm presented in this thesis took 1.67 seconds per frame in computation time by using $400 \times 395$ pixels for the 3D volume reconstruction executing on a single thread and single process. While displaying the low-resolution volume in 3D, verifications were completed at the initial stage. 


\subsection{Future Research}

An important area of study worthy of continued pursuit in ultrasound technology pertains to the development of more sophisticated 3D reconstruction algorithm. Currently, the reconstruction algorithm lies on the center location and the intensity of patches in the images between the current frame and next frame. The accuracy of the reconstruction algorithm ought to be better considered when the probe is swept back and forth to increase the accuracy of the voxels' intensity. In addition, the reconstruction algorithm's cumulative errors that present themselves from one frame to the next need to be considered. Therefore, a more robust reconstruction algorithm is required.

The second area of future research relates to the optimization of the current 3D volume reconstruction algorithm. The current volume reconstruction takes about 1.67 seconds per image in trials involving test phantoms. The reconstruction algorithm can be further improved for the purpose of the real-time clinical application. The suggested solution is to implement a parallel processing in the graphics processor unit (GPU). 


\section{Appendices}

\section{Appendix A : External Library and System Environment}

\section{A.1 External Library}

There are two external libraries used in this thesis. They are listed below:

- OpenCV 3.0: The primary job for OpenCV library is to collect the images [103]. Be aware of that the newer version may be incompatible with the older version.

- Boost library 1.58.0: The primary job for Boost library is to deal with complicated matrix multiplication and windows file system [104].

- It is necessary to use tools such as MATLAB 2015b to plot graphs from data generated from our software application written in $\mathrm{C}++$.

\section{A.2 System Environment}

The operating system (OS) with CPU, RAM, and Independent Development Environment (IDE) are listed in the following.

- Operating System: Windows 7 64-bit

- CPU: Intel Core i7 2600K

- RAM: 12 GB of RAM

- IDE: Visual Studio 2013 supports C++11 compiler. 


\section{Appendix B Voxel File Format}

According to the slab6.txt within the zip file of slab6.zip, the kv6 file reading guide is listed as follows [101]. It has been done in the $\mathrm{C}$ programming language. If the implementation of importing kv6 is known, the exporting procedures can be realized through reverse engineering. The kv6 file format in slab6.txt is listed in the following: typedef struct \{ long col; unsigned short z; char vis, dir; \} kv6voxtype; long xsiz, ysiz, zsiz;

float xpiv, ypiv, zpiv; unsigned long xlen[xsiz]; unsigned short ylen[xsiz][ysiz]; long numvoxs; FILE * fil = fopen("?.KV6",rb"); fread(\&fileid,4,1,fil); //'Kvxl' (= 0x6c78764b in Little Endian) //Voxel grid dimensions fread(\&xsiz,4,1,fil); fread(\&ysiz,4,1,fil); fread(\&zsiz,4,1,fil); $/ /$ Pivot point. Floating point format. Voxel units. fread(\&xpiv,4,1,fil); fread(\&ypiv,4,1,fil); fread(\&zpiv,4,1,fil); fread(\&numvoxs, 4,1, fil); //Total number of surface voxels for $(\mathrm{i}=0 ; \mathrm{i}<$ numvoxs; $\mathrm{i}++) / / 8$ bytes per surface voxel, $Z$ 's must be sorted \{

red [i] = fgetc(fil);//Range: $0 . .255$

green[i] = fgetc(fil);//"

blue [i] = fgetc(fil);//"

dummy = fgetc(fil); //Always 128. Ignore.

height_low = fgetc(fil); //Z coordinate of this surface voxel

height_high = fgetc(fil);//"

visibility = fgetc(fil); //Low 6 bits say if neighbor is solid or air normalindex = fgetc(fil); //Uses 256-entry lookup table

\}

//Number of surface voxels present in plane $\mathrm{x}$ (extra information)

for $(\mathrm{x}=0 ; \mathrm{x}<\mathrm{xsiz} ; \mathrm{x}++)$ fread $(\& \mathrm{xlen}[\mathrm{x}], 4,1, \mathrm{fil})$;

$/ /$ Number of surface voxels present in column $(\mathrm{x}, \mathrm{y})$

for $(\mathrm{x}=0 ; \mathrm{x}<\mathrm{xsiz} ; \mathrm{x}++)$ for $(\mathrm{y}=0 ; \mathrm{y}<\mathrm{ysiz} ; \mathrm{y}++)$ fread (\&ylen[x][y],2,1,fil);

//Added 06/30/2007: suggested palette (optional)

fread(\&suggpalid,4,1,fil); //'SPal' (= 0x6C615053 in Little Endian)

fread(suggestedpalette,768,1,fil); //R,G,B,R,G,.. Value range: 0-63

fclose(fil) 


\section{Appendix C Procedures for making the Spatial Phantom}

Required materials are two nylon wires $1 \mathrm{~mm}$ in diameter and $1 \mathrm{~cm}$ in length; 400 $\mathrm{ml}$ of water, and $8 \mathrm{~g}$ of agar powder. The preparation process is listed below:

1. Read the material safety data sheet (MSDS) of agar powder from the supplier before doing the experiment [105].

2. Boil the water and pour it into a $100 \mathrm{ml}$ graduated cylinder.

3. Pour the graduated cylinder into $500 \mathrm{ml}$ beakers. It may require multiple attempts until the beaker is full of $400 \mathrm{ml}$ of hot water.

4. Pour the beakers into bows.

5. Stir for 1.5 hours so it becomes sticky. After it becomes sticky, place the two nylon wires at the bottom center of the bow and cross them together.

\section{Appendix D Procedures for making the Speckle Phantom}

Speckle phantom contains $400 \mathrm{ml}$ of water, $12 \mathrm{~g}$ of graphite powder, and $8 \mathrm{~g}$ of agar powder. Full safety protocol is followed for handling the graphite powder as unprotected exposure can lead to severe irritation in the eyes or respiratory tract and can burst into flames if handled improperly [106].

1. Read the material safety data sheet (MSDS) of graphite powder from the supplier before doing the experiment [106].

2. According to the MSDS, protective gear includes goggle, gloves and protective cloth. They are required to be worn during the preparation of the speckle phantom

3. Boil the water and pour it into a $100 \mathrm{ml}$ graduated cylinder.

4. Pour the graduate cylinder into $500 \mathrm{ml}$ beakers. It may require multiple attempts until the beaker is full of $400 \mathrm{ml}$ of hot water.

5. Pour the beakers into bows.

6. Stir for 1.5 hours so it becomes sticky. After it becomes sticky, place $12 \mathrm{~g}$ of graphite powder into the bowl and keep stirring. 
7. After the graphite powder becomes well distributed, it is time to stop stirring. The speckle phantom will solidify.

\section{Appendix E Procedures for making the Meat Phantoms}

Meat phantoms are composed of chicken, beef, and eraser. Materials are $500 \mathrm{ml}$ of water with $10 \mathrm{~g}$ of agar powder. The amount of water utilized is dependent on the height of the containers as all materials must be fully submerged under water. An intermediate layer is formed to remove direct contact between materials and probe after the phantom solidifies. The dimension of eraser is $2 \mathrm{~cm}(\mathrm{~L}) \mathrm{x} 0.5 \mathrm{~cm}(\mathrm{~W})$. The dimension of beef is $0.8 \mathrm{~cm}(\mathrm{~W}) \times 1.3 \mathrm{~cm}(\mathrm{~L})$. The dimension of chicken is $0.8 \mathrm{~cm}(\mathrm{~W}) \times 1.3 \mathrm{~cm}(\mathrm{~L})$. The procedures for setting up the meat phantoms follow:

1. Read the material safety data sheet (MSDS) of agar powder from the supplier before doing the experiment [105].

2. Boil the water and pour it into a $100 \mathrm{ml}$ graduated cylinder.

3. Pour the graduate cylinder into $1000 \mathrm{ml}$ beakers. It may require multiple attempts for the beaker to be filled with $500 \mathrm{ml}$ of hot water.

4. Pour the beakers into a rectangular container such as a lunch box.

5. Stir for 1.5 hours until it becomes sticky. After it becomes sticky, place the chicken, beef, and eraser into the rectangular box.

\section{Appendix F Procedures for Setting up SonixTablet Ultrasound System}

1. Select on "Menu" bottom->"Administrator" bottom->"Capture" bottom

2. Click on "Cine Advanced"

a. In "Compressor", select "Non-compression"

b. Click "Prospective".

3. Click "OK" bottom.

By selecting the "Non-Compression", the system stores the video clips as .avi without compression. In addition, selecting the "Prospective" option means that the recording starts after pressing "1" on touch screen monitor. 


\section{References}

[1] H. Wackerhage and M. J. Rennie, "How nutrition and exercise maintain the human musculoskeletal mass," J. Anat., vol. 208, no. 4, pp. 451-458, 2006.

[2] M. Leunig, P. E. Beaulé, and R. Ganz, "The concept of femoroacetabular impingement: current status and future perspectives," Clin. Orthop. Relat. Res., vol. 467, no. 3, pp. 616-622, 2009.

[3] A. L. Tan, R. J. Wakefield, P. G. Conaghan, P. Emery, and D. McGonagle, "Imaging of the musculoskeletal system: magnetic resonance imaging, ultrasonography and computed tomography," Best Pract. Res. Clin. Rheumatol., vol. 17, no. 3, pp. 513-528, 2003.

[4] S. Lerch, A. Kasperczyk, J. Warnecke, T. Berndt, and O. Rühmann, "Evaluation of Cam-type femoroacetabular impingement by ultrasound," Int. Orthop., vol. 37, no. 5, pp. 783-788, May 2013.

[5] T. R. Nelson and T. T. Elvins, "Visualization of 3D ultrasound data," IEEE Comput. Graph. Appl., vol. 13, no. 6, pp. 50-57, 1993.

[6] R. J. Wakefield et al., "The value of sonography in the detection of bone erosions in patients with rheumatoid arthritis: a comparison with conventional radiography," Arthritis Rheumatol., vol. 43, no. 12, pp. 2762-2770, 2000.

[7] A. Rouhandeh, "Non-invasive Assessment and Compensation of Soft-tissue Artefacts in Hip Joint Kinematics," Carleton University, 2014.

[8] A. Fenster, K. J. M. Surry, G. R. Mills, and D. B. Downey, "3D ultrasound guided breast biopsy system," in Ultrasonics, 2004, vol. 42, no. 1-9, pp. 769-774.

[9] K. Karadayi, Y. Kim, R. Managuli, and R. Managuli, “Three-Dimensional Ultrasound: From Acquisition to Visualization and from Algorithms to Systems," IEEE Rev. Biomed. Eng., vol. 2, pp. 23-39, 2009.

[10] A. Gee, R. Prager, G. Treece, and L. Berman, "Engineering a freehand 3D ultrasound system," Pattern Recognit. Lett., vol. 24, no. 4-5, pp. 757-777, 2003.

[11] P. W. Hsu, R. W. Prager, a H. Gee, and G. M. Treece, "Real-time freehand 3D ultrasound calibration," Ultrasound Med Biol, vol. 34, no. September, pp. 239251, 2008.

[12] P. W. Hsu, R. W. Prager, A. H. Gee, and G. M. Treece, "Freehand 3D ultrasound calibration: A review," in Advanced Imaging in Biology and Medicine: Technology, Software Environments, Applications, 2009, pp. 47-84.

[13] R. 0 Bude and R. S. Adler, "An Easily Made, Low-Cost, Tissue-Like Ultrasound Phantom Material," J. Clin. Ultrasound, vol. 23, no. May, pp. 271-273, 1995.

[14] K. Kim, C. G. Jeong, and S. J. Hollister, "Non-invasive monitoring of tissue scaffold degradation using ultrasound elasticity imaging," Acta Biomater., vol. 4, no. 4, pp. 783-790, 2008.

[15] H. Gao, Q. Huang, X. Xu, and X. Li, "Wireless and sensorless 3D ultrasound imaging," Neurocomputing, vol. 195, pp. 159-171, 2016.

[16] A. H. Gee, R. James Housden, P. Hassenpflug, G. M. Treece, and R. W. Prager, "Sensorless freehand 3D ultrasound in real tissue: Speckle decorrelation without fully developed speckle," Med. Image Anal., vol. 10, no. 2, pp. 137-149, 2006.

[17] K. Hajian-Tilaki, "Sample size estimation in diagnostic test studies of biomedical informatics," J. Biomed. Inform., vol. 48, pp. 193-204, 2014. 
[18] O. V. Solberg, F. Lindseth, H. Torp, R. E. Blake, and T. A. N. Hernes, "Freehand 3D ultrasound reconstruction algorithms - a review," Ultrasound Med. Biol., vol. 33, no. 7, pp. 991-1009, 2007.

[19] J. M. Sanches and J. S. Marques, "A multiscale algorithm for three-dimensional free-hand ultrasound," Ultrasound Med. Biol., vol. 28, no. 8, pp. 1029-1040, 2002.

[20] T. A. Tuthill, J. F. Krücker, J. B. Fowlkes, and P. L. Carson, "Automated threedimensional US frame positioning computed from elevational speckle decorrelation.," Radiology, vol. 209, no. 2, pp. 575-582, 1998.

[21] J. I. Silver, "Development of ultrasound based techniques for measuring skeletal muscle motion," Diss. Carlet. Univ. Ottawa, 2009.

[22] T. O'Shea, J. Bamber, D. Fontanarosa, S. van der Meer, F. Verhaegen, and E. Harris, "Review of ultrasound image guidance in external beam radiotherapy part II: intra-fraction motion management and novel applications," Phys. Med. Biol., vol. 61, no. 8, p. R90, 2016.

[23] T. Szabó, Diagnostic ultrasound imaging : inside out. Amsterdam Boston: Elsevier Academic, 2004.

[24] P. Hoskins, K. Martin, and A. Thrush, Diagnostic ultrasound: physics and equipment. Cambridge, UK New York: Cambridge University Press, 2010.

[25] S. Bushong and B. Archer, Diagnostic ultrasound: Physics, biology, and instrumentation. Mosby-Year Book, 1991.

[26] F. Kremkau, Diagnostic ultrasound: principles and instruments Seventh ed. St. Louis, Mo: Saunders Elsevier, 2006.

[27] M. A. Bahramabadi, "Sensorless Out-of-Plane Displacement Estimation for Freehand 3D Ultrasound Applications," Carleton University, 2014.

[28] W. R. Hedrick, D. L. Hykes, and D. E. Starchman, Ultrasound physics and instrumentation. St. Louis, Mo: Elsevier Mosby, 2005.

[29] J. Prince and J. M. Links, Medical imaging signals and systems. Upper Saddle River, N.J: Pearson Prentice Hall, 2006.

[30] R. L. Lieber and J. Friden, "Functional and clinical significance of skeletal muscle architecture," Muscle Nerve, vol. 23, pp. 1647-1666, 2000.

[31] M. Rana and J. M. Wakeling, "In-vivo determination of 3D muscle architecture of human muscle using free hand ultrasound," J. Biomech., vol. 44, no. 11, pp. 2129 2135, 2011.

[32] S. Elwagdy, S. Ghoneim, S. Moussa, and I. Ewis, "Three-dimensional ultrasound (3D US) methods in the evaluation of calcular and non-calcular ureteric obstructive uropathy," World J. Urol., vol. 26, no. 3, pp. 263-274, 2008.

[33] G. Carneiro, F. Amat, B. Georgescu, S. Good, and D. Comaniciu, "Semantic-based indexing of fetal anatomies from 3-D ultrasound data using global/semi-local context and sequential sampling," in 26th IEEE Conference on Computer Vision and Pattern Recognition, CVPR, 2008.

[34] R. L. Dyson et al., "Three-dimensional ultrasound in the evaluation of fetal anomalies," in Ultrasound in Obstetrics and Gynecology, 2000, vol. 16, no. 4, pp. $321-328$.

[35] G. L. Fontaine, D. H. Rodgerson, R. R. Hanson, and R. Steiger, "Ultrasound Evaluation of Equine Gastrointestinal Disorders," Compend. Equine Ed., vol. 21, no. 3, 1999. 
[36] A. Fenster and D. B. Downey, "3-D ultrasound imaging: A review," IEEE Eng. Med. Biol. Mag., vol. 15, no. 6, pp. 41-51, 1996.

[37] A. Fenster, D. B. Downey, and H. N. Cardinal, "Three-dimensional ultrasound imaging.," Phys. Med. Biol., vol. 46, no. 5, pp. R67-R99, 2001.

[38] L. Nock, G. E. Trahey, and S. W. Smith, "Phase aberration correction in medical ultrasound using speckle brightness as a quality factor," J. Acoust. Soc. Am., vol. 85, no. 5, pp. 1819-1833, 1989.

[39] J. M. Thijssen, "Ultrasonic speckle formation, analysis and processing applied to tissue characterization," Pattern Recognit. Lett., vol. 24, no. 4, pp. 659-675, 2003.

[40] N. Afsham, M. Najafi, P. Abolmaesumi, and R. Rohling, "Out-of-plane motion estimation based on a Rician-Inverse Gaussian model of RF ultrasound signals: speckle tracking without fully developed speckle," Proc. SPIE Med. Imaging Ultrason. Imaging, Tomogr. Ther., vol. 8320, pp. 832017-832017-7, 2012.

[41] N. Afsham, M. Najafi, P. Abolmaesumi, and R. Rohling, "A generalized correlation-based model for out-of-plane motion estimation in freehand ultrasound," IEEE Trans. Med. Imaging, vol. 33, no. 1, pp. 186-199, 2014.

[42] M. F. Insana, R. F. Wagner, R. Momenan, and G. G. Cox, "Quantitative Ultrasonic Imaging," Proc. SPIE, vol. 914. pp. 32-39, 1988.

[43] J. A. Jensen, "A model for the propagation and scattering of ultrasound in tissue," J. Acoust. Soc. Am., vol. 89, no. 1, pp. 182-190, 1991.

[44] J. Ng, R. Prager, N. Kingsbury, G. Treece, and A. Gee, "Modeling ultrasound imaging as a linear, shift-variant system," ieee Trans. Ultrason. Ferroelectr. Freq. Control, vol. 53, no. 3, pp. 549-563, 2006.

[45] J. W. Goodman, "Statistical properties of laser speckle patterns," Laser Speckle Relat. Phenom., vol. 6, pp. 9-75, 1975.

[46] R. F. Wagner, S. W. Smith, J. M. Sandrik, and H. Lopez, "Statistics of speckle in ultrasound B-scans," IEEE Trans. sonics Ultrason., vol. 30, no. 3, pp. 156-163, 1983.

[47] R. W. Prager, A. H. Gee, G. M. Treece, C. J. C. Cash, and L. H. Berman, "Sensorless freehand 3-D ultrasound using regression of the echo intensity," Ultrasound Med. Biol., vol. 29, no. 3, pp. 437-446, 2003.

[48] J.-F. Chen, J. B. Fowlkes, P. L. Carson, and J. M. Rubin, "Determination of scanplane motion using speckle decorrelation: Theoretical considerations and initial test," Int J Imaging Sys Technol, vol. 8, no. 1, pp. 38-44, 1997.

[49] R. W. Prager, U. Z. Ijaz, A. H. Gee, and G. M. Treece, "Three-dimensional ultrasound imaging," Proc. Inst. Mech. Eng. Part H J. Eng. Med., vol. 224, no. 2, pp. 193-223, 2010.

[50] R. N. Rohling, "3D freehand ultrasound: reconstruction and spatial compounding," University of Cambridge, 1999.

[51] D. E. O. Dewi, M. M. Fadzil, A. A. M. Faudzi, E. Supriyanto, and K. W. Lai, "Position Tracking Systems for Ultrasound Imaging: A Survey," in Medical Imaging Technology, M. A. Haidekker, Ed. Springer Singapore, 2015, pp. 57-89.

[52] F. H. Raab, E. B. Blood, T. O. Steiner, and H. R. Jones, "Magnetic position and orientation tracking system," IEEE Trans. Aerosp. Electron. Syst., no. 5, pp. 709$718,1979$.

[53] W. W. Gibbon, G. Long, D. A. Barron, and P. J. O'Connor, “Complications of 
orthopedic implants: Sonographic evaluation," J. Clin. Ultrasound, vol. 30, no. 5, pp. 288-299, 2002.

[54] R. W. Prager, A. Gee, and L. Berman, "Stradx: real-time acquisition and visualization of freehand three-dimensional ultrasound," Med. Image Anal., vol. 3, no. 2, pp. 129-140, 1999.

[55] P.-W. Hsu, "Freehand Three-Dimensional Ultrasound Calibration," University of Cambridge, 2008.

[56] L. Mercier, T. Langø, F. Lindseth, and L. D. Collins, "A review of calibration techniques for freehand 3-D ultrasound systems," Ultrasound Med. Biol., vol. 31, no. 2, pp. 143-165, 2005.

[57] R. W. Prager, R. N. Rohling, A. H. Gee, and L. Berman, "Rapid calibration for 3D freehand ultrasound," Ultrasound Med. Biol., vol. 24, no. 6, pp. 855-869, 1998.

[58] N. Pagoulatos, D. R. Haynor, and Y. Kim, "A fast calibration method for 3-D tracking of ultrasound images using a spatial localizer," Ultrasound Med. Biol., vol. 27, no. 9, pp. 1219-1229, 2001.

[59] R. Rohling, A. Gee, and L. Berman, "A comparison of freehand three-dimensional ultrasound reconstruction techniques," Med. Image Anal., vol. 3, no. 4, pp. 339359, 1999.

[60] P. Coupé, P. Hellier, X. Morandi, and C. Barillot, "Probe trajectory interpolation for 3D reconstruction of freehand ultrasound," Med. Image Anal., vol. 11, no. 6, pp. 604-615, 2007.

[61] R. Prager, A. Gee, G. Treece, and L. Berman, "Freehand 3D ultrasound without voxels: volume measurement and visualisation using the Stradx system," Ultrasonics, vol. 40, no. 1, pp. 109-115, 2002.

[62] R. R. Entrekin, B. A. Porter, H. H. Sillesen, A. D. Wong, P. L. Cooperberg, and C. H. Fix, "Real-time spatial compound imaging: Application to breast, vascular, and musculoskeletal ultrasound," Seminars in Ultrasound CT and MRI, vol. 22, no. 1. pp. 50-64, 2001.

[63] J. W. Trobaugh, D. J. Trobaugh, and W. D. Richard, "Three-dimensional imaging with stereotactic ultrasonography," Comput. Med. Imaging Graph., vol. 18, no. 5, pp. 315-323, 1994.

[64] N. Thune, O. H. Gilja, T. Hausken, and K. Matre, "A practical method for estimating enclosed volumes using 3D ultrasound," Eur. J. ultrasound, vol. 3, no. 1, pp. 83-92, 1996.

[65] S. Berg et al., "Dynamic three-dimensional freehand echocardiography using raw digital ultrasound data," Ultrasound Med. Biol., vol. 25, no. 5, pp. 745-753, 1999.

[66] C. D. Barry et al., "Three-dimensional freehand ultrasound: image reconstruction and volume analysis," Ultrasound Med. Biol., vol. 23, no. 8, pp. 1209-1224, 1997.

[67] Q. H. Huang, Y. P. Zheng, M. H. Lu, and Z. R. Chi, "Development of a portable 3D ultrasound imaging system for musculoskeletal tissues," Ultrasonics, vol. 43, no. 3, pp. 153-163, 2005.

[68] Q. H. Huang, Z. Yang, W. Hu, L. W. Jin, G. Wei, and X. Li, "Linear Tracking for 3-D Medical Ultrasound Imaging," IEEE Trans. Cybern., vol. 43, no. 6, pp. 17471754, 2013.

[69] X. Chen et al., "Reconstruction of freehand 3D ultrasound based on kernel regression," Biomed. Eng. Online, vol. 13, no. 1, p. 124, 2014. 
[70] F. Hottier and A. C. Billon, "3D Ecbograpby: Status and Perspective," 1990.

[71] H. A. McCann, J. C. Sharp, T. M. Kinter, C. N. McEwan, C. Barillot, and J. F. Greenleaf, "Multidimensional ultrasonic imaging for cardiology," Proc. IEEE, vol. 76, no. 9, pp. 1063-1073, 1988.

[72] R. S. J. Estépar, M. Martin-Fernandez, C. Alberola-Lopez, J. Ellsmere, R. Kikinis, and C.-F. Westin, "Freehand ultrasound reconstruction based on ROI prior modeling and normalized convolution," in International Conference on Medical Image Computing and Computer-Assisted Intervention, 2003, pp. 382-390.

[73] R. San José-Estépar, M. MARTIn-FERNAndez, P. P. Caballero-Mart'linez, C. Alberola-López, and J. Ruiz-Alzola, "A theoretical framework to threedimensional ultrasound reconstruction from irregularly sampled data," Ultrasound Med. Biol., vol. 29, no. 2, pp. 255-269, 2003.

[74] J. M. Sanches and J. S. Marques, "A Rayleigh reconstruction/interpolation algorithm for 3D ultrasound," Pattern Recognit. Lett., vol. 21, no. 10, pp. 917-926, 2000.

[75] C. Charbonnier, L. Assassi, P. Volino, and N. Magnenat-Thalmann, "Motion study of the hip joint in extreme postures," Vis. Comput., vol. 25, no. 9, pp. 873-882, 2009.

[76] F. Lindseth, T. Langø, J. Bang, N. Hernes, and A. Toril, "Accuracy evaluation of a 3D ultrasound-based neuronavigation system," Comput. Aided Surg., vol. 7, no. 4, pp. 197-222, 2002.

[77] G. M. Treece, R. W. Prager, A. H. Gee, and L. Berman, "Correction of probe pressure artifacts in freehand 3D ultrasound," Med. Image Anal., vol. 6, no. 3, pp. 199-214, 2002.

[78] D. G. Gobbi and T. M. Peters, "Interactive Intra-operative 3D Ultrasound Reconstruction and Visualization," in International Conference on Medical Image Computing and Computer-Assisted Intervention, D. Hutchison and J. C. Mitchell, Eds. 2002, pp. 156-163.

[79] D. G. Gobbi, B. K. Lee, and T. M. Peters, "Correlation of pre-operative MRI and intra-operative 3D ultrasound to measure brain tissue shift," Proc. SPIE, vol. 3982, no. 2001, pp. 77-84, 2000.

[80] A. Gronningsaeter, A. Kleven, S. Ommedal, T. E. Aarseth, T. Lie, and F. Lindseth, "SonoWand, an ultrasound-based neuronavigation system," Neurosurgery, vol. 47, no. 6, pp. 1373-1380, 2000.

[81] Å. Birkeland et al., "The ultrasound visualization pipeline," Math. Vis., vol. 37, pp. 283-303, 2014.

[82] T. R. Nelson and D. H. Pretorius, "Interactive acquisition, analysis, and visualization of sonographic volume data," Int. J. Imaging Syst. Technol., vol. 8, no. 1, pp. 26-37, 1997.

[83] M. D. Ralston and R. M. Coleman, "Practical Imaging Informatics: Foundations and Applications for PACS Professionals" i $\{$ \} \} inde," Introd. to PACS, Bart. F. Branstetter IV (Ed.), Springer, New York, pp. 33-49, 2009.

[84] T. R. Nelson and D. H. Pretorius, "Three-dimensional ultrasound imaging," Ultrasound Med. Biol., vol. 24, no. 9, pp. 1243-1270, 1998.

[85] T. Gorkin, "Volume Rendering using Graphics Hardware," Direct, 2009. .

[86] D. Weiskopf, GPU-based interactive visualization techniques. Springer Science \& 
Business Media, 2006.

[87] A. Glassner, An Introduction to Ray Tracing, vol. 8. 1989.

[88] M. Belohlavek, V. Dutt, J. F. Greenleaf, D. A. Foley, T. C. Gerber, and J. B. Seward, "Multidimensional ultrasonic visualization in cardiology," in Ultrasonics Symposium, 1992. Proceedings., IEEE 1992, 1992, pp. 1137-1145 vol.2.

[89] Y. Sato et al., "Image guidance of breast cancer surgery using 3-D ultrasound images and augmented reality visualization," IEEE Trans. Med. Imaging, vol. 17, no. 5, pp. 681-693, 1998.

[90] R. J. Housden, A. H. Gee, G. M. Treece, and R. W. Prager, "Subsample interpolation strategies for sensorless freehand 3D ultrasound," Ultrasound Med. Biol., vol. 32, no. 12, pp. 1897-1904, 2006.

[91] R. J. Housden, A. H. Gee, G. M. Treece, and R. W. Prager, "Sensorless reconstruction of unconstrained freehand 3D ultrasound data," Ultrasound Med. Biol., vol. 33, no. 3, pp. 408-419, 2007.

[92] H. Rivaz, E. Boctor, and G. Fichtinger, "A robust meshing and calibration approach for sensorless freehand 3d ultrasound," Int. Soc. Opt. Eng. Med. Imaging, vol. 6513, p. 651318 1--8, 2007.

[93] P. Hassenpflug, R. W. Prager, G. M. Treece, and A. H. Gee, "Speckle classification for sensorless freehand 3-D ultrasound," Ultrasound Med. Biol., vol. 31, no. 11, pp. 1499-1508, 2005.

[94] C. Laporte and T. Arbel, "Learning to estimate out-of-plane motion in ultrasound imagery of real tissue," Med. Image Anal., vol. 15, no. 2, pp. 202-213, 2011.

[95] Ultrasonix Medical Corporation, “00.040.711.EN SonixTablet Extended User Manual Rev01 141029,” 2013. [Online]. Available:

http://www.bkultrasound.com/support/ultrasonix/user-manuals. [Accessed: 10-Jan2017].

[96] Polhemus, "POLHEMUS INNOVATION IN MOTION,” 2017. [Online]. Available: http://www.polhemus.com/motion-tracking/all-trackers/patriot/. [Accessed: 01-Aug-2016].

[97] "WikiSonix," 2015. [Online]. Available: http://www.ultrasonix.com/wikisonix/index.php/Main_Page. [Accessed: 01-Aug2016].

[98] Polhemus, "PATRIOT USER MANUAL," Colchester, Vermont, U.S.A, 2013.

[99] W. L. Smith and A. Fenster, "Optimum scan spacing for three-dimensional ultrasound by speckle statistics," Ultrasound Med. Biol., vol. 26, no. 4, pp. 551$562,2000$.

[100] J. Conrath and C. Laporte, "Towards improving the accuracy of sensorless freehand 3D ultrasound by learning," in Lecture Notes in Computer Science (including subseries Lecture Notes in Artificial Intelligence and Lecture Notes in Bioinformatics), 2012, vol. 7588 LNCS, pp. 78-85.

[101] K. Silverman, "Slab6 editor," 2003. [Online]. Available: http://www.advsys.net/ken/slab6.zip. [Accessed: 01-Aug-2016].

[102] K. Silverman, "Voxlap engine," 2003. [Online]. Available: http://advsys.net/ken/voxlap.htm. [Accessed: 01-Aug-2016].

[103] OpenCV, "OpenCV on-line documentation," 2015. [Online]. Available: http://docs.opencv.org/3.0.0/. [Accessed: 01-Aug-2016]. 
[104] B. Dawes, E. Niebler, R. Rivera, D. James, and V. Prus, “Boost 1.58.0 Library Documentation," 2015. [Online]. Available:

http://www.boost.org/users/history/version_1_58_0.html. [Accessed: 07-Jan2017].

[105] BioShop Canada Inc, "MATERIAL SAFETY DATA SHEET AGAR,” 2011. [Online]. Available: http://www.bioshopcanada.com/msds/AGR001.pdf. [Accessed: 01-Aug-2016].

[106] A. Alfa, "Material Safety Data Sheet Graphite Powder," Thermal Fisher Scientific Chemicals Inc, 2012. [Online]. Available:

https://www.alfa.com/en/content/msds/USA/10129.pdf. [Accessed: 01-Aug-2016]. 